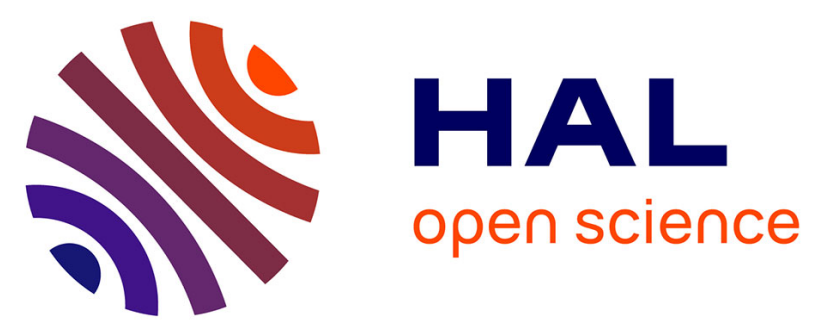

\title{
Les séquences loessiques pléistocène supérieur d'Havrincourt (Pas-de-Calais, France) : stratigraphie, paléoenvironnements, géochronologie et occupations paléolithiques
}

Pierre Antoine, Emilie Goval, Guillaume Jamet, Sylvie Coutard, Olivier Moine, David Hérisson, Patrick Auguste, Gilles Guérin, France Lagroix, Esther Schmidt, et al.

\section{- To cite this version:}

Pierre Antoine, Emilie Goval, Guillaume Jamet, Sylvie Coutard, Olivier Moine, et al.. Les séquences loessiques pléistocène supérieur d'Havrincourt (Pas-de-Calais, France): stratigraphie, paléoenvironnements, géochronologie et occupations paléolithiques. Quaternaire, 2014, 25 (4), pp.321-368. 10.4000/quaternaire.7278 . hal-01483554

\author{
HAL Id: hal-01483554 \\ https://hal.science/hal-01483554
}

Submitted on 4 Sep 2020

HAL is a multi-disciplinary open access archive for the deposit and dissemination of scientific research documents, whether they are published or not. The documents may come from teaching and research institutions in France or abroad, or from public or private research centers.
L'archive ouverte pluridisciplinaire HAL, est destinée au dépôt et à la diffusion de documents scientifiques de niveau recherche, publiés ou non, émanant des établissements d'enseignement et de recherche français ou étrangers, des laboratoires publics ou privés. 


\section{Quaternaire}

Revue de l'Association française pour l'étude du

Quaternaire

vol. 25/4 | 2014

Volume 25 Numéro 4

\section{Les séquences loessiques pléistocène supérieur} d'Havrincourt (Pas-de-Calais, France) : stratigraphie, paléoenvironnements, géochronologie et occupations paléolithiques

The upper pleistocene loess sequences of Havrincourt (Pas-de-Calais, France): stratigraphy, palaeoenvironments, geochronology and human occupations

Pierre Antoine, Emilie Goval, Guillaume Jamet, Sylvie Coutard, Olivier Moine, David Hérisson, Patrick Auguste, Gilles Guérin, France Lagroix, Esther Schmidt, Vincent Robert, Nick Debenham, Sascha Meszner et JeanJacques Bahain

\section{OpenEdition}

Journals

Édition électronique

URL : http://journals.openedition.org/quaternaire/7278

DOI : 10.4000 /quaternaire.7278

ISSN : $1965-0795$

Éditeur

Association française pour l'étude du quaternaire

Édition imprimée

Date de publication : 1 décembre 2014

Pagination : 321-368

ISSN : 1142-2904

Référence électronique

Pierre Antoine, Emilie Goval, Guillaume Jamet, Sylvie Coutard, Olivier Moine, David Hérisson, Patrick Auguste, Gilles Guérin, France Lagroix, Esther Schmidt, Vincent Robert, Nick Debenham, Sascha Meszner et Jean-Jacques Bahain, «Les séquences loessiques pléistocène supérieur d'Havrincourt (Pas-de-Calais, France) : stratigraphie, paléoenvironnements, géochronologie et occupations paléolithiques », Quaternaire [En ligne], vol. 25/4 | 2014, mis en ligne le 01 décembre 2016, consulté le 16 décembre 2019. URL : http://journals.openedition.org/quaternaire/7278; DOI : 10.4000/ quaternaire.7278 


\title{
LES SÉQUENCES LOESSIQUES PLÉISTOCÈNE SUPÉRIEUR D'HAVRINCOURT (PAS-DE-CALAIS, FRANCE) : STRATIGRAPHIE, PALÉOENVIRONNEMENTS, GÉOCHRONOLOGIE ET OCCUPATIONS PALÉOLITHIQUES
}

\author{
Pierre ANTOINE ${ }^{1}$, Emilie GOVAL ${ }^{2,3}$, Guillaume JAMET ${ }^{4}$, Sylvie COUTARD ${ }^{1,2}$, \\ Olivier MOINE ${ }^{1}$, David HÉRISSON ${ }^{3,5}$, Patrick AUGUSTE ${ }^{6}$, Gilles GUÉRIN ${ }^{7}$, \\ France LAGROIX ${ }^{8}$, Esther SCHMIDT ${ }^{7}$, Vincent ROBERT ${ }^{1}$, Nick DEBENHAM ${ }^{9}$, \\ Sascha MESZNER ${ }^{10} \&$ Jean-Jacques BAHAIN ${ }^{3}$
}

\section{RÉSUMÉ}

\begin{abstract}
Dans le cadre des travaux préalables au creusement du futur Canal Seine-Nord Europe (liaison Seine-Escaut), une opération de diagnostic archéologique de grande ampleur a été menée entre 2008 et 2011 dans le nord de la France. A Havrincourt (Pas-deCalais), la découverte de plusieurs niveaux de silex paléolithiques inclus dans une séquence loessique relativement épaisse $(\sim 6-7 \mathrm{~m})$ préservée sur un versant en pente douce exposé nord-est a débouché sur une opération de fouille sur une surface totale de $6000 \mathrm{~m}^{2}$. Quatre niveaux paléolithiques en position primaire ont été mis au jour. Cette séquence a fait l'objet d'un travail de suivi stratigraphique en continu sur plus de neuf mois et d'une approche interdisciplinaire associant pédostratigraphie, sédimentologie, micromorphologie, paléontologie, malacologie, magnétisme environnemental, datations OSL et ${ }^{14} \mathrm{C}$. Sur la base de ces recherches, nous proposons : 1) une nouvelle séquence pédostratigraphique de référence pour le nord de la France, notamment en ce qui concerne le début du Pléniglaciaire inférieur et le Pléniglaciaire moyen ( 70-30 ka), 2) un nouveau canevas géochronologique régional pour le Dernier Glaciaire (datations par luminescence et radiocarbone) ainsi qu'une révision du schéma pédostratigraphique et chronoclimatique régional, et 3) la description et la datation d'une succession unique d'horizons périglaciaires comportant cinq réseaux de fentes associés à des gleys de toundra qui constitue une première séquence régionale de référence. Par ailleurs, la bonne conservation des niveaux archéologiques, associée à l'analyse pluridisciplinaire de la séquence pédosédimentaire et aux datations, débouche sur le calage chronostratigraphique et chrono-climatique précis des quatre niveaux paléolithiques fouillés incluant un ensemble exceptionnel attribuable au Paléolithique supérieur ancien (Gravettien ancien). Ces résultats et découvertes font d'Havrincourt une nouvelle séquence pédostratigraphique et archéologique de référence pour le Pléistocène supérieur de la France septentrionale et plus largement pour la zone loessique ouest-européenne.
\end{abstract}

Mots-clés : loess, paléosols, Pléistocène supérieur, nord de la France, structures périglaciaires, paléoenvironnement, Paléolithique

\footnotetext{
${ }^{1}$ Laboratoire de Géographie Physique, Environnements quaternaires et actuels (UMR 8591, CNRS-Université Paris 1-UPEC), 1 place Aristide Briand, FR-92195 MEUDON cedex. Courriels : pierre.antoine@cnrs-bellevue.fr, olivier.moine@cnrs-bellevue.fr, vincent.robert@cnrs-bellevue.fr

${ }^{2}$ Institut National de Recherches Archéologiques Préventives, Nord Picardie, 518 rue Saint-Fuscien, FR-80 000 AMIENS.

Courriels : emilie.goval@inrap.fr, sylvie.coutard@inrap.fr

${ }^{3}$ Muséum national d'Histoire naturelle, Département de Préhistoire (UMR 7194 CNRS-Institut de Paléontologie Humaine), 1 rue René Panhard, FR-75013 PARIS. Courriel : bahain@mnhn.fr

${ }^{4}$ Laboratoire de Morphodynamique Continentale et Côtière (UMR 6143 CNRS-Université de Caen, Campus 1), 24 rue des Tilleuls, FR-14 000 CAEN. Courriel : guillaume.jamet@unicaen.fr

${ }^{5}$ Institut National de Recherches Archéologiques Préventives, Canal Seine-Nord Europe, 16 rue du Général Leclerc, FR-80 600 CROIXMOLIGNEAUX. Courriel : david.herisson@inrap.fr

${ }^{6}$ Laboratoire Géosystèmes (UMR 8217 CNRS-Université de Lille 1), Bâtiment SN 5, FR-59 655 VILLENEUVE-D’ASCQ cedex.

Courriel : patrick.auguste@univ-lille1.fr

${ }^{7}$ Laboratoire des Sciences du Climat et de l'Environnement (LSCE/IPSL, CEA-CNRS-UVSQ), Domaine du CNRS, avenue de la Terrasse,

FR-91 198 GIF-SUR-YVETTE. Courriels : gilles.guerin@1sce.ipsl.fr

${ }^{8}$ Institut de Physique du Globe de Paris (IPGP), 1 rue Jussieu, FR-75 238 PARIS. Courriel : lagroix@ipgp.fr

${ }^{9}$ Quaternary TL surveys, 19 Leonard avenue, Nottingham, NG5-2LW, UNITED KINGDOM. Courriel : n.debenham@qtls.globalnet.co.uk.

${ }^{10}$ Lehrstuhl Physische Geographie, Institut für Geographie, TU Dresden, DE-01062 DRESDEN. Courriel : sascha.meszner@tu-dresden.de
} 
ABSTRACT

THE UPPER PLEISTOCENE LOESS SEQUENCES OF HAVRINCOURT (PAS-DE-CALAIS, FRANCE): STRATIGRAPHY, PALAEOENVIRONMENTS, GEOCHRONOLOGY AND HUMAN OCCUPATIONS

In connection with the future Canal Seine-Nord Europe (Seine-Scheldt), a large-scale rescue archaeological survey was conducted between 2008 and 2011 in northern France. At Havrincourt (Pas-de-Calais), the discovery of several levels of Palaeolithic flint artefacts embedded in a relatively thick loess sequence $(\sim 6-7 \mathrm{~m})$ preserved on a gentle slope facing north-east, has resulted in a rescue archaeological excavation on a total surface of $6000 \mathrm{~m}^{2}$. Four Palaeolithic levels were uncovered in primary position. This sequence has been the subject of a continuous stratigraphic survey over nine months and an interdisciplinary approach combining pedostratigraphy, sedimentology, micromorphology, palaeontology, malacology, environmental magnetism, OSL and ${ }^{14} \mathrm{C}$ dating. Based on this research, we propose: 1) a new pedostratigraphic reference sequence for Northern France, especially with regard to the Lower and Middle Pleniglacial periods ( 70-30 ka), 2) a new regional geochronological framework for the Last Glacial (luminescence and radiocarbon) as well as a revision of the regional pedostratigraphic and chrono-climatic systems, and 3) the description and dating of a unique succession of periglacial horizons including five ice-wedge cast networks associated with tundra gley horizons representing a first regional reference sequence. In addition, the good preservation of archaeological levels, associated with the multidisciplinary analysis and dating of the pedosedimentary sequences leads to the chronostratigraphic and chrono-climatic interpretation of the four Palaeolithic levels including a unique occupation related to the Early Upper Palaeolithic (Early Gravettian). These results and new discoveries allow us to propose Havrincourt as a new pedostratigraphic and archaeological reference sequence for the Late Pleistocene of northern France and more broadly for the west-European loess area.

Keywords: loess, palaeosols, Upper Pleistocene, north of France, periglacial features, palaeoenvironnement, Palaeolithic

\section{1 - INTRODUCTION}

Le gisement d'Havrincourt se situe dans la partie occidentale de la ceinture loessique européenne, dans le département du Pas-de-Calais (fig. 1A) à une quinzaine de kilomètres au sud-ouest de Cambrai et à proximité immédiate du Canal du Nord (fig. 1B). L'étude des séquences loessiques d'Havrincourt s'inscrit dans le projet du Canal Seine-Nord Europe destiné à relier les bassins de la Seine et de l'Escaut à travers le nord de la France. Dans le cadre des travaux préalables aux terrassements, des opérations de diagnostic archéologique de grande ampleur ont été menées entre 2008 et 2010 afin de détecter la présence d'occupations paléolithiques et d'évaluer leur état de préservation (850 sondages de 5 à $7 \mathrm{~m}$ de profondeur et atteignant localement $12 \mathrm{~m}$ ).

L'étude des séquences d'Havrincourt s'intègre naturellement dans le cadre des recherches menées depuis plus de vingt ans sur les enregistrements loessiques d'Europe de l'ouest et du nord de la France en étroite relation avec l'approche archéologique des gisements du Paléolithique moyen récent (Antoine, 1991; Antoine et al., 1994, 1998, 2003a,b ; Deschodt et al., 1998; Locht et al., 2002, 2003 ; Vallin \& Masson, 2003 ; Sellier, 2005).

$\mathrm{Du}$ point de vue géomorphologique, le secteur concerné s'étend sur un versant en pente douce exposé

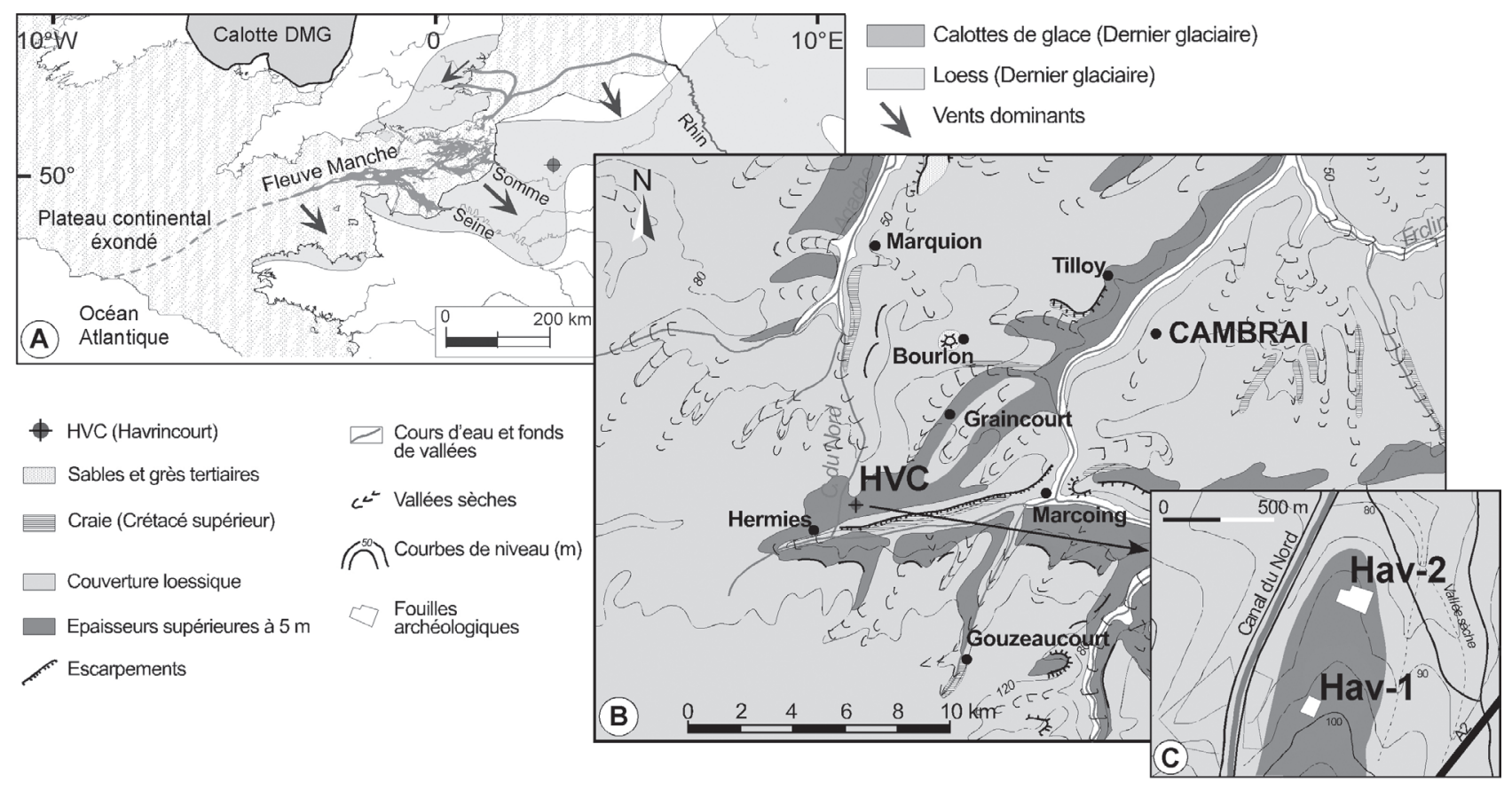

Fig. 1 : Localisation du site d'Havrincourt (A) dans la ceinture loessique ouest européenne (d'après Antoine et al. (2003a) modifié) et (B et C) dans la carte des formations quaternaires du nord de la France (d'après Sommé (1975) modifié).

Fig. 1: Location of the Havrincourt site in (A) the European Loess belt (according to Antoine et al. (2003a) modified) and (B and C) in the map of the Quaternary formations of Northern France (according to Sommé (1975) modified) 
au nord-nord-est, à proximité d'un vallon sec orienté estouest, appartenant au bassin hydrographique de l'Escaut, à une altitude comprise entre 80 et 110 m NGF (fig. 1B et 1C). Au cours du Dernier Glaciaire (Weichselien), cette configuration géomorphologique était favorable à l'accumulation de sédiments d'origine éolienne et plus particulièrement au cours du Pléniglaciaire supérieur (Antoine et al., 1999 ; Antoine, 2002). Dans ce secteur géographique, les séquences loessiques (loess et paléosols), qui reposent sur la craie blanche à Micraster decipiens du Sénonien, peuvent localement dépasser $6 \mathrm{~m}$ d'épaisseur (exceptionnellement plus de $10 \mathrm{~m}$, Marcy et al., 2009). Outre cette couverture de limons quaternaires, des dépôts détritiques tertiaires peu épais sont présents au sommet du versant entre Hermies et Havrincourt, sous forme de poches sableuses résiduelles (Sables du Quesnoy).

A la suite des découvertes réalisées pendant cette phase de prospection, deux campagnes de fouille de grande ampleur (2010 et 2011) ont été menées sur le territoire du village d'Havrincourt (Pas-de-Calais). Elles ont concerné des niveaux paléolithiques préservés dans les dépôts loessiques et les paléosols du Saalien et du Dernier Glaciaire (Weichselien). La fouille du gisement d'Havrincourt s'est déroulée en deux phases (Havrincourt 1 en 2010 et Havrincourt 2 en 2011), sur une durée cumulée de neuf mois. Elle représente une surface globale en fond de fouille de $6000 \mathrm{~m}^{2}$ pour un décaissement total de $6 \mathrm{~m}$ de profondeur en moyenne (Hérisson \& Goval, 2013). Pendant toute la durée de l'opération, un suivi stratigraphique a été assuré en continu (G. Jamet, S. Coutard et P. Antoine). Ce travail a été complété par des campagnes d'échantillonnage systématique destinées aux études sédimentologiques (G. Jamet, P. Antoine) et micromorphologiques (S. Coutard, S. Meszner), mala- cologiques (O. Moine), de magnétisme environnemental (F. Lagroix) et de datations par luminescence optiquement stimulée (OSL) (E. Schmidt et G. Guérin).

Parallèlement à la fouille de quatre niveaux paléolithiques (nommés successivement Hav.1-N3, Hav.2-N0, Hav.2-N1 et Hav.2-N2), cette approche a permis de mettre en évidence une séquence pédosédimentaire riche, complexe et relativement constante sur l'ensemble des secteurs, notamment pour la période du Pléniglaciaire moyen ( 55-35 ka), ainsi qu'une succession unique de plusieurs horizons périglaciaires à grandes fentes particulièrement bien conservés.

L'analyse de la séquence pédosédimentaire d'Havrincourt a débouché sur l'individualisation et la caractérisation d'un grand nombre d'horizons pédologiques (paléosols), de dépôts sédimentaires (loess) et de phénomènes périglaciaires (gleys de toundra et fentes de gel). Compte tenu des données et des connaissances acquises sur les enregistrements régionaux, cette séquence représente un bilan pédosédimentaire essentiellement attribuable au dernier cycle climatique (Eemien-Weichselien) (Antoine et al., 1999). Les données, analyses et datations qui résultent de ces nouvelles recherches nous permettent de proposer Havrincourt comme une nouvelle séquence de référence pour les enregistrements pédosédimentaires du Dernier Glaciaire dans le nord de la France. Parallèlement, elles permettent d'élaborer des schémas de corrélation détaillés avec les enregistrements régionaux (Somme et Nord), ainsi qu'à plus longue distance avec les séquences de Belgique et d'Allemagne. Enfin, sur la base du signal paléoclimatique reconstitué et des datations, des corrélations sont proposées avec les référentiels paléoclimatiques globaux (glaces du Groenland - océan Atlantique).
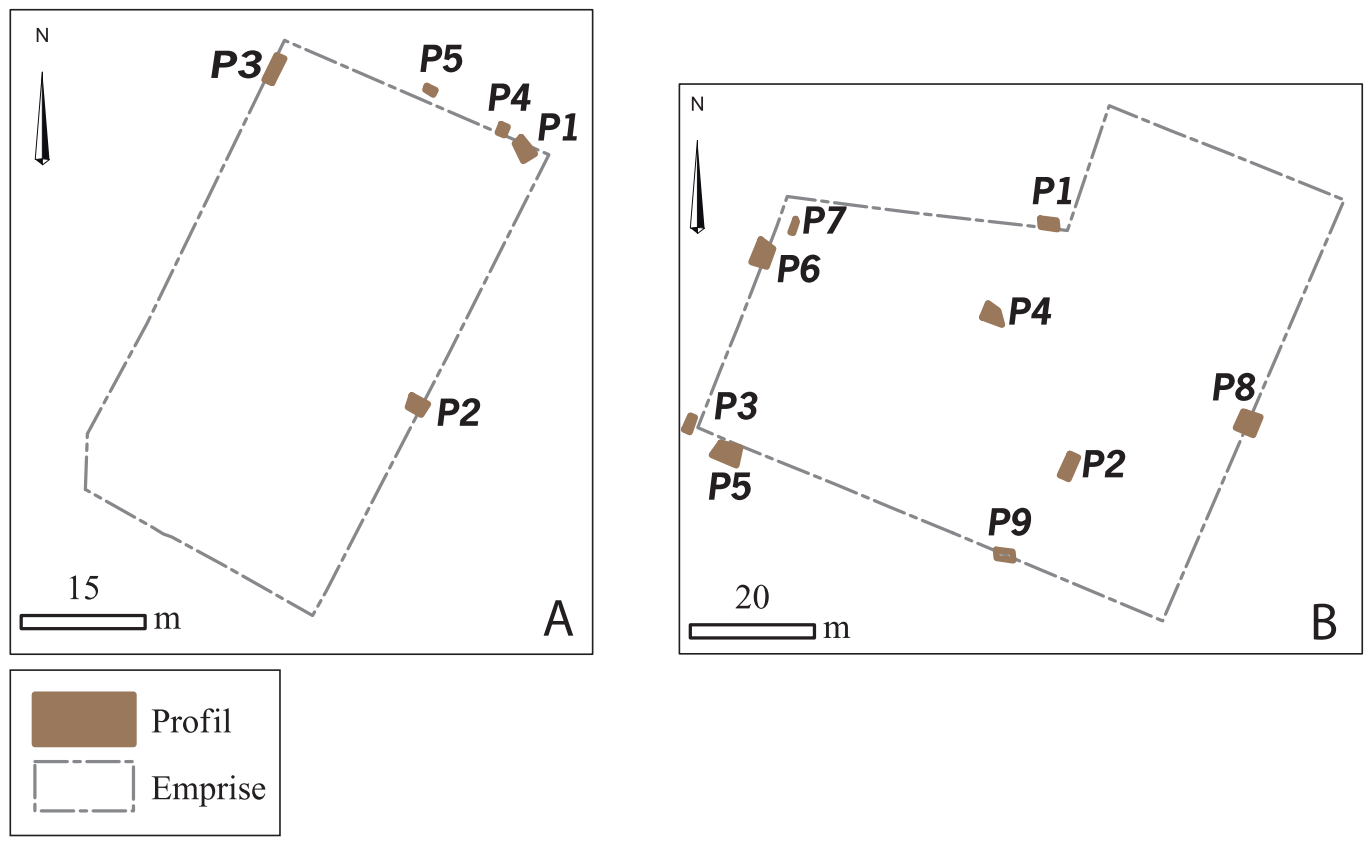

Fig. 2 : Localisation des différents profils stratigraphiques réalisés sur les secteurs Hav.1 (A) et Hav.2 (B).

Fig. 2: Location of the various stratigraphic profiles achieved at Hav.1 (A) and Hav.2 (B). 
Après un exposé détaillé des données stratigraphiques, pédostratigraphiques et des principaux résultats des études biostratigraphiques et des datations, cet article propose une interprétation dynamique, paléoenvironnementale et chrono-climatique de la séquence pédosédimentaire, ainsi qu'une synthèse des données archéologiques.

\section{2 - STRATIGRAPHIE, SÉDIMENTOLOGIE ET DATATION}

\section{1 - MÉTHODOLOGIE DE TERRAIN ET ÉCHANTIL- LONNAGES}

\subsection{1 - Approche globale}

L'approche du contexte chronostratigraphique et paléoenvironnemental des occupations humaines d'Havrincourt se fonde sur la mise en évidence, l'analyse et la datation d'une succession de 17 unités litho-pédostratigraphiques principales individualisées sur le terrain. Cette analyse s'est concentrée sur une série de profils verticaux choisis et dégagés en fonction de la qualité de l'enregistrement et de l'avancement des fouilles (fig. 2). L'ampleur de la surface décapée a permis de réaliser deux grands profils stratigraphiques continus (60 et $70 \mathrm{~m}$ de longueur) qui permettent de suivre les variations latérales des faciès sédimentaires et l'épaisseur de chaque unité (fig. 3). Par ailleurs, le décapage du secteur 2 a permis d'observer, pour la première fois, l'extension horizontale d'un grand réseau de fentes polygonal sur plus de $4000 \mathrm{~m}^{2}$. Les levés détaillés $(10 \mathrm{~cm} / 1 \mathrm{~m})$ et les différentes séries d'échantillons prélevés, en vue des études sédimentologiques et des datations, se sont focalisés sur les profils dénommés Hav.1-P1, P2, P3 et P4 (fig. 2, 4 et 5) et Hav.2-P4, P5, P6 et P9 (fig. 2, 6, 7, 8 et 9). Les échantillons ont été prélevés selon la méthode de la colonne continue avec une résolution de $5 \mathrm{~cm}$ de manière à couvrir l'ensemble de la séquence loessique (fig. 4, 5 et 6).

\subsection{2 - Micromorphologie}

Plusieurs séries de blocs de sédiments et de sols non perturbés (34 au total) ont été prélevées pour la fabrication de lames minces (fig. 4-9). Les lames ont été fabriquées par le laboratoire de pédologie de l'université de Gand (Belgique), l'analyse au microscope polarisant menée par S. Coutard et par le Laboratoire de l'université de Dresde (Allemagne) en ce qui concerne les trois échantillons prélevés dans les loess les plus anciens (S. Meszner).

Un « bloc-colonne » continu (1,4 $\mathrm{m}$ de hauteur) recoupant les unités 5 à 7 a aussi été prélevé dans le secteur 2 (Hav.2-P5) afin de préserver l'intégralité du complexe de sols en vue d'analyses ultérieures.

\subsection{3 - Sédimentologie}

Au total 302 échantillons ( $400 \mathrm{~g}$ par échantillon) ont été prélevés sur les deux secteurs, dont 282 par la méthode de l'échantillonnage en colonne continue avec une résolution de $5 \mathrm{~cm}$ (exceptionnellement 2,5 cm sur Hav.2-P5 au niveau des unités 5 à 7 ), et 20 de manière plus ponctuelle $(\sim 10 \mathrm{~cm})$ dans le profil Hav.2-P9. La méthode de l'échantillonnage en colonne continue permet:

- 1) de conserver la totalité des sédiments et sols dans un but de préservation de l'intégralité des séquences loessiques de référence (« loessothèque » du LGP-Meudon);

- 2) de disposer d'une série continue de données afin de baser l'interprétation des résultats analytiques sur une série au sein de laquelle chaque valeur correspond à une mesure moyennée sur 5 ou 2,5 cm d'épaisseur (limitation du risque de valeur ponctuelle aléatoire).

\subsection{4 - Datations}

Quatre méthodes de datation ont été associées sur les deux secteurs fouillés afin de préciser le contexte chronostratigraphique du site et de caler les différents niveaux d'occupations paléolithiques :

- 1) datation directe des différentes unités loessiques basée sur la méthode OSL (Optically Stimulated Luminescence) sur quartz. A cet effet, 23 échantillons ont été prélevés sans exposition à la lumière à l'aide de tubes en cuivre de $35 \mathrm{x} \sim 140 \mathrm{~mm}$;

- 2) datations ${ }^{14} \mathrm{C}$ (Beta Analytic et Oxford Radiocarbon Accelerator Unit Laboratory) sur os de grand mammifère ( 8 échantillons testés / 2 résultats) et restes osseux de rongeurs provenant des terriers;

-3) datation par thermoluminescence de silex chauffés (artefacts paléolithiques) par la méthode de la thermoluminescence (N. Debenham);

- 4) datations ESR-U/Th sur dents de grands mammifères couplées avec des mesures de dosimétrie des sédiments in situ (J.-J. Bahain, en cours).

\subsection{5 - Anisotropie de la susceptibilité magnétique (ASM)}

Une série de 104 échantillons cubiques $\left(\sim 8 \mathrm{~cm}^{3}\right)$ orientés a été prélevée à intervalles réguliers sur $80 \mathrm{~cm}$ d'épaisseur dans les loess homogènes de l'unité 4 au niveau du profil Hav.2-P5 (fig. 6), afin de mener une étude d'anisotropie magnétique (F. Lagroix). Le prélèvement de chaque cube est effectué à l'aide d'un carottier spécifique à section carrée enfoncé dans la paroi préalablement décapée (deux à trois échantillons pour chaque carottage). Après mesure de l'azimut du carottier et des pendages horizontaux et verticaux à la boussole et à l'inclinomètre, le carottier est retiré de la paroi, puis l'échantillon non perturbé est expulsé par un piston et conservé dans une boîte cubique en polycarbonate. 


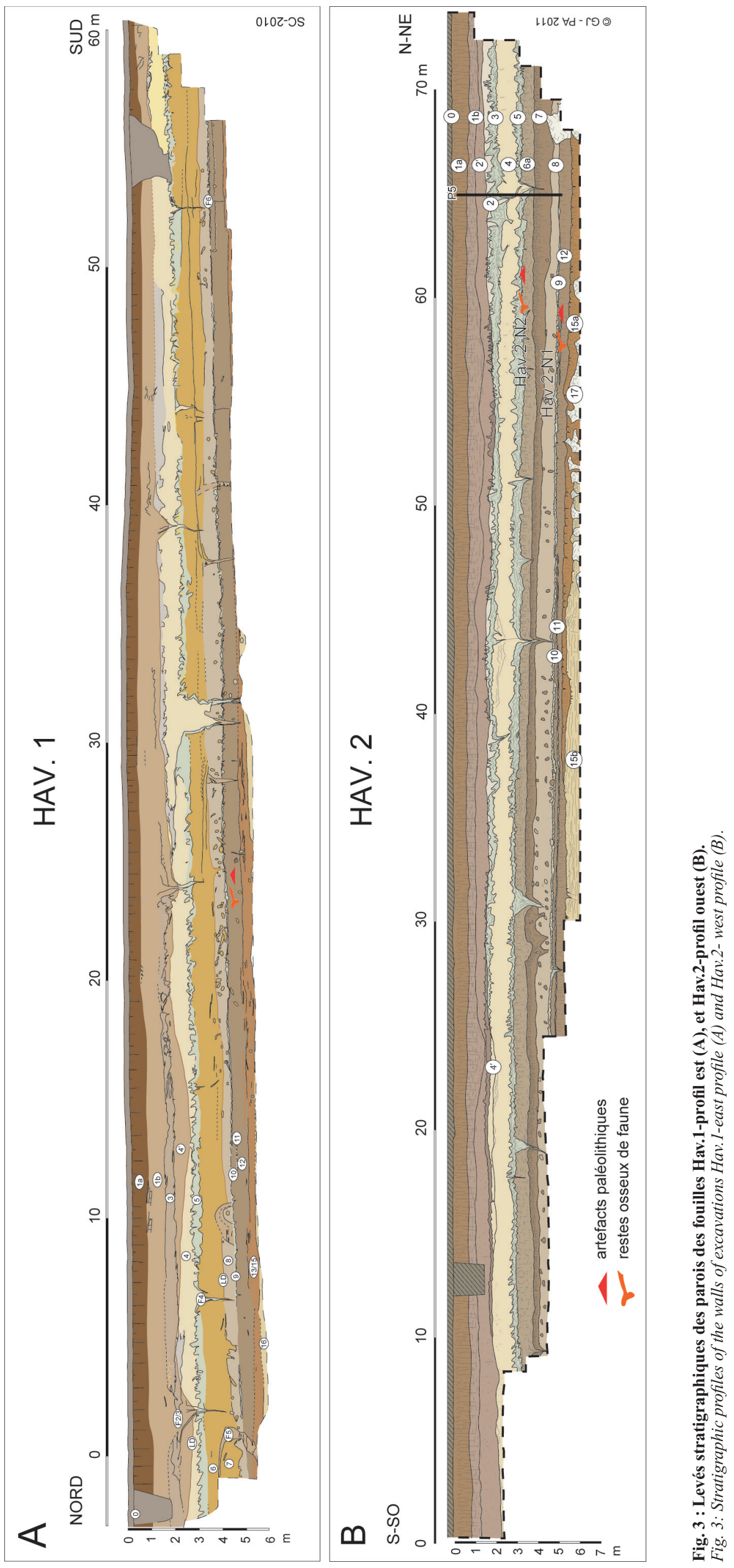




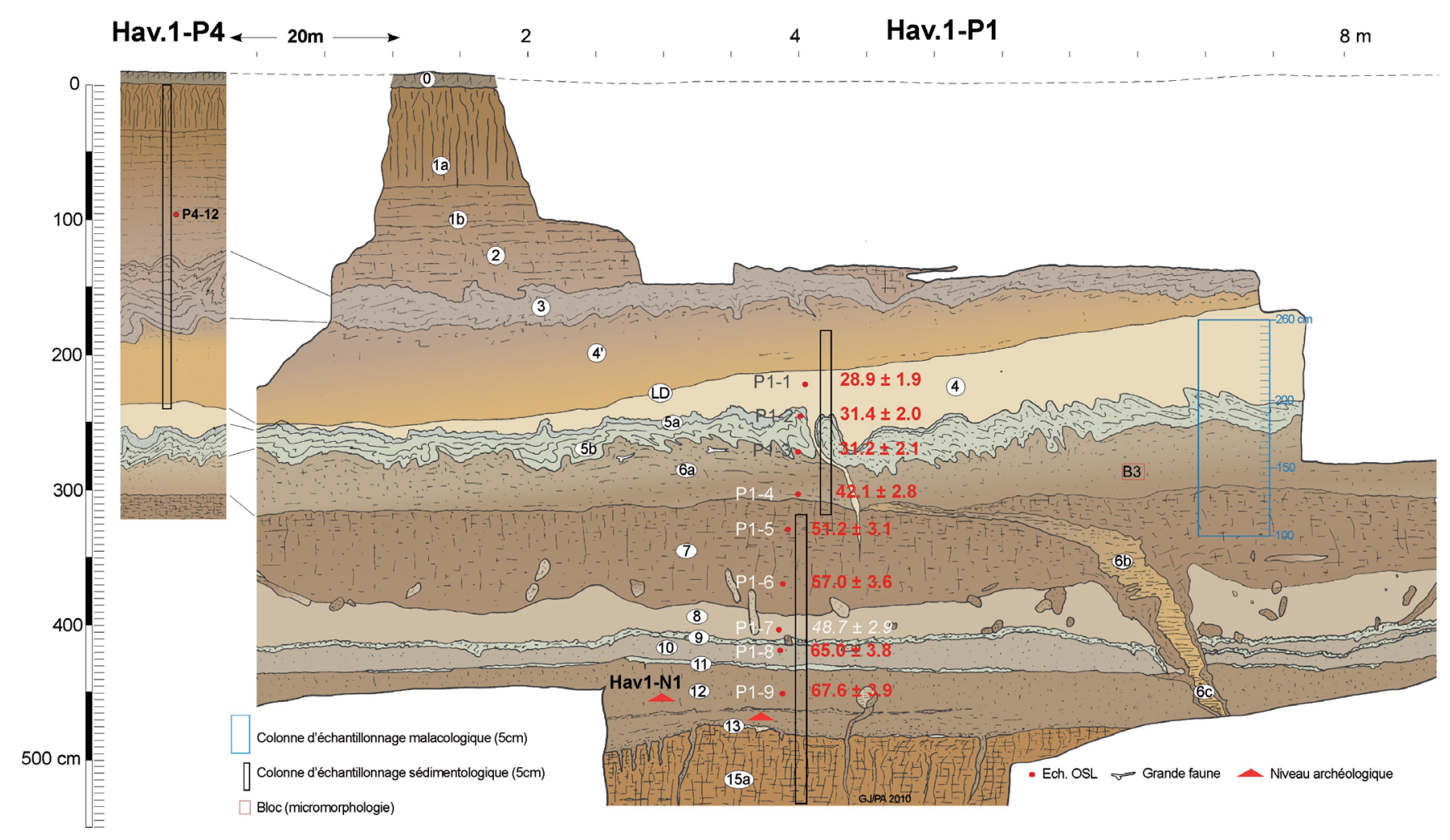

Fig. 4 : Hav.1-P1 et Hav.1-P4. : stratigraphie, échantillonnages et datations OSL.

Fig. 4: Hav.1-P1 and Hav.1-P4: stratigraphy, samplings and OSL datings.

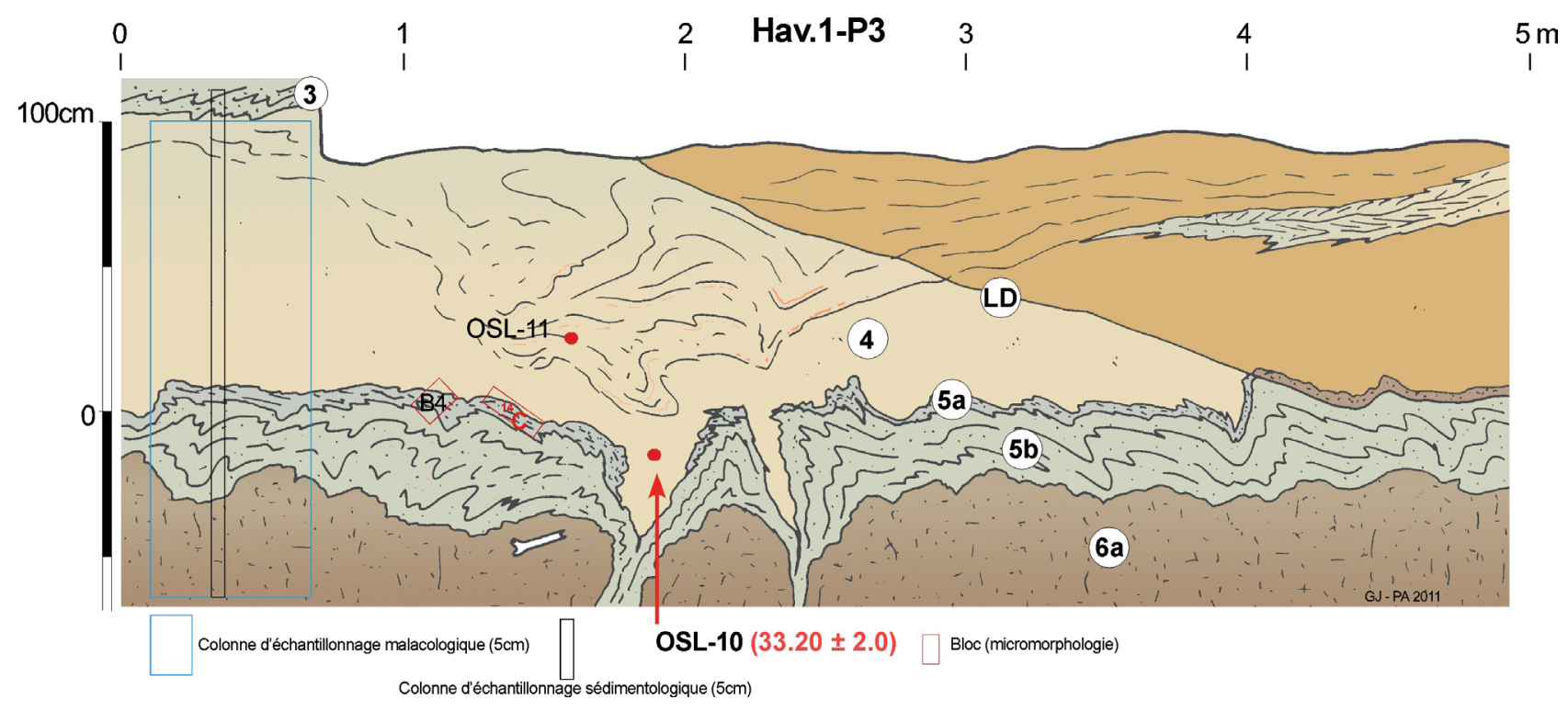

Fig. 5 : Hav.1-P3 : stratigraphie et échantillonnages.

Fig. 5: Hav.1-P3 : stratigraphy and samplings. 


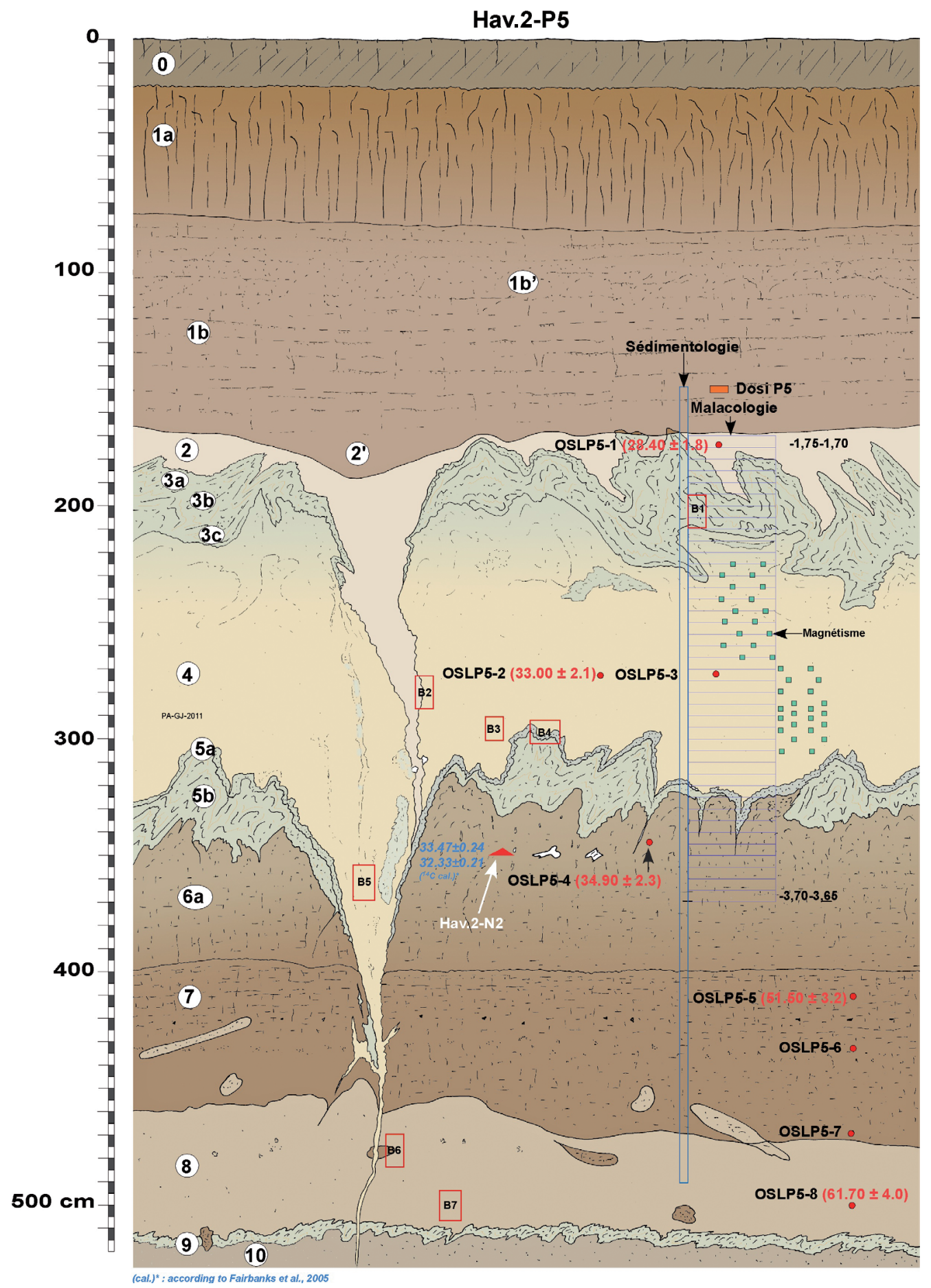

Fig. 6 : Hav.2-P5 : stratigraphie, échantillonnages et datations OSL.

Fig. 6: Hav.2-P5: stratigraphy, samplings and OSL datings.

\subsection{6 - Malacologie}

Pour cette approche, deux colonnes de 33 et 40 échantillons ont été respectivement prélevées sur les profils Hav.1-P1 (fig. 3 et 4) et Hav.2-P5 (fig. 6). Ces échantillons de 10 litres de sédiment chacun ont été prélevés en colonne continue tous les $5 \mathrm{~cm}$ (colonnes d'environ
$60 \mathrm{~cm}$ de large découpées en tranches successives de $5 \mathrm{~cm}$ ), en parallèle avec les échantillons et mesures destinés aux analyses sédimentologiques. La colonne d'échantillonnage du profil Hav.1-P1 débute dans un horizon limoneux (unité 7) du complexe de sols bruns, recoupe un autre horizon de ce complexe (unités 6a), puis un gley de toundra calcaire (unité 5), et se termine 


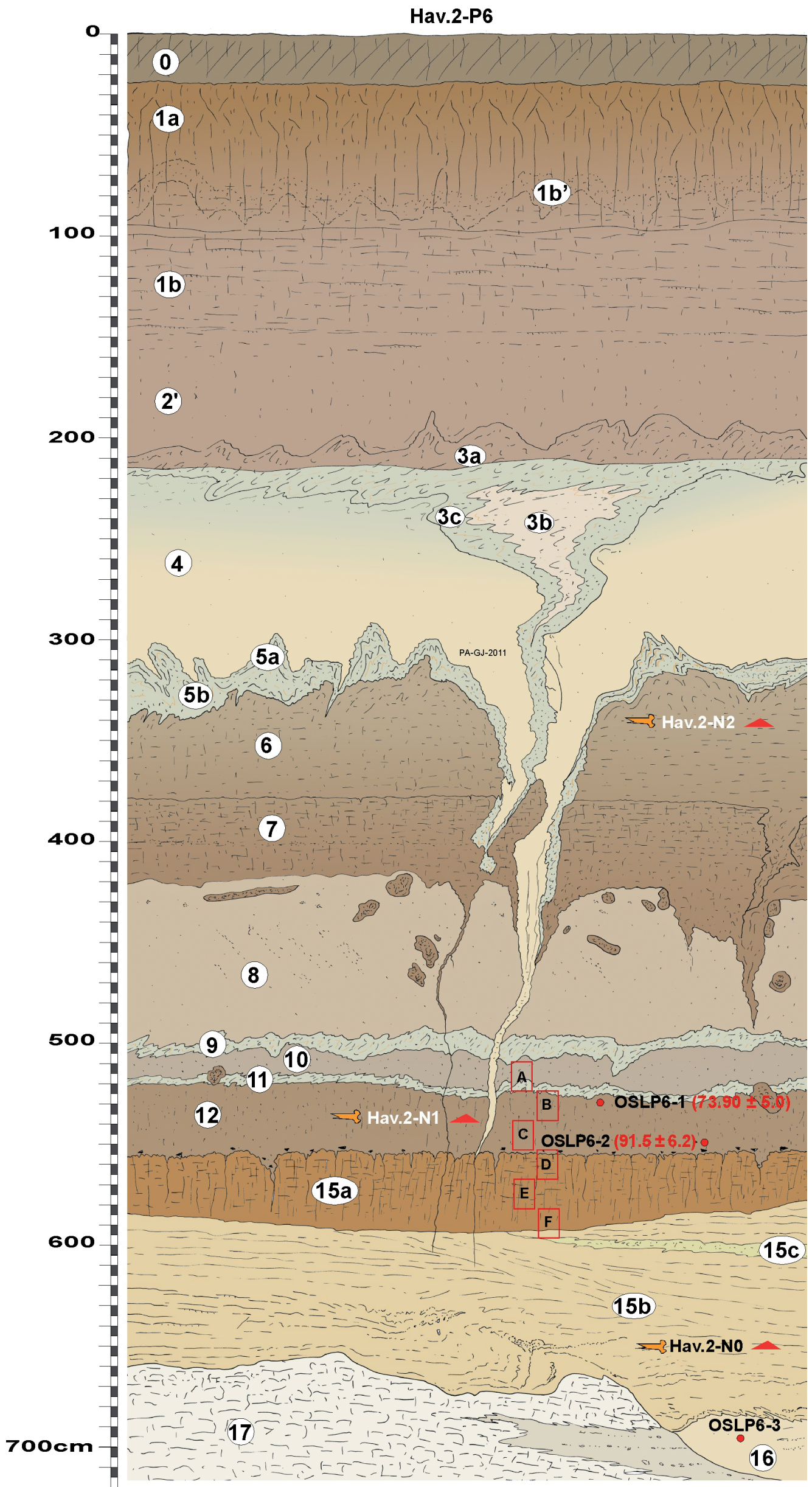

Fig. 7 : Hav.2-P6 : stratigraphie, échantillonnages et datations OSL.

Fig. 7: Hav.2-P6: stratigraphy, samplings and OSL datings. 


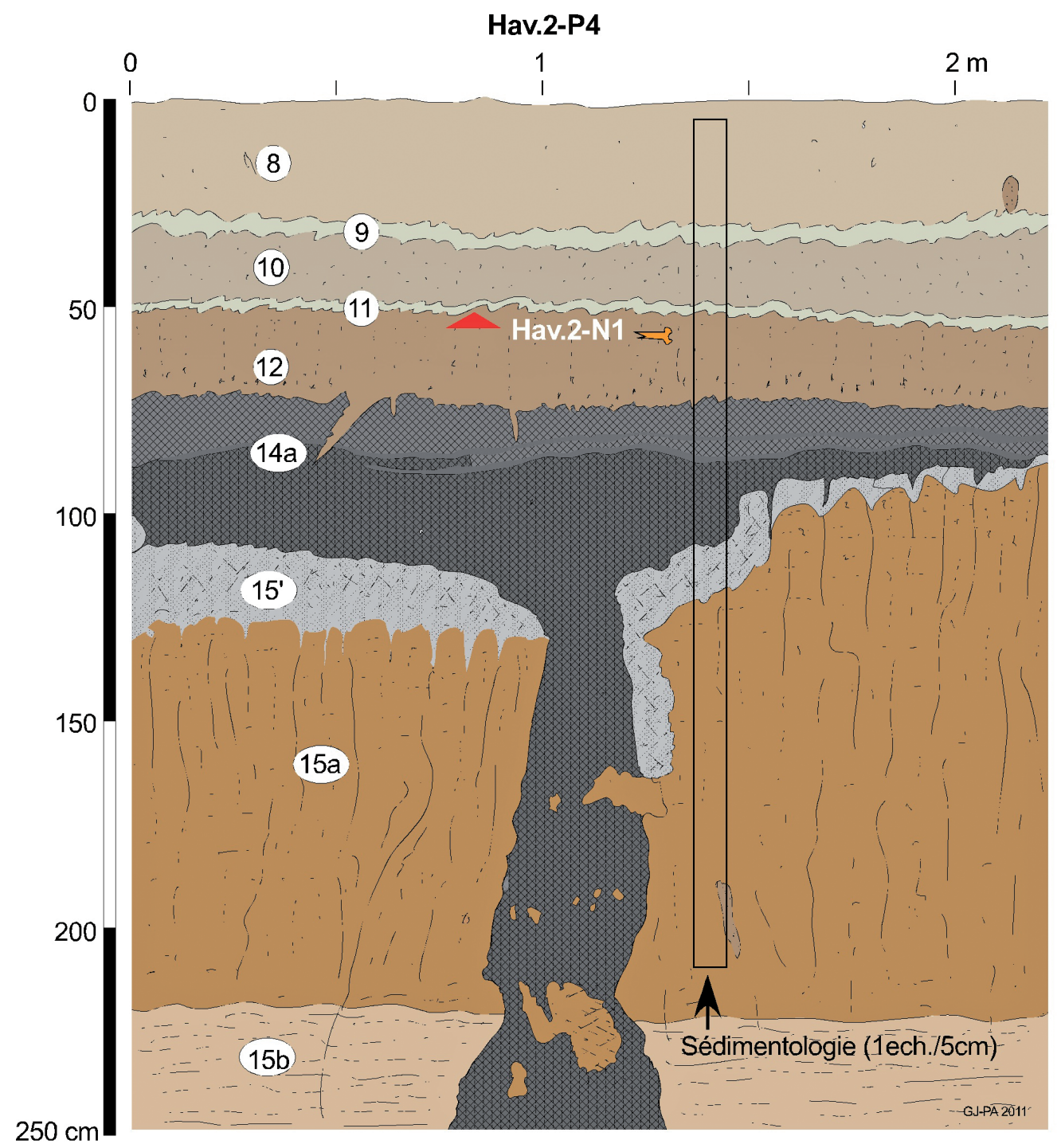

Fig. 8 : Hav.2-P4 : stratigraphie et échantillonnages.

Fig. 8: Hav.2-P4 : stratigraphy and samplings.

au sommet d'un loess carbonaté homogène (unité 4) sous la limite de décarbonatation supérieure (LD). La colonne du profil Hav.2-P5 débute un peu plus haut dans l'unité supérieure du complexe de sols bruns légèrement carbonatée (unité $6 a$ ), recoupe les unités 5 et 4 , puis un gley de toundra calcaire complexe dédoublé sus-jacent (unités 3a-3c), pour se terminer au sommet de cette unité dans sa zone de transition-mélange avec la base du loess carbonaté homogène sommital (unité 2) juste sous la limite de décarbonatation supérieure (LD-1).

\section{2 - STRATIGRAPHIE ET DESCRIPTION DES UNITÉS}

Le relevé systématique et la corrélation des profils réalisés sur les deux secteurs de fouille, couplés à l'analyse sédimentologique, ont abouti à la mise en évidence d'une succession de 17 unités principales, résumée dans le tableau 1 et la figure 10 .

Du point de vue litho- et morpho-stratigraphique, l'analyse des unités stratigraphiques des différents profils d'Havrincourt a permis de mettre en évidence une épaisse formation quaternaire, dont l'épaisseur cumulée totale atteint environ $7,5 \mathrm{~m}$. La synthèse des caractéristiques pédosédimentaires et de la géométrie des différentes unités et horizons repères périglaciaires débouche sur la production d'une séquence synthétique résultant de la corrélation des différents profils étudiés (fig. 10). L'interprétation chrono-climatique des différents faciès est basée sur la synthèse de l'analyse des macro- et des microfaciès, et des données sédimentologiques analytiques et bioclimatiques (mollusques, mammifères). Cette démarche permet de dégager un certain nombre de grands ensembles pédosédimentaires correspondant à des cycles dépôt-pédogenèse d'ampleurs variables.

D'une manière générale, la mise en évidence d'un horizon de sol de rang interglaciaire typique partiellement tronqué dans tous les profils d'Havrincourt vers cinq mètres de profondeur (unité 15), permet de scinder l'enregistrement en deux grandes séquences de type glaciaire-interglaciaire très inégalement représentées. 


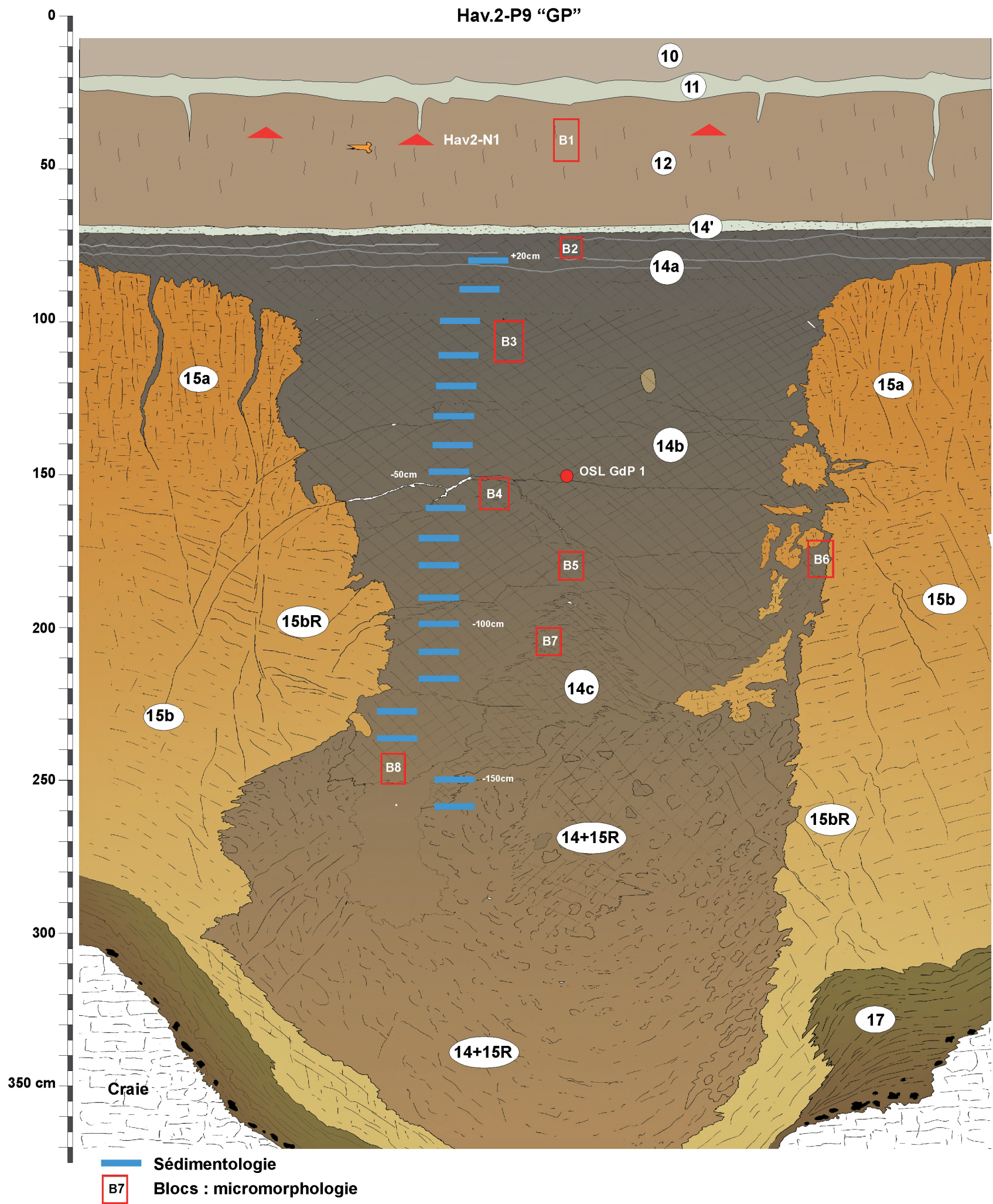

Fig. 9 : Hav.2-P9 « grande poche » : stratigraphie et échantillonnages.

Fig. 9: Hav.2-P9 "large dissolution sinkhole": stratigraphy and samplings.

La séquence 1 est composée des unités 17 à $15 \mathrm{a}(\sim 2,5 \mathrm{~m}$ cumulés), et la séquence 2 des unités 14 à 1 ( $5 \mathrm{~m}$ cumulés ; l'unité 0 étant l'horizon de labour actuel).

L'interprétation chronostratigraphique de la séquence d'Havrincourt développée dans la partie 5 se fonde sur la synthèse des données stratigraphiques, sédimentologiques, bioclimatiques et géochronologiques obtenues dans le cadre d'une équipe interdisciplinaire coutumière de cette approche des milieux loessiques. Elle s'intègre par ailleurs dans le système pédosédimentaire et chronostratigraphique du dernier cycle climatique progressivement développé et complété pour la France septentrionale sur la base de plus de vingt ans d'observations (Antoine, 1988 ; Antoine et al., 2001, 2002a, 2009, 2013). 


\section{HAVRINCOURT 2010-2011}

Structures périglaciaires
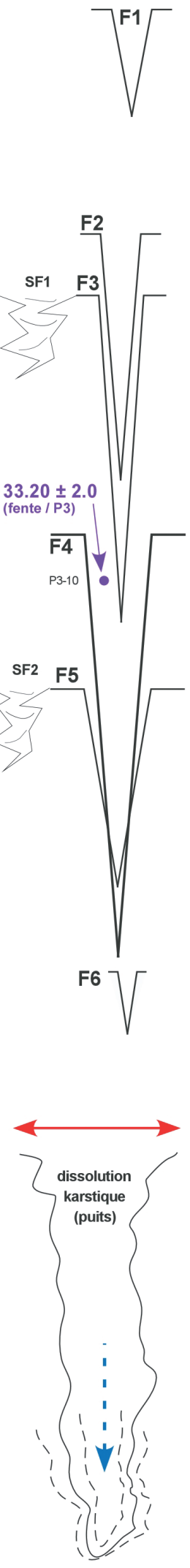

Profil synthétique
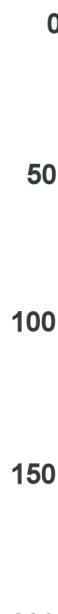

35

($$
400
$$$$
5
$$

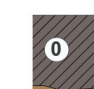

1 a$$
200
$$$$
250
$$$$
300
$$$$
350
$$
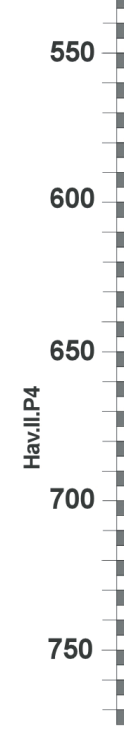

Datations

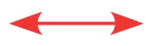

OSL (quartz)

Hav.1-P1 (noir)

Hav.1-P3 (mauve)

- Hav 2-P5 (rouge)

Hav 2-P6 (gris)

폴

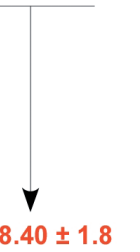

$28.40 \pm 1.8$

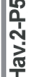

$\longleftarrow 33.00 \pm 2.1$

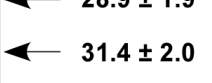

$-31.2 \pm 2.1$

$\longleftarrow 34.90 \pm 2.3^{33.47 \pm 0.24}$

$32.33 \pm 0.21$

(cal.)

Complexe de sols arctiques

$\longleftarrow \quad 42.10 \pm 2.8$

士 $51.50 \pm 3.20 \pm 3.2$

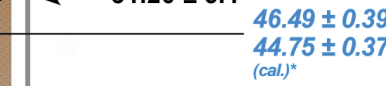

Complexe de sols brun boréaux

$\longleftarrow 57.00 \pm 3.6$

$\longleftarrow 61.70 \pm 4.0$

Hav.1.P1

$\longleftarrow 65.00 \pm 3.8$

$\longleftarrow 73.90 \pm 5.0$

— $67.60 \pm 3.9$

$\longleftarrow 91.50 \pm 6.2$

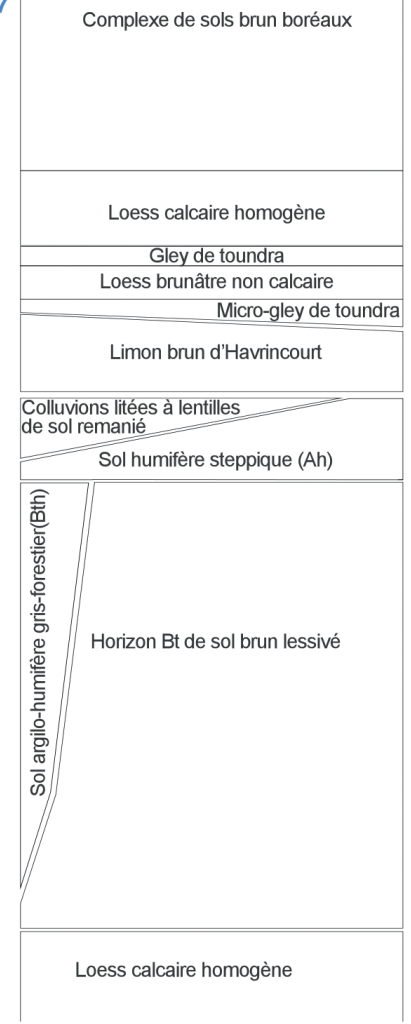

Fig. 10 : Havrincourt 1 et 2 : Synthèse pédo-stratigraphique, datations et structures périglaciaires.

Fig. 10: Havrincourt 1 and 2: pedostratigraphic synthesis, datings and periglacial features. 


\begin{tabular}{|c|c|c|}
\hline Unités & Description & Eléments d'interprétation \\
\hline 0 & Limon brun gris à traces de racines, limite basale franche. & Horizon Ap du sol de surface (Labour). \\
\hline 1 & $\begin{array}{l}\text { (1a) Limon brun foncé argileux à brun orangé compact (10 YR 4/3 5/3), non calcaire, à } \\
\text { structure prismatique grossière. Bioturbation importante soulignée par des pédotubules à } \\
\text { enduits silto-argileux brun noir : agricutanes. } \\
\text { (1b) Limon loessique brun clair à beige }(7,5 \text { YR } 6 / 45 / 4) \text {, non calcaire, à structure en doublets } \\
\text { (litages millimétriques soulignés par des alternances de lits millimétriques bruns et beige } \\
\text { grisâtre ondulés et plus fins vers la base). Nombreux pédotubules à colmatage argilo- } \\
\text { humique grisâtre (agricutanes). } \\
\text { (1b') (Fig. } 5:-100 \mathrm{~cm}) \text { : petit horizon argileux brun grisâtre, non calcaire, irrégulier. }\end{array}$ & $\begin{array}{l}\text { (1a) Horizon Bt du sol brun lessivé de surface } \\
\text { (Luvisol). (1b) loess décarbonaté à faciès de limon } \\
\text { à doublets. } \\
\mathbf{1 b} \text { intègre localement un mince horizon déformé } \\
\text { (langues) correspondant à un gley de toundra } \\
\text { altéré (décarbonaté) (1 } \mathbf{1} \text { ') associé à de rares } \\
\text { pseudomorphoses de grandes fentes à coin de } \\
\text { glace (niveau } \mathbf{F}-\mathbf{1} \text { ). }\end{array}$ \\
\hline 2 & $\begin{array}{l}\text { (2) Loess calcaire brun clair, homogène, le plus souvent décarbonaté (faciès 2'). Quelques } \\
\text { traces de racines et agricutanes. }\end{array}$ & $\begin{array}{l}\text { (2) Loess calcaire typique homogène. (2') faciès } \\
\text { décarbonaté par la pédogenèse du sol de surface } \\
\text { (LD-1). }\end{array}$ \\
\hline 3 & $\begin{array}{l}\text { (3a / 3c) Horizons hydromorphes gris à linéoles oxydées orange éparses. Dans plusieurs } \\
\text { profils, la pédogenèse du sol } 1 \text { affecte le sommet de (3a). } \\
\text { Deux niveaux de grandes fentes à coin de glace de type ice-wedges, à maille décamétrique, ont } \\
\text { été observés en liaison avec les gleys 3a et 3c (F-2 et F-3). Des structures de fusion localisées } \\
\text { (SF-1), caractérisées par des remplissages de loess laminé à structures de ruissellement } \\
\text { intense (stratifications obliques) ont été observées en liaison avec le niveau F-2. } \\
\text { (3b) Loess calcaire jaunâtre homogène et peu épais essentiellement préservé dans le } \\
\text { colmatage des fentes du niveau F-3. }\end{array}$ & $\begin{array}{l}\text { Horizon hydromorphe périglaciaire complexe } \\
\text { résultant de la superposition de deux niveaux } \\
\text { de gleys de toundra cryoturbés séparés par un } \\
\text { mince dépôt loessique (3b) : (gleysol) à langues de } \\
\text { gélifluxion (permafrost / ice-wedges F3). }\end{array}$ \\
\hline 4 & $\begin{array}{l}\text { (4) Loess calcaire jaune à beige (10 YR 6/6) pulvérulent et homogène, à fines taches } \\
\text { d'oxydation sommitales (linéoles) et structure lamellaire fine. Horizon affecté localement } \\
\text { par la décarbonatation liée au sol de surface (LD1) (faciès } 4^{\prime} \text { ). }\end{array}$ & $\begin{array}{l}\text { (4) Loess calcaire typique homogène à structure } \\
\text { de gel-dégel }\end{array}$ \\
\hline 5 & $\begin{array}{l}\text { (5a) Horizon loessique grisâtre à lamines blanchâtres irrégulières, débris noirâtres épars et } \\
\text { nombreuses concrétions calcaires }(<1 \mathrm{~cm}) \text {. Un réseau de grandes fentes de type ice-wedges, } \\
\text { à maille décamétrique est associé à cet horizon (niveau principal F-4). } \\
\text { (5b) Loess calcaire hydromorphe gris verdâtre cryoturbé à taches d'oxydation. Les unités } \\
\text { 5a-5b apparaissent fluées sur les parois des pseudomorphoses de fentes (faciès : limon gris } \\
\text { à brun, déformé, à linéoles oxydées oranges). }\end{array}$ & $\begin{array}{l}\text { (5) Horizon périglaciaire de type gley de toundra } \\
\text { (gleysol) à langues de gélifluxion associé au } \\
\text { développement d'un pergélisol (ice-wedges, } \\
\text { niveau principal F-4). }\end{array}$ \\
\hline 6 & $\begin{array}{l}\text { (6a) Limon argileux brun gris à taches et enduits orangés, compact, à forte structure } \\
\text { granulaire à lamellaire }(4-6 \mathrm{~mm}) \text {, Nombreuses concrétions ferromanganiques }(\varnothing \pm 1 \mathrm{~mm}) \\
\text { à la base. } \\
\text { (6b) Loess brun calcaire homogène. } \\
\text { (6c) Limons ruisselés calcaires jaunes à brun-jaune à stratifications entrecroisées. } \\
\text { Un niveau de grandes fentes en coin déformées s'ouvre au sommet de 6a (niveau F-5). }\end{array}$ & $\begin{array}{l}\text { (6a) Horizon de type sol brun-arctique (gelic } \\
\text { cambisol), à sol de prairie arctique développé sur } \\
\text { loess carbonaté } \\
\text { (6b) Loess correspondant au colmatage terminal } \\
\text { des pseudomorphoses de grandes fentes à coins } \\
\text { de glace du réseau F-5. } \\
\text { (6c) Faciès hétérogène correspondant au } \\
\text { colmatage par ruissellement de la base des } \\
\text { grandes fentes à coins de glace (fluage) du niveau } \\
\text { F-5. Niveau archéologique (Hav.2-N2) }\end{array}$ \\
\hline 7 & $\begin{array}{l}\text { Limon argileux brun à brun orangé }(7,5 \text { YR } 5 / 65 / 8) \text {, compact et homogène, non calcaire, } \\
\text { à structure polyédrique à lamellaire }(3-8 \mathrm{~mm}) \text {. Les surfaces des agrégats comportent } \\
\text { localement des enduits ferromanganiques noirâtres et d'oxydes de fer orangés. La structure } \\
\text { lamellaire disparaît progressivement en profondeur. Cet horizon montre de nombreuses } \\
\text { traces de terriers de grande taille }(\varnothing 10-15 \mathrm{~cm}) \text { à colmatage varié (loess } 8 / \text { sol } 6) \text {. Certains } \\
\text { terriers ont livré des restes de rongeurs très bien conservés. }\end{array}$ & $\begin{array}{l}\text { Horizon Bw de sol brun boréal (cambisol) du type } \\
\text { Sol de Saint-Acheul-Villiers-Adam. } \\
\text { Cette unité est affectée par les processus de gel- } \\
\text { dégel intense (structures résiduelles de la glace } \\
\text { de ségrégation) et un permafrost continu (ice- } \\
\text { wedges). Niveau F-5. }\end{array}$ \\
\hline 8 & $\begin{array}{l}\text { Loess brun-jaune (7,5 YR 6/4 5/4), compact et homogène, calcaire. Manchons racinaires } \\
\text { calcifiés et granules de craie épars à la base. Cet horizon à taches de manganèse noires se } \\
\text { distingue par l'abondance de terriers de grande taille (krotovina } / \varnothing 10-30 \mathrm{~cm} \text { ) à colmatés } \\
\text { par du matériel brun déstructuré (structure granulaire) provenant du sol de l'unité } 7 \text {. }\end{array}$ & $\begin{array}{l}\text { Loess calcaire incluant des granules de craie } \\
\text { remaniés à partir du substrat local et dont la } \\
\text { partie supérieure est affectée par la pédogenèse } \\
\text { du sol } 7 \text { (décarbonatation). }\end{array}$ \\
\hline 9 & $\begin{array}{l}\text { Horizon hydromorphe, non calcaire, grisâtre déferrifié à géométrie " boudinée » non } \\
\text { calcaire. Concrétions calcaires (poupées de } 1 \mathrm{~cm} \text { ) à la base et des pédotubules. Un niveau } \\
\text { de fines fentes de gel à colmatage loessique s'ouvre au sommet de cette unité (F-6). }\end{array}$ & $\begin{array}{l}\text { Petit horizon périglaciaire de type gley de toundra } \\
\text { cryoturbé (gelic gleysol) associé à un niveau de } \\
\text { fines fentes à colmatage loessique (F-6). }\end{array}$ \\
\hline 10 & $\begin{array}{l}\text { Limon loessique brun homogène, faiblement carbonaté, à nombreux nodules ferro- } \\
\text { manganiques et traces de biotubules. Cet horizon est affecté par les déformations de l'unité } \\
\text { sus-jacente. }\end{array}$ & $\begin{array}{l}\text { Loess non calcaire (décarbonaté) faiblement } \\
\text { déformé par des processus de gel-dégel }\end{array}$ \\
\hline 11 & $\begin{array}{l}\text { Petit horizon loessique hydromorphe gris brun diffus et irrégulier à imprégnations ferriques } \\
\text { rougeâtres. }\end{array}$ & $\begin{array}{l}\text { " micro-gley " de toundra avec engorgement } \\
\text { temporaire de la surface (gelic gleysol). }\end{array}$ \\
\hline 12 & $\begin{array}{l}\text { Limon argileux brun à brun grisâtre, homogène, non calcaire à structure compacte dans la } \\
\text { partie sommitale. Concrétions ferromanganiques }(\emptyset \pm 1 \mathrm{~mm}) \text { manchons racinaires calcifiés et } \\
\text { pédotubules ovalaires épars. }\end{array}$ & $\begin{array}{l}\text { Horizon limoneux d'origine partiellement } \\
\text { colluviale affecté par une pédogenèse peu } \\
\text { développée (gelic cambisol ou sol de prairie } \\
\text { arctique). Niveau archéologique (Hav.1-N3 et } \\
\text { Hav. 2-N1) }\end{array}$ \\
\hline
\end{tabular}

Tab. 1 : Description des unités stratigraphiques.

Tab. 1: Description of the stratigraphic units. 


\begin{tabular}{|c|c|c|}
\hline 13 & $\begin{array}{l}\text { Limons non calcaires fortement lités, jaune à brun, irrégulier à lentilles de (14) à (15), rares } \\
\text { fentes de gel irrégulières. }\end{array}$ & $\begin{array}{l}\text { Limons lités résultant de l'érosion et du } \\
\text { remaniement par gélifluxion et ruissellent sur } \\
\text { versant de la partie inférieure de la séquence } \\
\text { (unités } 14 \text { à 16). }\end{array}$ \\
\hline $14^{\prime}$ & orizon silteux irrégulier à nombreuses concrétions ferromanganiques (Fig. 8). & $\begin{array}{l}\text { Horizon « blanchi » marquant localement la limite } \\
\text { supérieure de l'ensemble } 14 .\end{array}$ \\
\hline 14 & $\begin{array}{l}\text { (14a) Limon gris foncé humifère à structure granulaire à polyédrique La partie sommitale } \\
\text { est marquée par un léger cailloutis (silex), des concrétions ferromanganiques et des } \\
\text { bioturbations sur } 5 \text { à } 10 \mathrm{~cm} \text {. } \\
\text { (14b) Limon argileux brun à brun noir compact à forte structure polyédrique à prismatique, } \\
\text { enduits ferromanganiques sur fissures et illuviations d'argile brun-noir sur pores, enduits } \\
\text { silteux blanc-gris lités dans les biopores, nombreuses cavités (logettes : } 1 \mathrm{~cm} \text { ) à remplissage } \\
\text { de pellets argileux et (ou) de silts blancs (vers). }\end{array}$ & $\begin{array}{l}\text { (14a) Horizon gris humifère de type sol steppique, } \\
\text { dégradé dans la partie sommitale. } \\
\text { (14b) Faciès de sol gris forestier typique } \\
\text { particulièrement bien conservé dans le } \\
\text { remplissage de structures de dissolution localisées } \\
\text { (puits) affectant le substratum crayeux (Fig. } 7 \text { et } 8 \text { ). } \\
\text { L'ensemble 14a-b-c est le plus souvent démantelé } \\
\text { par une érosion intense. }\end{array}$ \\
\hline $15^{\prime}$ & $\begin{array}{l}r \text { à nombreuses concrétions ferromanganiques remplissant les } \\
\text { a. }\end{array}$ & $\begin{array}{l}\text { Horizon " blanchi " à glosses développé au } \\
\text { sommet du sol 15a }\end{array}$ \\
\hline 15 & $\begin{array}{l}\text { (15a) Limon argileux non calcaire, compact, brun orangé à rougeâtre à forte structure } \\
\text { prismatique grossière. La partie sommitale est marquée par des glosses à remplissage } \\
\text { grisâtre plus ou moins humifère }\left(15^{\prime}\right) \text {. } \\
\text { (15b) Limon brun clair, compact, décarbonaté, à structure en doublets irrégulière (ondulée) } \\
\text { à faible pendage, localement structurations obliques. Chaque doublet }( \pm 1 \mathrm{~cm}) \text { se caractérise } \\
\text { par la succession d'un lit brun clair et d'un lit brun foncé coiffé d'un liseré de manganèse } \\
\text { noir. } \\
\text { (15c) Faciès hydromorphe de (15b) matérialisé par des passées gleyifiées à langues de } \\
\text { gélifluxion. } \\
\text { (15d) Limon loessique compact, non calcaire, brun clair homogène. }\end{array}$ & $\begin{array}{l}\text { (15a) Horizon Bt profond (tronqué) et fortement } \\
\text { dégradé du sol brun lessivé de type Horizon Bt } \\
\text { interglaciaire } \\
\text { (15b) Limon loessique, décarbonaté à doublets, } \\
\text { localement nettement stratifié (remaniement } \\
\text { antérieur à la pédogenèse interglaciaire). Partie } \\
\text { inférieure homogène (15d) correspondant au } \\
\text { faciès décarbonaté du loess } 16 . \\
\text { Cette unité montre localement un faciès } \\
\text { hydromorphe (15c) en bandes irrégulières } \\
\text { correspondant à un gley de toundra mal conservé. }\end{array}$ \\
\hline 16 & Limon & $\begin{array}{l}\text { Loess calcaire typique homogène. } \\
\text { Niveau archéologique Hav.2-N0. }\end{array}$ \\
\hline 17 & $\begin{array}{l}\text { Craie fracturée en petits blocs, localement matrice crayeuse plus fine. Contact supérieur } \\
\text { localement marqué par des diapirs et des injections traduisant un épisode de cryoturbation } \\
\text { intense (structure en diapirs / cryo-injection) au contact de } 16 .\end{array}$ & $\begin{array}{l}\text { Substratum crayeux affecté par les processus de } \\
\text { remaniement par gélifluxion sur versant (Head } \\
\text { crayeux) et de cryoturbation intenses. }\end{array}$ \\
\hline
\end{tabular}

Tab. 1 (suite)

Tab. 1 (continuation)

\subsection{1 - Micromorphologie (S.C. / S.M.)}

\subsubsection{1 - Loess supérieurs et gleys de toundra (unités 5} à 3)

Les gleys et loess calcaires de la partie supérieure de la séquence stratigraphique ont été observés à partir d'une série de prélèvements issus du profil Hav.2-P5 (fig. 6), et par un échantillon prélevé sur le profil Hav.1-P3 (fig. 5).

Le gley supérieur (unité 3a / lame Hav.2-P5-1) est développé sur un limon loessique calcaire bien classé contenant quelques granules de craie. Une microstructure de gel-dégel, de type lamellaire à la base de l'échantillon, très régulière y est très bien exprimée. Des ovoïdes de gélifluxion (agrégats ovoïdes entourés par une pellicule de particules fines) sont intégrés dans le sédiment à l'intérieur des lamelles. Ils pourraient témoigner d'une phase de gel-dégel antérieure. Certains agrégats plus gros et orangés, décarbonatés, sont également présents : ils proviennent de l'horizon sous-jacent (unité 6 sup.). Dans les deux tiers supérieurs de la lame, on passe à une microstructure lenticulaire à granulaire, fine, intégrant de petits agrégats micritiques arrondis (fig. 11-I). Quelques sphérolites (granules calcitiques) de lombric et quelques fragments de mollusques sont dispersés dans le sédiment.
La lame Hav.2-P5-B3 (bloc 3 du profil 5) correspond au loess calcaire de l'unité 4 . Il s'agit d'un loess typique avec très peu de fraction fine; les carbonates sont détritiques (« boulettes » micritiques de la taille des limons) et les argiles sont rares. L'échantillon Hav.2-P5-B5 provenant du colmatage d'une grande fente de gel montre un limon loessique plus perturbé contenant de nombreuses micro-concrétions ferro-manganiques. Les sphérolites de lombrics y sont nombreux.

L'unité 5 (gley de toundra inférieur) est représentée par les lames Hav.2-P5-B4 et Hav.1-P3-B1. La lame Hav.1P3-B1 (unité 5) montre un limon loessique carbonaté, dont la masse basale grisâtre contient des microparticules noirâtres de type matière organique végétale. L'activité biologique est intense. L'hydromorphie est nettement exprimée, avec une ferruginisation de la masse basale par bandes, des revêtements et des hypo-revêtements ferrugineux dans les chenaux. Des coquilles de mollusques sont visibles. Dans la lame Hav.2-P5-B4, il s'agit également d'un limon loessique carbonaté, avec une masse basale carbonatée et poussiéreuse irrégulièrement répartie. Des particules de matière organique sont visibles dans la partie sommitale. Contrairement au gley (unité 3), aucune structure de gel-dégel n'est visible dans l'unité 5 (destruction par gélifluxion?). 


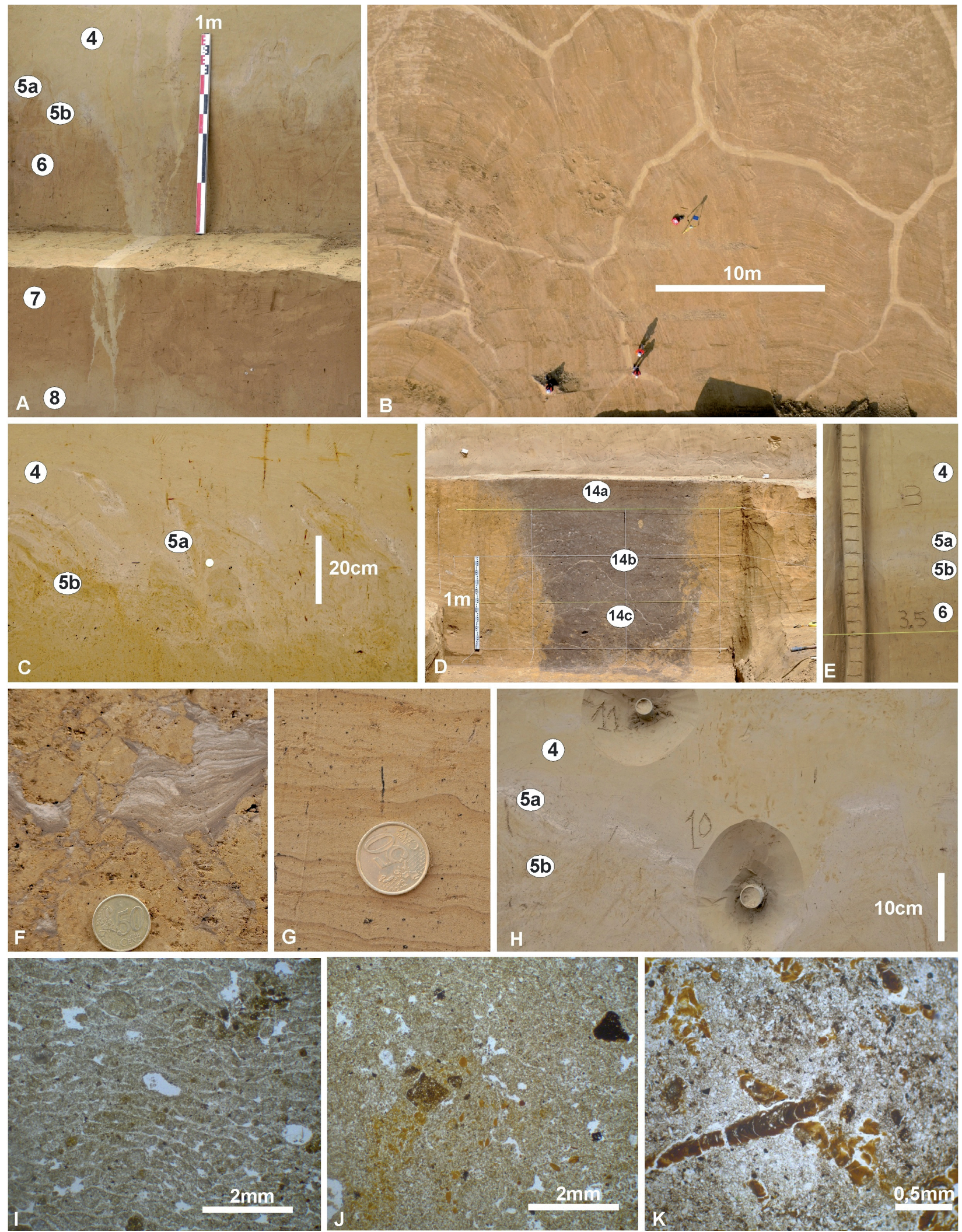

Fig. 11 : Hav.2 - Stratigraphie, sédimentologie et structures périglaciaires (voir tableau 1 pour la description des unités).

A/ Hav.2-P5 : grande fente en coin à remplissage loessique (niveau principal F-4) recoupant le « Complexe de sols d'Havrincourt » (cliché P. Antoine). $\mathrm{B}$ / Vue arienne du réseau polygonal de grandes fentes de gel à colmatage loessique F-4 (cliché D. Gliksman). C/ Hav.1-P1 : détail des déformations en langues de l'unité 5 (gley de toundra) à l'interface entre le loess 4 et le sol 6 (cliché P. Antoine). D/ Hav.2-P9 : sol gris forestier cumulique piégé dans une grande structure de dissolution du substrat crayeux (cliché $\mathrm{P}$. Antoine). E/ Exemple d'échantillonnage en colonne continue à $5 \mathrm{~cm}$ de résolution (Hav.2-P5) (cliché P. Antoine). F/ Hav.2-P9 : colmatage laminé d'une cavité développée à l'interface du remplissage humifère de la dépression et des blocs remaniés du Bt interglaciaire (alternance de silts lavés stratifiés et de lits argilo-humiques) (cliché $\mathrm{P}$. Antoine). G/ Détail du faciès « limon à doublets » des loess décarbonatés directement sous-jacents à l'unité 15 de Bt interglaciaire 15 (cliché P. Antoine). H/ Hav.1-P3 : détail de l'unité 5a/5b de gley de toundra et localisation des tubes de prélèvement pour les datations OSL (cliché P. Antoine). I/ Unité 3 : microstructure lamellaire de gel-dégel et micro-concrétions ferro-manganiques. Lumière polarisée non analysée (LPNA) (cliché S. Coutard). J/ Unité 12 : accumulation de papules (pédoreliques) issues du Bt eemien et probablement regroupées dans une bioturbation. Vides poly-concaves liés à la bioturbation. LPNA (cliché S. Coutard). $\mathrm{K} /$ Unités $14 \mathrm{~b} / \mathrm{c}$ : deux générations d'illuviations argilo-humiques typiques des horizons Bth de sols gris forestiers. LPNA (cliché S. Coutard). 


\subsubsection{2 - Les loess et sols du complexe moyen (unités 6} à 12)

La partie supérieure du complexe de sols (unité 6a) n'est représentée que par le bloc Hav.1-P1-B3. Il s'agit d'un limon quartzeux affecté par une structure lamellaire grossière très développée (espacement $2 \mathrm{~cm}$ ). L'hydromorphie y est soulignée par une ferruginisation de la surface des agrégats, des revêtements dans les vides biologiques et des plages " oxydées » diffuses dans la masse. L'activité biologique y est représentée par de nombreux petits chenaux dont certains contiennent de petits pellets, et quelques cavités biologiques comblées par des gros agrégats arrondis coalescents ou en entassement plus lâche (vers de terre).

L'unité 7 correspond à l'échantillon Hav.1-P1-B2. Il s'agit également d'un limon quartzeux loessique. Un grain de silex sub-anguleux de $3 \mathrm{~mm}$ de longueur a été observé. La masse basale est poussiéreuse et décarbonatée. Des imprégnations noirâtres se sont déposées sur certaines fentes et dans de petits vides biologiques, associées dans quelques cas à des débris de matière organique fibreux. Elles sont postérieures à la structure de gel-dégel. Cette dernière est constituée par une structure lamellaire à espacement millimétrique. Dans la partie inférieure de la lame, les fentes inter-agrégats sont souvent comblées par des particules limoneuses. De gros agrégats arrondis (diamètre atteignant parfois $2,5 \mathrm{~mm}$ ) pourraient témoigner de phases de colluvionnement. L'activité biologique se marque par des cavités contenant de petits pellets (vers de terre, insectes).

Les loess de l'unité 8 sous-jacente ont été prélevés dans le profil P5 (blocs Hav.2-P5-B6 et Hav.2-P5-B7). Ils sont constitués par des limons loessiques homogènes carbonatés, contenant de nombreuses micro-concrétions ferro-manganiques. De grandes cavités biologiques ( $\sim 10 \mathrm{~cm}$ de diamètre) sont comblées soit par des sédiments brun-orangé issus des unités sus-jacentes (7), soit par des limons plus clairs. Quelques papules de ferriargilanes brun-orangé témoignent d'apports locaux intégrés au loess. En dehors des grandes cavités, l'activité biologique est peu marquée ; une fine porosité de chenaux est restée vide.

Deux petits gleys (unités 11 et 9) se développent sous ces loess. A la base de la lame Hav.2-P5-7, le petit gley supérieur (unité 9) se caractérise par de nombreux petits ovoïdes de gélifluxion (arrondis, entourés par une pellicule de particules fines).

Le limon intercalé entre les deux gleys (unité 10, lames Hav.2-P6-A1 et 2) est un limon quartzeux apparem- ment décarbonaté. La partie sommitale est affectée par l'hydromorphie liée au gley sus-jacent avec des microconcrétions ferro-manganiques et quelques concrétions concentriques millimétriques. La microstructure est granulaire. Des cavités biologiques dans lesquelles s'entassent des agrégats coalescents sont visibles.

Le petit gley de l'unité 11 (lames Hav.2-P6-B1) est également nettement hydromorphe : des concrétions ferro-manganiques concentriques sont en place et une ferruginisation de certains agrégats et plages de sédiment se manifeste. La microstructure est granulaire à lenticulaire, avec réarrangement d'agrégats probablement d'origine pédologique ou colluviale (gros agrégats sub-arrondis), qui sont recouverts de fines coiffes sur le dessus. La porosité de chenaux est postérieure à la structure. Des petits débris fibreux de type matière végétale (taille 250-300 $\mu \mathrm{m}$ ) sont assez fréquents.

\subsubsection{3 - Les limons bruns de l'unité 12}

L'unité 12 (limons bruns) qui contient les niveaux archéologiques Hav.1-N1 et Hav.1-N3 a été observée dans plusieurs lames : Hav.2-P6-B2 et C, Hav.2-P8-PGM, Hav.1-P1-1 et Hav.2 P9(GP)-B1 (fig. 4, 7 et 9).

Dans le profil Hav.2-P6, cet horizon est constitué d'un limon quartzeux homogène assez massif. La masse basale, brune, poussiéreuse, est non carbonatée. L'activité biologique est importante : logettes avec accumulation de gros agrégats pluri-millimétriques arrondis (certains bien compactés), petits chenaux verticaux sans doute postérieurs contenant de petits pellets attribuables aux enchytréides. La partie profonde, en Hav.2-P6-C, est légèrement plus massive, mais on $\mathrm{y}$ discerne tout de même des agrégats dans des cavités biologiques. Par ailleurs, Hav.2-P6-C n'est pas affecté par une structure cryogénique, une structure lenticulaire ne se développant que vers le sommet de Hav.2-P6-C et dans Hav.2-P6-B2. Dans l'échantillon PGM (Hav.2-P8), de nombreuses papules de ferri-argilanes roulées issues de l'érosion d'un $\mathrm{Bt}$ et pour certaines d'un Bth sont intégrées au limon, témoignant d'apports locaux importants (fig. 11-J). Certaines papules pourraient s'être accumulées dans des bioturbations. L'activité biologique est intense : chenaux avec comblement de petits pellets arrondis, cavités biologiques plus grandes avec agrégats entassés, aspect parfois grumeleux (fig. 11-J).

Dans la lame Hav.2-P4(GP)-1, il s'agit d'un limon quartzeux à matrice poussiéreuse brunâtre contenant des grains de sables et quelques rares petites papules d'argilanes. Quelques grandes cavités allongées sont comblées

Fig. 11: Hav.2 - Stratigraphy, sedimentology and periglacial features and micromorphology (see table 1 for the description of the units).

A/ Hav.2-P5 : large ice-wedge cast with loess infilling (main level F-4) penetrating the "Havrincourt soil complex" (photo. P. Antoine). B/ Aerial view of the large plolygonal network of ice-wedge casts with loess infilling F-4 (photo. D. Gliksman). C/ Hav.1-P1: detailed view of tongue-like periglacial deformations of unit 5 (tundra gley) between loess 4 and soil 6 (photo. P. Antoine). D/ Hav.2-P9: cumulic forest grey soil trapped in a large dissolution structure of the chalky substrate (photo. P. Antoine). E/ Example of Continuous Sampling Column with a resolution of $5 \mathrm{~cm}$ (Hav.2-P5) (photo P. Antoine). F/ Hav.2-P9: laminated infilling of a cavity developed at the boundary between the humic infilling of the depression and the reworked interglacial Bt blocks (alternation of stratified bleached silts and of clayey humic layers) (photo. P. Antoine). G/ Detailed view of the "limon à doublets" facies of the decalcified loess directly underlying the interglacial Bt horizon unit 15 (photo P. Antoine). H/ Hav.1-P3: detailed view of the tundra gley unit 5a/5b and location of the OSL sampling tubes (photo P. Antoine). I/ Unit 3: freeze-thaw lamellar microstructure and FeMn micro-concretions. Unanalyzed polarized light (photo. S. Coutard). J/ Unité 12: accumulation of clay coating fragments (pedo-relics) reworked from the interglacial Bt horizon and likely concentrated in a biological void. Poly-concave voids resulting from bioturbation. Unanalyzed polarized light (photo. S. Coutard). K/ Unités $14 b / c$ : two generations of stratified clayey humic coatings typical of forest grey soil Bth horizons. Unanalyzed polarized light (photo. S. Coutard). 
par des agrégats ovoïdes à arrondis entassés. Des concrétions ferro-manganiques arrondies $(500 \mu \mathrm{m}-1 \mathrm{~mm})$ sont probablement remaniées. De nombreuses micro-concrétions de diamètre $50 \mu \mathrm{m}$ et des débris fibreux brunâtres inférieurs à $200 \mu \mathrm{m}$ sont dispersés dans la lame.

Dans Hav.1-P1-1, quelques papules de ferri-argilanes sont également présentes (parfois dans des bioturbations), ainsi que quelques pédoreliques et des microconcrétions ferro-manganiques. L'aspect est massif, comme dans Hav.2-P6-C. De grandes cavités biologiques sont comblées par des agrégats arrondis, parfois compactés.

En conclusion, l'unité 12 est caractérisée par une brunification et une activité biologique importante. Le sédiment reste relativement massif, l'agrégation et la bioturbation semblant être plus marquées dans la partie supérieure. Les caractéristiques de cette unité permettent de l'attribuer à un petit sol brun boréal développé sur un limon loessique d'origine partiellement colluviale.

\subsubsection{4 - Le complexe de sols humifères (unités 14a à 14c)}

Les sols humifères ont été échantillonnés localement en 2010 (Hav.1-P4 et P5) et de manière plus systématique en 2011 dans une grande structure (Hav.2-P9, nommé GP pour « grande poche » sur le secteur 2, fig. 9).

Cette « grande poche », liée à la dissolution du substratum crayeux, est comblée par des sédiments brunnoir à dominante humifère, mélangés dans la partie inférieure à des éléments remaniés du Bt 15 (blocs et nodules de sol).

Dans la lame Hav.2-GP-B2 une très grande abondance de papules et de fragments de ferri-argilanes acajou à brun foncé, souvent microlités, est caractéristique du démantèlement d'un horizon Bth de sol gris forestier. Des taches plus claires dans le sédiment correspondent à des cavités biologiques comblées par des limons emballant des papules de ferri-argilanes orangées de type Bt. Dans la lame Hav.2-GP-B3 se retrouvent ces papules. Le sédiment est relativement moins perturbé puisque de petites illuviations argilo-humiques noirâtres sont encore en place au cœur de certains agrégats. La structure est grossièrement lamellaire, les agrégats de grande taille étant basculés avec des coiffes sur le dessus. Les espaces inter-agrégats sont généralement colmatés par des silts blancs lavés.

Plus bas dans la séquence, la lame Hav.2-GP-B4 montre des illuviations argilo-humiques acajou à noirâtres microlitées dans des petits chenaux fragmentés sur place dans les agrégats. Fragments et papules sont dispersés dans les espaces inter-agrégats. La structure est grossièrement lamellaire.

Au niveau de l'échantillon Hav.2-GP-B5 (fig. 9), la structure lamellaire est moins développée. Le sédiment est assez massif. Les illuviations argilo-humiques acajou à noirâtres microlitées sont abondantes dans une fine porosité de chenaux et sont plus ou moins fragmentées sur place. Quelques fragments anguleux sont intégrés à la masse. Quelques concrétions ferro-manganiques arrondies sont dispersées dans la lame. De même, dans l'échantillon de base B8, les ferri-argilanes acajou à noirâtres microlités sont abondants dans la porosité de chenaux. Ils sont en place, mais souvent légèrement fracturés (fig. 11-K). Quelques ferri-argilanes de type Bt brun orangé clair sont présents dans une génération de chenaux antérieurs épars dans la lame. Une grosse pédorelique orangée est également présente. Ainsi, dans la séquence Hav.2-GP, les échantillons issus des blocs B8 à $\mathrm{B} 5$ correspondent à un horizon Bth en place, tandis que les échantillons issus des blocs B4 à B2 témoignent d'un Bth complètement déstructuré puis franchement démantelé par les processus de gel-dégel (coiffes, structure lamellaire).

\subsubsection{5 - Le sol brun lessivé basal (unité 15)}

Le sol interglaciaire peut être observé à travers les reliques présentes dans les lames prélevées dans Hav.2-P9/GP (en particulier Hav.2-P9-B6), et dans trois échantillons provenant du profil Hav.2-P6 (D, E et F). Ces derniers correspondent à un horizon de Bt profond.

Les lames Hav.2-P6-D2 et E1 permettent en effet d'observer quelques illuviations de type ferri-argilanes brun-orangé dans des vides inter-agrégats (en remplissage de cavités biologiques). Le sol est développé sur un limon loessique décarbonaté. La porosité de chenaux est abondante mais généralement vide. L'activité biologique se marque également par des chenaux et cavités remplis d'agrégats arrondis (activité des vers de terre).

\subsubsection{6 - Les loess de base (unité 16)}

L'unité 16 est représentée par la lame Hav.2-P7-1 (fig. 7). La lame mince, haute de $80 \mathrm{~mm}$, est divisible en trois parties : une partie supérieure (0 à $37 \mathrm{~mm}$ ) subdivisée en trois bandes d'une épaisseur de 2,5 à $7 \mathrm{~mm}$, une partie médiane $(37-65 \mathrm{~mm})$ subdivisée en dix-huit bandes parallèles d'une épaisseur de 1 à $2 \mathrm{~mm}$ et une partie inférieure $(65-80 \mathrm{~mm})$ composée d'environ huit bandes parallèles d'une épaisseur de 1 à $2,5 \mathrm{~mm}$.

Le sédiment montre donc à l'œil nu une fabrique plus ou moins bien exprimée en bandes (banded pattern), des bandes riches en fraction fine alternant avec des passées limoneuses loessiques (quartz anguleux bien classés et micas) beaucoup plus propres (fig. 11-G). Très régulières, parallèles et horizontales dans la partie médiane, les bandes présentent un fort pendage dans la partie inférieure. Ceci pourrait traduire une structure sédimentaire préexistante à la formation des doublets.

La limite supérieure des bandes est nette, souvent soulignée par une fissure. Les bandes montrent une distribution de type porphyrique. La fraction fine disparaît progressivement vers le bas. Le sédiment entre les bandes est plus « chaotique » avec des ensembles à distribution chitonique ou mosaïque (action cryogénique ?).

La microstructure est polyédrique à lenticulaire. Les agrégats présentent une microstructure interne en chenaux ou vésiculaire. Les vides et fissures sont orientés préférentiellement parallèlement aux surfaces des bandes. De fines illuviations sont présentes dans quelques chenaux à 
l'intérieur des bandes (revêtement argileux en croissant, limpides à légèrement poussiéreux, souvent microlités dans la partie médiane de la lame).

\section{3 - SÉDIMENTOLOGIE (P.A., G.J.)}

Les analyses effectuées sont les suivantes :

- 1) la granulométrie : méthode de la granulométrie laser en voie humide (Beckman Coulter LS-230). Les échantillons ont d'abord subi une dispersion-défloculation à l'aide d'hexametaphosphate de sodium puis agitation mécanique (pendant $2 \mathrm{~h}$ ). Une coupure par tamisage à $160 \mu \mathrm{m}$ est ensuite effectuée afin d'éliminer les particules grossières non sédimentaires comme les concrétions de $\mathrm{CaCO}_{3}$ secondaire (tubules racinaires notamment) et ferro-manganiques (cf. Antoine et al. (2009) pour le détail du protocole de préparation et d'analyse). La limite des classes est définie par interpolation : argiles $<6 \mu \mathrm{m}$; limons fins $6-20 \mu \mathrm{m}$; limons grossiers 20-61 $\mu \mathrm{m}$ et sables fins 61-160 $\mu \mathrm{m}$ et par comparaison (calibration) avec une série d'échantillons de référence analysée par la méthode conventionnelle (tamisage-pipette; Jamet, 2011);

- 2) la calcimétrie et le Carbone Organique Total (COT) : analyse par combustion sèche à l'aide d'un microanalyseur $\mathrm{CHN}$ (combustion totale du prélèvement à $1050^{\circ} \mathrm{C}$ sous courant d'hélium/oxygène et sous pression / NF ISO 10694 : 1995);

- 3) la susceptibilité magnétique de masse $\left(10 \mathrm{~cm}^{3} /\right.$ échantillon) : mesure à l'aide d'un Bartington MS2B, après séchage à $45^{\circ} \mathrm{C}$, broyage et tamisage à $2 \mathrm{~mm}$ (huit mesures par échantillon avec correction $\pm 1 \sigma$ ).

Afin d'éviter les redondances, la discussion détaillée des différents paramètres analytiques illustrés par les figures 12 à 14 est intégrée à la synthèse (cf. partie 4). Le texte ci-dessous se contente de décrire les grandes lignes de la variation des différents paramètres en fonction de la stratigraphie synthétique (fig. 12-14).

D'une manière générale, il est noté pour toutes les séries analytiques une très bonne corrélation avec les limites stratigraphiques identifiées sur le terrain. Ces transitions s'effectuant parfois d'un échantillon à un autre donc sur moins de cinq centimètres. Par ailleurs les gammes de valeurs sont très proches de celles obtenues sur les séquences de références régionales, analysées par d'autres laboratoires (INRA Arras) et/ou avec des méthodologies différentes, notamment pour la granulométrie (pipette et tamisage). Ces observations nous permettent d'affirmer la reproductibilité et la fiabilité des données analytiques produites pour les séquences d'Havrincourt.

Tout d'abord, les signaux granulométriques permettent de mettre en évidence un contraste très net entre les unités purement loessiques caractérisées par des taux d'argile de 12 à $14 \%$ maximum et les niveaux du complexe de sol des unités 7-6 atteignant 25 à $29 \%$. La partie supérieure du Bt de surface de l'unité 1a, ainsi que l'horizon Bt 15 affichent par contre des valeurs assez faibles $(\sim 22 \%)$ pour ce type d'horizon, ce qui semble confirmer leur caractère fortement tronqué (Bt profond). Cette érosion semble aussi indiquée par l'interruption au niveau de la surface de la progression régulière des valeurs observées dans cet horizon à partir de $70 \mathrm{~cm}$ de profondeur. Enfin, on remarquera que l'horizon brun de l'unité 12 ne montre pas d'enrichissement en argile (max. $20 \%$ ) par rapport au loess sus-jacent des unités 11 à 8 .

Toujours dans le registre granulométrique, l'indicateur IGR représentant le rapport entre les fractions limons grossiers et limons fins ajoutés aux argiles (ou le U-ratio excluant les argiles) permet de bien mettre en évidence les grandes phases de dépôts loessiques qui se distinguent par des valeurs nettement plus élevées et toujours supérieures à 1 (Antoine et al., 2009).

A Havrincourt, les variations de l'IGR permettent de décrire deux grandes phases de dépôt loessique à IGR élevé $(>1)$ dans les unités $1 \mathrm{~b}$ à $1 \mathrm{e}$ (malgré leur décarbonatation) et 4 . Plus bas on retrouve des valeurs de 1 à 1,4 dans la partie supérieure du loess de l'unité 8 et de 1 à 1,2 dans l'ensemble des unités 9 à 12 . Il est frappant de noter que la structure de ces deux pics d'IGR enregistrés à Havrincourt sur les 3,3 m supérieurs, se retrouve dans les 4 m supérieurs de la coupe de Beugnâtre située à $14 \mathrm{~km}$ à l'ouest (fig. 15).

Le signal du carbone organique apparaît lui aussi très bien corrélé à la stratigraphie (fig. 14). Il souligne clairement les différents épisodes de pédogenèse, notamment ceux du complexe de sols constitué par les unités 6 et 7 , qui se caractérise par un doublement de la concentration par rapport à celle des loess (de $0,16-0,18 \%$ à $0,35-0,45 \%$ ). On remarque aussi que la bipartition du gley de toundra complexe de l'unité $3 a-3 c$ est très bien marquée par des valeurs plus élevées (surtout en 3c : 0,24-0,26\%) que celles du niveau loessique intermédiaire ( $3 \mathrm{~b}: 0,17 \%$ ). Il apparaît par ailleurs que le loess parent du sol de surface (unité 2) n'est affecté par une augmentation progressive des concentrations de COT qu'à partir de $-1,25 \mathrm{~m}$ sous la surface actuelle (surface agricole cultivée et enrichie en engrais et matière organique). Au-dessous de cette limite, le loess, bien que décarbonaté par lessivage, montre des concentrations très basses identiques à celle des loess calcaires sous-jacents $(\sim 0,12-0,16 \%)$. Cette observation est importante car elle permet de supposer que les ossements associés au niveau archéologique Hav.2-N2 et datés par radiocarbone n'ont pas subi de pollution par du carbone organique récent.

D’une manière générale, les valeurs maximales $(0,5 \%)$ apparaissent dans la partie supérieure du complexe de sol humifère (unité 14) et les valeurs de COT mesurées dans les différents horizons d'Havrincourt sont parfaitement cohérentes avec celles obtenues dans les autres séquences régionales comme Saint-Sauflieu (Antoine et al., 1994), Bettencourt (Antoine et al., 2003a), Villiers-Adam (Locht et al., 2003) ou Beugnâtre (Antoine et al., 2005).

Enfin, la susceptibilité magnétique, dont les valeurs absolues s'intègrent dans la gamme habituelle pour ce type d'environnement, ne montre par contre pas de très forts contrastes notamment en ce qui concerne le complexe de sols des unités 7-6. 


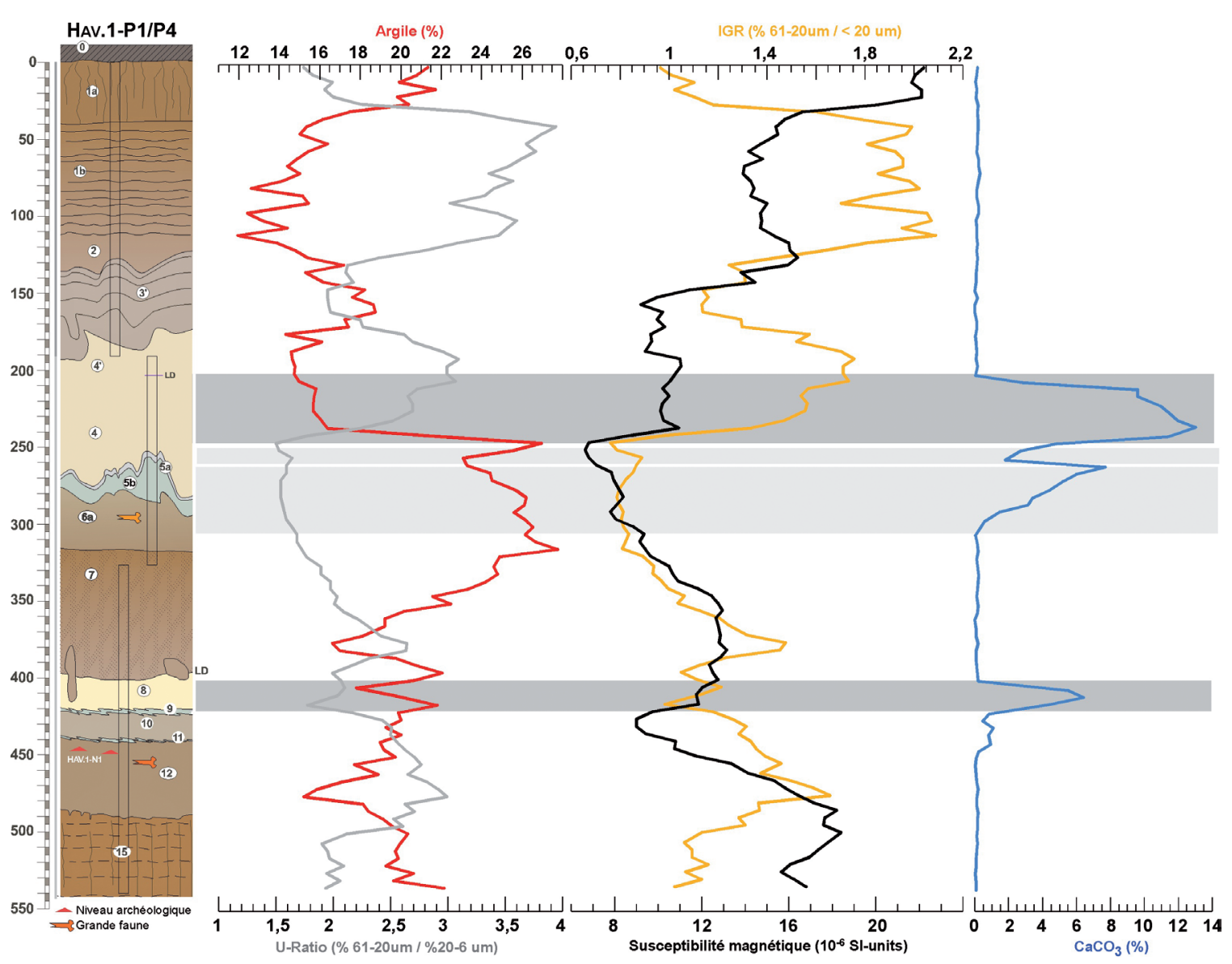

Fig. 12 : Hav.1 - profil synthétique P1-P4 : données sédimentologiques.

Fig. 12: Hav.1 - synthetic profile P1-P4: sedimentological data.

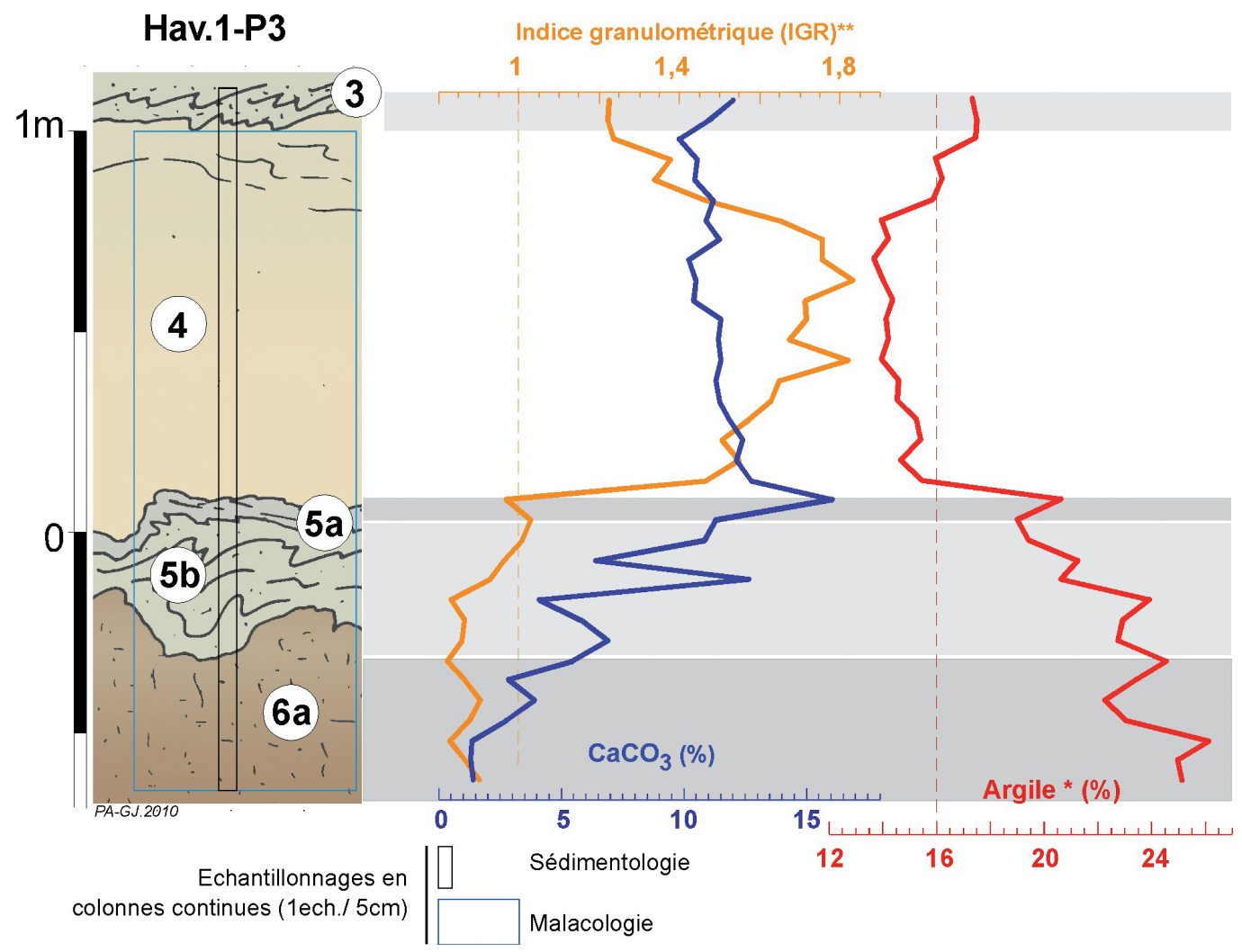

Fig. 13 : Hav.1 - profil P3 : données sédimentologiques.

Fig. 13: Hav. 1 profile P3: sedimentological data. 


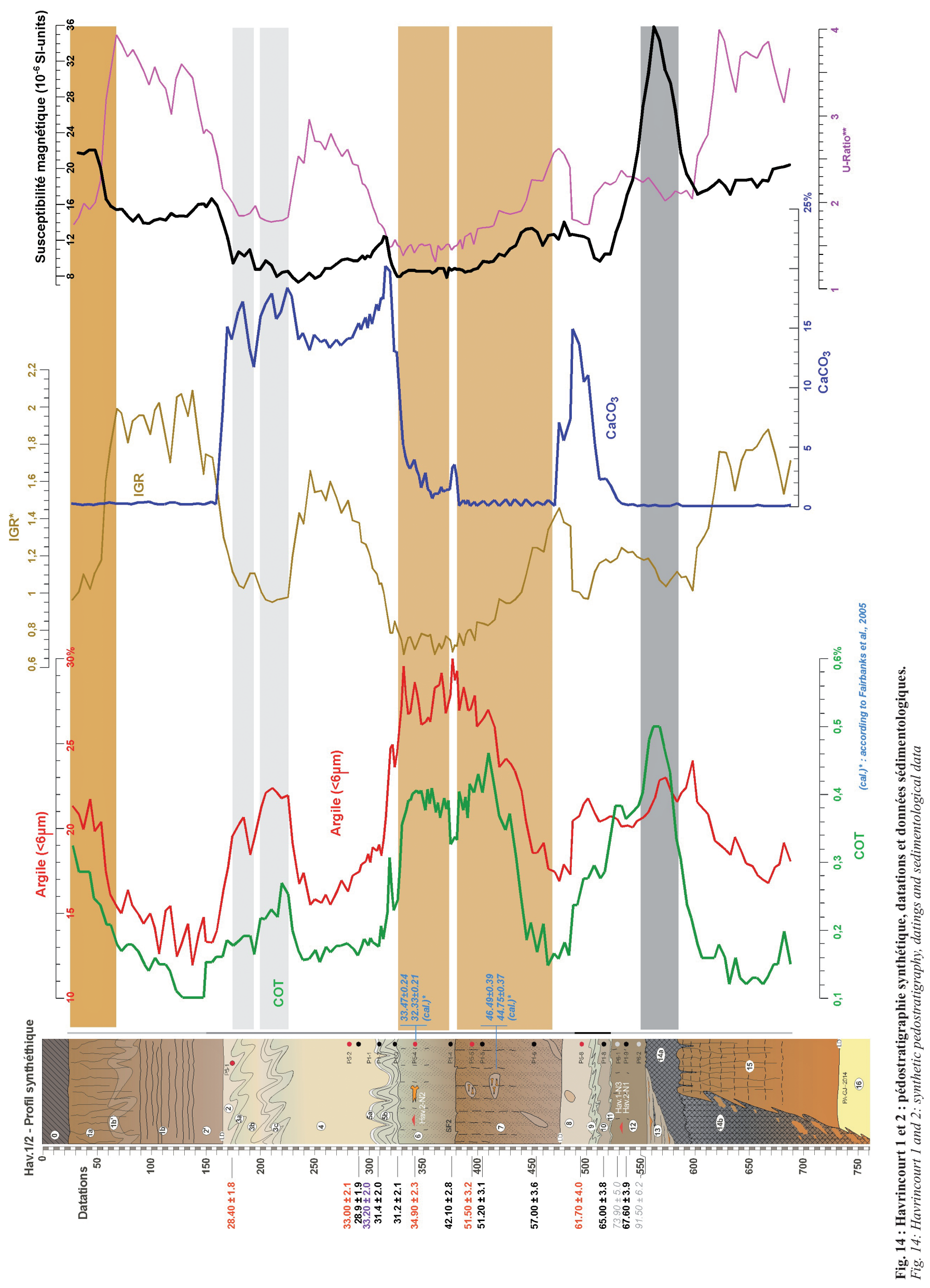


Les valeurs maximales sont classiquement observées dans l'horizon humifère de l'unité 14 (36 SI units), ainsi que dans les $20 \mathrm{~cm}$ de l'horizon de labour et les $30 \mathrm{~cm}$ supérieurs de l'horizon Bt du sol de surface (18 à 23 SI units) mais restent assez faibles en valeur absolue par rapport à d'autres sites régionaux (horizons fortement tronqués). A l'opposé, les valeurs les plus basses sont associées aux loess calcaires (unités 8-10) et aux gleys de toundra comme l'unité 3 (dégradation des minéraux ferromagnétiques dans les gleys par les processus d'hydromorphie). Le gley de toundra de l'unité 5a montre cependant un pic assez net qui est associé au petit horizon grisâtre d'apparence encroûtée de 3 à $4 \mathrm{~cm}$ d'épaisseur où d'ailleurs les valeurs de $\mathrm{COT}$ et de $\mathrm{CaCO}_{3}$ remontent brutalement (micro-sol de toundra ?).

D'une manière un peu surprenante, le complexe de sols des unités 7-6 ne montre aucune augmentation notable des valeurs de susceptibilité, seule une petite élévation apparaît dans les $45 \mathrm{~cm}$ de la base de l'unité 7 . Il est donc clair que ces horizons bruns enrichis en argile résultent d'un type de pédogenèse bien différent de celui des Bt interglaciaires ou des horizons humifères du Début Glaciaire.
Enfin l'horizon brun 12 montre même des valeurs nettement décroissantes, de $\sim 20$ à la base à $\sim 10$ SI units au sommet, qui signent une évolution inverse de celle généralement caractéristique d'un sol en place. Cette observation renforce l'interprétation colluviale du mode de dépôt de cette unité proposé préalablement. Cet horizon brun grisâtre aurait été alimenté, notamment dans sa moitié inférieure, par l'érosion et le remaniement par ruissellement d'horizons de sols à fortes valeurs de susceptibilité magnétique comme l'horizon organique de l'unité 14 ou le sommet du Bt de l'unité 15, en accord avec les observations micromorphologiques (présence de nodules de sol, pédoreliques).

Enfin, sans surprise, les valeurs de $\mathrm{CaCO}_{3}$ montrent de très fortes variations entre les faciès de loess calcaires comme l'unité 4 (15-18\%) ou le gley de toundra carbonaté (unité 3) et les faciès de sol brun de l'unité 7 totalement décarbonatés. Il est intéressant de noter la présence d'un pic de $\mathrm{CaCO}_{3}$ à la base de l'unité 6 juste au-dessus de la transition avec l'unité 7 ainsi qu'une tendance à l'enrichissement progressif des valeurs à travers les unités 6 et la transition avec l'unité 5 .

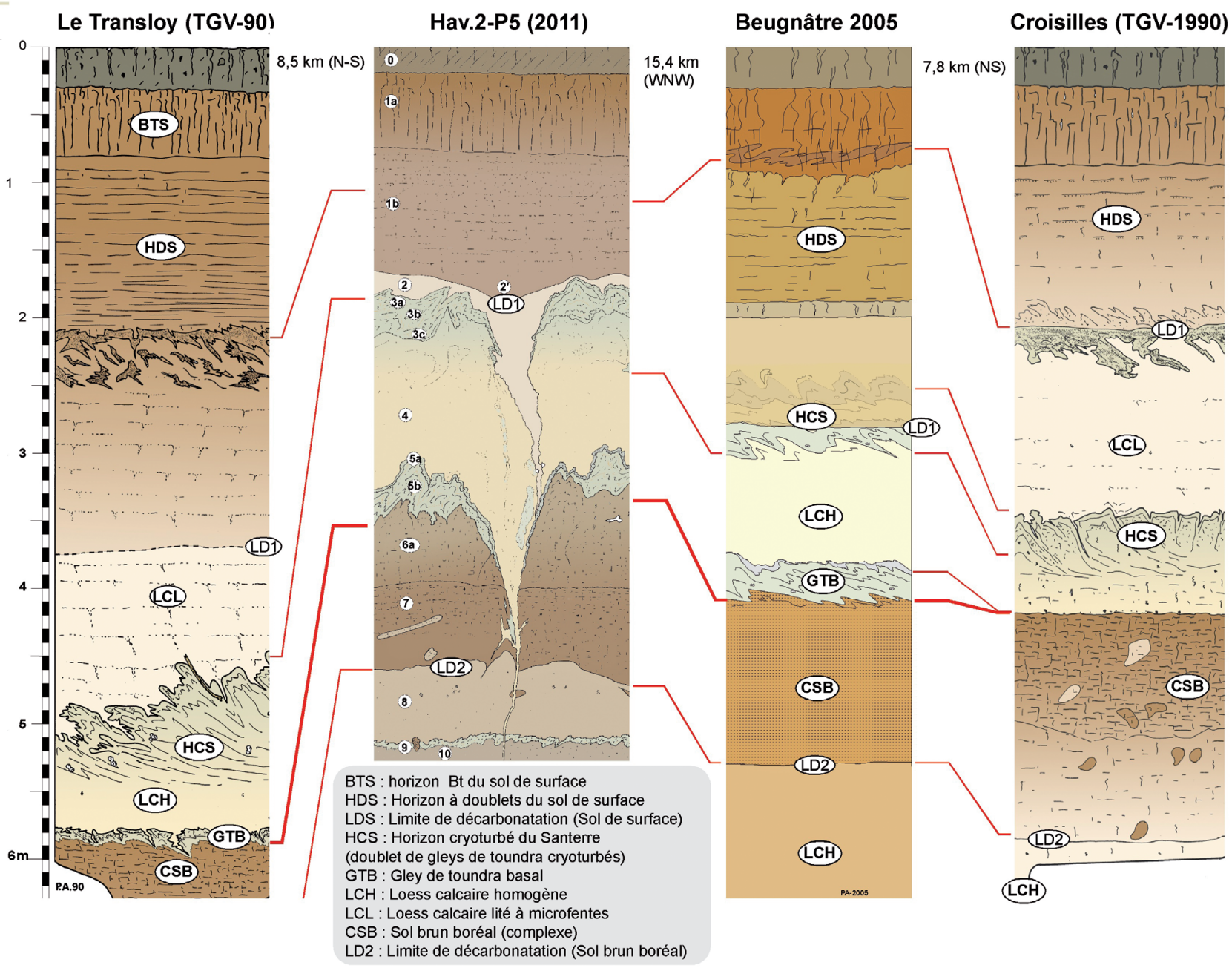

Fig. 15 : Corrélations entre la séquence d'Havrincourt (Hav.2-P5) et quelques profils pédosédimentaires régionaux (profils du Transloy et de Croisilles d'après Antoine (1991) modifié, et de Beugnâtre d'après Antoine (2005)).

Fig. 15: Correlation between the Havrincourt profile (Hav.2-P5) and some regional pedosedimentary sequences (profiles from Le Transloy and Croisilles according to Antoine (1991) modified, and from Beugnâtre according to Antoine (2005)). 


\subsection{1 - Anisotropie de la susceptibilité magnétique (ASM) (F.L.)}

L'anisotropie de la susceptibilité magnétique (ASM) est définie par l'orientation cristallographique ou dimensionnelle préférentielle de tous les minéraux. Nous parlons d'une fabrique magnétique. Dans le cas de sédiments loessiques, la fabrique magnétique peut indiquer une sédimentation primaire éolienne avec ou sans empreinte des directions de vent dominant ou d'un remaniement survenant après la sédimentation éolienne.

La susceptibilité magnétique ( $\kappa)$ est mesurée sur trois plans orthogonaux avec un Kappabridge (KLY-3) définissant un tenseur de second ordre, que nous pouvons visualiser par une ellipsoïde. L'ellipsoïde de l'ASM est caractérisée par trois axes principaux orthogonaux dits axe maximal $\left(\kappa_{\mathrm{MAX}}\right)$, axe intermédiaire $\left(\kappa_{\mathrm{INT}}\right)$ et axe minimal $\left(\kappa_{\mathrm{MIN}}\right)$ ayant chacun une grandeur et une direction (déclinaison et inclinaison). L'excentricité de l'ellipsoïde de l'ASM ou le degré d'anisotropie est traduit par le paramètre $\mathrm{P}_{\mathrm{j}}$ et la forme de l'ellipsoïde par le paramètre $T_{j}$ défini ci-dessous suivant Jelínek (1981) :

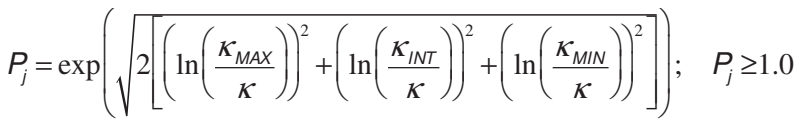

$$
\begin{aligned}
& T_{j}=\frac{\ln \left(\frac{\kappa_{I N T}}{\kappa_{\text {MIN }}}\right)-\ln \left(\frac{\kappa_{M A X}}{\kappa_{I N T}}\right)}{\ln \left(\frac{\kappa_{I N T}}{\kappa_{\text {MIN }}}\right)+\ln \left(\frac{\kappa_{\text {MAX }}}{\kappa_{I N T}}\right)} ; \frac{-1 \leq T_{j} \leq 1}{\text { (prolate) (oblate) }}
\end{aligned}
$$

où $\kappa$ est la moyenne des grandeurs des trois axes principaux. A chacun des dix-neuf intervalles entre 3,05 et 2,05 $\mathrm{cm}$ de profondeur 4 à 6 spécimens ont été mesurés et la moyenne tensorielle calculée suivant Jelínek (1976) d'après Hext (1963) dans le programme PaleoMac (Cogné, 2003).

La susceptibilité magnétique moyenne $\left(\kappa_{\text {moyenne }}\right)$ des spécimens cubiques orientés et de la moyenne tensorielle par intervalles décroît de la base de l'unité 4 à son sommet (fig. 16A). Ceci est conforme aux mesures effectuées sur les échantillons non orientés (fig. 14).

L'ellipsoïde de l'ASM des spécimens ainsi que des moyennes tensorielles est de forme oblate avec des valeurs moyennes de $\mathrm{T}_{\mathrm{j}}$ de $0,64 \pm 0,21$ et $0,75 \pm 0,16$ et un degré

Fig. 16 : Résultats d'anisotropie magnétique (ASM) pour l'unité 4 du profil Hav.2-P5.

A/ Susceptibilité magnétique moyenne $(\kappa$,$) des trois principaux$ axes $\left(\kappa_{\mathrm{MAX}}, \kappa_{\mathrm{INT}}\right.$, et $\left.\kappa_{\mathrm{MIN}}\right)$ de l'ellipsoïde de l'ASM pour chaque spécimen (ronds noirs), $\mathrm{n}=104$, et moyenne tensorielle des spécimens à un intervalle de profondeur (croix rouges). B/ Degré d'anisotropie de l'ellipsö̈de de l'ASM en fonction de la profondeur. C/ Variation de la déclinaison de $\kappa_{\mathrm{MAX}}$ en fonction de la profondeur, l'angle d'incertitude de la déclinaison dans le plan de la foliation $\left(\varepsilon_{12}\right)$ est représenté pour la moyenne tensorielle uniquement. $\mathrm{D} /$ Projection stéréographique sur l'hémisphère du bas de l'orientation des principaux axes $\left(\kappa_{\mathrm{MAX}}=\right.$ carrés rouges, $\kappa_{\mathrm{INT}}=$ triangles bleus, et $\kappa_{\text {MIN }}=$ ronds verts) de l'ellipsoïde de l'ASM.

Fig. 16: Anisotropy of magnetic susceptibility (AMS) results of unit 4 of the Hav.2-P5 profile. A/ Variation of the mean magnetic susceptibility $\left(\kappa_{\text {movenne }}\right)$ of the three main axes $\left(\kappa_{M A X}, \kappa_{I N T}\right.$ and $\left.\kappa_{M I N}\right)$ of the AMS ellipsoid of individual specimens (black circles), $n=104$, and of the depth interval tensor mean (red crosses) with depth. B/Variation of the degree of anisotropy of the AMS ellipsoid with depth. C/ Variation of $\kappa_{M A X}$ declination with depth, the angle of uncertainty around $\kappa_{M A X}$ within the foliation plane $\left(\varepsilon_{1}\right)$ is shown for the depth interval tensor means only. $D /$ Lower hemisphere stereographic projection of the AMS principal axes $\left(\kappa_{M A X}=\right.$ red squares, $\kappa_{I N T}=$ blue triangles, and $\kappa_{M I N}=$ green circles). d'anisotropie $P_{j}$ moyen de respectivement 1,03 $\pm 0,01$ et $1,03 \pm 0,03$. L'unité 4 ne montre aucune variation systématique de $\mathrm{Tj}$ en fonction de la profondeur ; par contre nous observons une augmentation de $\mathrm{Pj}$ de 3,05 m à 2,65 m suivi d'une décroissance jusqu'à 2,25 m (fig. 16B). La distribution spatiale des axes principaux de l'ASM (fig. 16D) définit une fabrique oblate, caractéristique d'une fabrique

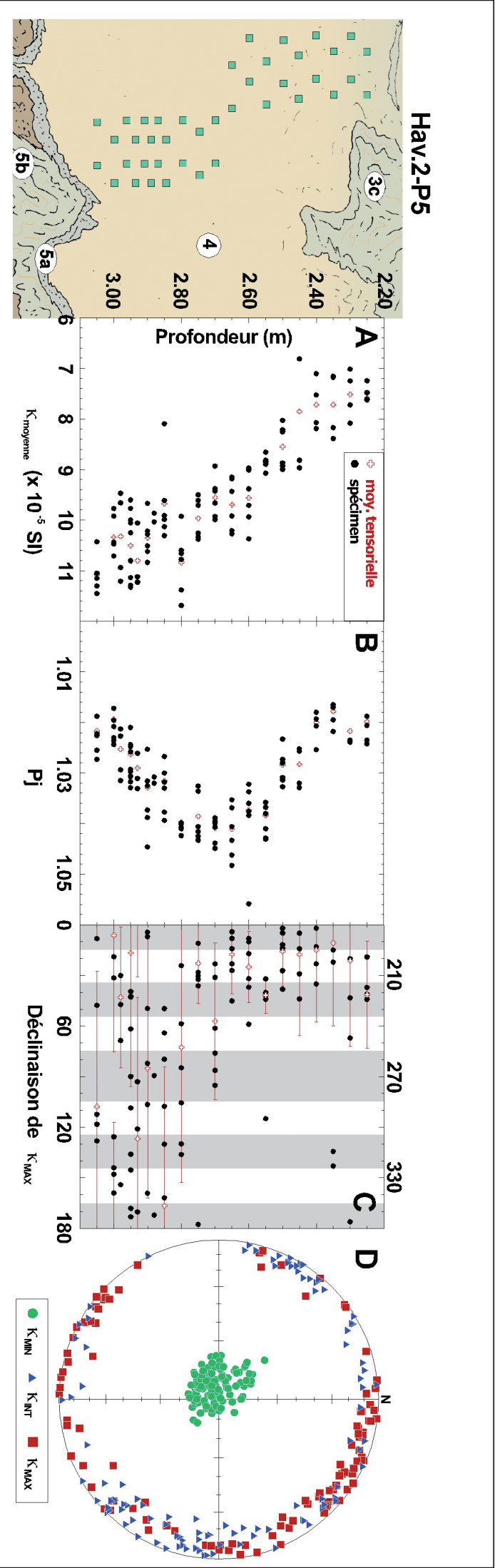


sédimentaire, ou $\kappa_{\mathrm{MIN}}$ représente le pôle de la foliation magnétique et $\kappa_{\mathrm{INT}}$ et $\kappa_{\mathrm{MAX}}$ reposent dans le plan de la foliation. Les axes $\kappa_{\text {MIN }}$ sont resserrés autour d'une orientation moyenne de 255/83 correspondant à une surface de dépôts quasi-horizontale ayant un léger pendage vers le nord-est comme la topographie actuelle. Sur la projection stéréographique (fig. 16D), les axes $\kappa_{\mathrm{INT}}$ et $\kappa_{\mathrm{MAX}}$ sont dispersés le long du plan de la foliation magnétique et ne permet pas de déceler une orientation préférentielle de $\kappa_{\mathrm{MAX}}$ correspondant à une linéation magnétique. Ceci dit, une analyse des variations de la déclinaison de $\kappa_{\text {MAX }}$ de la base de l'unité 4 à son sommet montre que celle-ci est mal contrainte de $3,05 \mathrm{~m}$ à $2,80 \mathrm{~m}$. A une même profondeur, la déclinaison est très variable ce qui se traduit par un angle d'incertitude dans le plan de foliation $\left(\varepsilon_{12}\right)$ des déclinaisons de $\kappa_{\mathrm{MAX}}$ de la moyenne tensorielle sur cet intervalle de $74 \pm 14$ degrés. Autrement dit, dans le plan de la foliation, la susceptibilité magnétique est isotrope. Entre 2,75 m et 2,25 m, les déclinaisons de $\kappa_{\mathrm{MAX}}$ sont mieux contraintes. La reproductibilité de la déclinaison de $\kappa_{\mathrm{MAX}}$ des spécimens à une profondeur donnée est supérieure et se traduit par un $\varepsilon_{12}$ moyen de $33 \pm 14$ degrés sur cet intervalle. Une linéation magnétique, bien que faible, semble être superposée à la foliation magnétique et celle-ci à une orientation préférentielle NNE / SSO. Dans les milieux loessiques de l'Alaska, il a été montré qu'une linéation magnétique significative mais faible, de l'ordre de 15 à $20 \%$ de l'anisotropie totale, était représentative de la direction des vents dominants au moment de la sédimentation éolienne (Lagroix \& Banerjee, 2002, 2004). Sur cette base, les résultats obtenus dans l'unité 4 peuvent être interprétés en termes de directions de vent au moment du dépôt.

L'IGR et le U-ratio (fig. 12 et 14) identifient l'unité 4 comme une phase de forte sédimentation loessique. Les valeurs maximales de ces indices sont observées entre 2,75 $\mathrm{m}$ et 2,45 $\mathrm{m}$ en correspondance avec l'intervalle ou la linéation magnétique est la mieux contrainte. De plus, à la base et au sommet de l'unité 4, l'IGR et l'U-ratio sont plus faibles, comme le degré d'anisotropie. L'intensification du transport éolien suggérée par l'IGR et l'U-ratio est vraisemblablement accompagnée d'une augmentation des vitesses moyennes des vents permettant le développement d'une linéation magnétique. Celle-ci nous indique une direction dominante des vents orientés dans un axe NNE / SSO.

\section{4 - DATATION}

\subsection{1 - OSL-quartz (G.G., P.A., E.S.)}

\subsubsection{1 - Méthodologie}

Il était important de définir une stratégie en amont de l'échantillonnage selon le type d'étude à réaliser (géomorphologique, géologique, archéologique) mais aussi du caractère approprié et de l'aptitude du matériel pour la datation par OSL. Les loess sont bien adaptés à la datation par OSL car leur signal de luminescence géologique est blanchi par la lumière lors de leur transport éolien. Toutefois, pour que la paléodose puisse être correctement interprétée, il faut connaître la profondeur de l'enfouissement de l'échantillon et prêter attention à l'évolution post-dépositionnelle du sédiment, à l'altération diagénétique et pédologique. De plus, il faut estimer les possibles variations de l'humidité du sédiment et vérifier l'homogénéité lithologique de la zone de prélèvement (sur un rayon d'au moins $30 \mathrm{~cm}$ à cause de la portée des rayons gamma). C'est pour cela que les prélèvements ne sont faits que dans les zones les plus appropriées.

Sur les deux sites d'Havrincourt, Hav.1 et Hav.2, 23 échantillons ont ainsi été prélevés sans exposition à la lumière à l'aide de tubes en cuivre de 35 x $140 \mathrm{~mm}$. Ces tubes ont été introduits horizontalement dans le sédiment avec un marteau puis scellés aux deux extrémités avec une bande adhésive. Pour la détermination de la dose annuelle et la teneur en eau, environ $2 \mathrm{~kg}$ de sédiments ont été prélevés de manière à moyenner la composition sur un rayon de $30 \mathrm{~cm}$ autour du point de chaque prélèvement pour l'OSL.

Pour les analyses de luminescence, les opérations de préparation et de mesure ont été réalisées en éclairage contrôlé. Une fraction polyminérale $(4-11 \mu \mathrm{m})$ a été extraite de la partie centrale des tubes d'échantillonnage en utilisant les techniques proposées par Frechen et al. (1996). Les grains de quartz ont été isolés de cette fraction par une attaque de 5 à 12 jours par de l'acide hexafluorosilicique (H2SiF6), suivie d'un rinçage à l'acide chlorhydrique ( $3 \mathrm{~N}, 1 \mathrm{~h})$. Les aliquotes servant aux mesures de luminescence ont été préparées par sédimentation d'une suspension des quartz dans l'acétone $(\sim 1 \mathrm{mg} / \mathrm{ml})$ sur des disques en acier de $10 \mathrm{~mm}$ de diamètre. Le dépôt finalement obtenu sur les disques est d'environ $1 \mathrm{mg} / \mathrm{cm}^{2}$.

Toutes les mesures de luminescence ont été effectuées en utilisant un système Risø TL/OSL DA-15 à 48 positions équipé de diodes bleues (470 nm) et d'une diode laser IR (Bøtter-Jensen et al., 2002). La luminescence optique a été mesurée avec un tube photomultiplicateur Thorn-EMI 9235QA au travers d'un filtre Hoya U340 de 7,5 mm d'épaisseur. La pureté de chaque préparation de quartz a été vérifiée en mesurant la sensibilité à une stimulation infrarouge (IRSL) après une irradiation bêta. Ce signal s'est avéré être en général proche de $1 \%$ et toujours inférieur à $3 \%$ à celui obtenu par stimulation par les diodes bleues (OSL), attestant de l'absence de feldspaths résiduels.

Les irradiations en laboratoire ont été réalisées par la source $90 \mathrm{Sr} / 90 \mathrm{Y}$ du lecteur TL/OSL. Le débit de cette source a été contrôlé et calibré en utilisant une source gamma de débit connu dont dispose le Laboratoire des Sciences du Climat et de l'Environnement. Cette calibration a été faite dans les mêmes conditions de géométrie de mesure que celles des échantillons et pour les mêmes densités de dépôt sur les disques, évitant ainsi d'introduire une erreur systématique (Guérin \& Valladas, 2014).

Tous les échantillons étudiés ont des propriétés OSL comparables. En particulier, tous présentent un signal d'OSL fortement dominé par la composante rapide (Murray \& Wintle, 2003) avec une diminution de plus de $90 \%$ du signal en moins de $1,2 \mathrm{~s}$. Lors de chaque lecture de l'OSL, la stimulation bleue dure $40 \mathrm{~s}$; le signal est 
intégré sur les deux premières secondes de stimulation et le bruit de fond sur l'intervalle 33 à $35 \mathrm{~s}$.

Les paramètres des protocoles du SAR ont été fixés après avoir soumis les échantillons au « test du plateau » qui consiste à examiner l'évolution de la dose apparente déduite du protocole SAR en fonction de la température de préchauffe. Ce test, réalisé pour tous les échantillons par pas de $20^{\circ} \mathrm{C}$ pour des températures de $180^{\circ} \mathrm{C}$ à $320^{\circ} \mathrm{C}$, fournit un plateau acceptable entre $220^{\circ} \mathrm{C}$ et $280^{\circ} \mathrm{C}$. L'examen complémentaire du rapport de recyclage (qui représente l'évolution de la sensibilité entre le premier et le dernier cycle de mesure) et du rapport d'affaiblissement par l'infrarouge (IR depletion ratio) nous ont conduit à choisir pour tous les échantillons les mêmes conditions de mesure : soit une préchauffe de $10 \mathrm{~s}$ à $260^{\circ} \mathrm{C}$ pour le signal de régénération et une préchauffe de $1 \mathrm{~s}$ à $180^{\circ} \mathrm{C}$ pour la dose test (normalisation). Enfin, le dernier critère de qualité consiste, après effacement du signal naturel de l'échantillon à mesurer par le protocole $\mathrm{SAR}$ sa réponse à une dose connue. Ce critère (Dose Recovery Test) proposé par Murray et Wintle (2003), bien qu'il ne soit pas universel
(Wang et al., 2011), a été vérifié pour tous les échantillons et s'est révélé satisfaisant à mieux que $3 \%$ près.

La quantification de la radioactivité naturelle a été faite en mesurant les concentrations en Uranium (U), Thorium (Th) et Potassium (K) par spectrométrie gamma à haute résolution du sédiment prélevé autour du point d'échantillon. Ces mesures ont été réalisées au laboratoire de Modane sur un détecteur germanium haute pureté (GeHP) à très bas bruit de fond et n'ont pas montré de déséquilibre dans les chaînes de décroissance radioactives de l'uranium ou du thorium (tab. 2A). Les différentes composantes du débit annuel de dose (tab. 2B) sont calculées à partir de ces concentrations en utilisant les facteurs de conversion révisés par Guérin et al. (2011). L'incidence sur la dosimétrie de la teneur en eau de la colonne sédimentaire est prise en compte en utilisant les coefficients de saturation en eau mesurés pour chaque échantillon. Le calcul des débits annuels de dose et des incertitudes associées est fait en supposant que la moyenne au cours du temps de la teneur en eau du sédiment se trouve (de manière équiprobable) entre $60 \%$

A

\begin{tabular}{|l|c|c|c|c|c|c|}
\hline & $\mathrm{U}(\mathrm{ppm})$ & \pm & $T h(\mathrm{ppm})$ & \pm & $K(\%)$ & \pm \\
\hline HAV1-P1-1 & 2,89 & 0,40 & 10,62 & 0,27 & 1,27 & 0,05 \\
\hline HAV1-P1-2 & 3,38 & 0,24 & 11,39 & 0,15 & 1,29 & 0,02 \\
\hline HAV1-P1-3 & 4,10 & 0,40 & 11,81 & 0,27 & 1,35 & 0,04 \\
\hline HAV1-P1-4 & 3,31 & 0,40 & 12,92 & 0,27 & 1,37 & 0,05 \\
\hline HAV1-P1-5 & 3,30 & 0,32 & 12,18 & 0,25 & 1,34 & 0,04 \\
\hline HAV1-P1-6 & 3,86 & 0,40 & 11,71 & 0,27 & 1,42 & 0,05 \\
\hline HAV1-P1-7 & 4,14 & 0,19 & 13,23 & 0,13 & 1,65 & 0,02 \\
\hline HAV1-P1-8 & 3,15 & 0,22 & 10,73 & 0,17 & 1,28 & 0,03 \\
\hline HAV1-P1-9 & 3,30 & 0,24 & 12,15 & 0,15 & 1,52 & 0,03 \\
\hline HAV1 P3-10 & 2,67 & 0,27 & 8,68 & 0,15 & 1,22 & 0,01 \\
\hline HAV2-P5-1 & 3,40 & 0,31 & 10,18 & 0,17 & 1,41 & 0,03 \\
\hline HAV2 P5-2 & 3,05 & 0,27 & 8,87 & 0,15 & 1,20 & 0,03 \\
\hline HAV2-P5-4 & 3,70 & 0,40 & 11,98 & 0,27 & 1,37 & 0,04 \\
\hline HAV2 P5-5 & 3,27 & 0,17 & 11,49 & 0,10 & 1,37 & 0,03 \\
\hline HAV2-P5-8 & 3,30 & 0,40 & 10,62 & 0,25 & 1,41 & 0,04 \\
\hline HAV2 P6-1 & 3,37 & 0,31 & 11,49 & 0,17 & 1,58 & 0,03 \\
\hline HAV2 P6-2 & 3,19 & 0,30 & 11,51 & 0,17 & 1,67 & 0,03 \\
\hline
\end{tabular}

B

\begin{tabular}{|c|c|c|c|c|c|c|c|c|c|c|c|c|c|c|c|c|c|}
\hline & $\begin{array}{l}\text { Profondeur } \\
(\mathrm{cm})\end{array}$ & $\begin{array}{l}W \\
(\%)\end{array}$ & \pm & $\begin{array}{c}\text { Paléodose } \\
\text { (Gy) }\end{array}$ & \pm & $\begin{array}{c}\text { Dose } \\
\text { alpha } \\
\mathrm{mGy} / \mathrm{an}\end{array}$ & \pm & $\begin{array}{l}\text { Dose } \\
\text { bêta }\end{array}$ & \pm & $\begin{array}{c}\text { Dose } \\
\text { gamma }\end{array}$ & \pm & $\begin{array}{c}\text { Dose } \\
\text { cosmique }\end{array}$ & \pm & $\begin{array}{l}\text { Dose } \\
\text { totale }\end{array}$ & \pm & $\begin{array}{l}\text { Age } \\
\text { (ka) }\end{array}$ & \pm \\
\hline HAV1-P1-1 & 270 & 35,0 & 3,5 & 80,9 & 1,5 & 0,50 & 0,05 & 28 & 0,0 & 087 & 0,05 & & 0,02 & 2,80 & 0,18 & 28,9 & 1,9 \\
\hline HAV & & 3,9 & 4 & & & & & 28 & 0, & & 75 & & & 2,79 & 0,17 & 31,4 & 2,0 \\
\hline HAV1 & & 2,2 & 4,2 & & 1, & & & 41 & 0, & & 6 & & & 3,12 & 0,20 & 31,2 & 2,1 \\
\hline $\mathrm{HA}$ & & 9,3 & 3,9 & 1 & 2,2 & & 0,05 & 39 & 0, & & & & 1 & 3,01 & 0,19 & 42,1 & 2,8 \\
\hline HAl & & 9,4 & 3,9 & 1 & 2 , & & 0,05 & 35 & 0, & & & & 0,01 & 2,93 & 0,17 & 51,2 & 3,1 \\
\hline $\mathrm{HA}$ & & 38,4 & 3,8 & 1 & 3 & & 0,05 & 46 & $0,($ & & 0,06 & & 0,01 & 3,13 & 0,19 & 57,0 & 3,6 \\
\hline $\mathrm{HA}$ & & 42,9 & 4,3 & 1 & 2, & & 0, & 1,60 & $0,($ & 1 & 0,06 & & 0,01 & 3,39 & 0,19 & 48,7 & 2,9 \\
\hline $\mathrm{HA}$ & 4 & 38,2 & 3,8 & 177,5 & 3,2 & 0 , & 0,04 & 1,29 & $0,($ & 0,8 & 0,05 & & 0,01 & 2,73 & 0,15 & 65,0 & 3,8 \\
\hline $\mathrm{HA}$ & 5 & 41,8 & 4,2 & 202,1 & 2 & 0,49 & 0,04 & 1,43 & $0, \mathrm{C}$ & 0,9 & 0,05 & & 0,01 & 2,99 & 0,17 & 67,6 & 3,9 \\
\hline & & 36,1 & 3,6 & 84, & 1, & & 0,04 & 18 & 0,0 & 0,7 & 0,04 & & 0,02 & 2,53 & 0,14 & 33,2 & 2,0 \\
\hline & & 43,1 & 4,3 & 81 & 2 , & 0, & 0,05 & 1,33 & 0,0 & 0,8 & $0, C$ & & 0,02 & 2,86 & 0,17 & 28,4 & 1,8 \\
\hline $\mathrm{HAl}$ & & 39,0 & 3,9 & 84 & 1, & & 0,04 & 1,19 & 0,07 & 0,7 & 0,0 & & 0,01 & 2,55 & 0,15 & 33,0 & 2,1 \\
\hline HAV2-P5-4 & & 43,8 & 4,4 & 103,6 & 2 , & & 0,05 & 1,37 & 0,09 & 0 & 0,06 & & 0,01 & 2,97 & 0,19 & 34,9 & 2,3 \\
\hline HAV2 P5-5 & & 48,4 & 4,8 & 140,5 & 3 & & 0,04 & 1,27 & 0,08 & 0,8 & 0,05 & & 0,01 & 2,73 & 0,16 & 51,5 & 3,2 \\
\hline HAV2-P5-8 & 4 & 38,5 & 3,9 & 176,4 & 3,2 & 0 , & 0,05 & 1,37 & 0,08 & 0 & 0,06 & & 0,01 & 2,86 & 0,18 & 61,7 & 4,0 \\
\hline HAV2 P6-1 & 530 & 50,1 & 5,0 & 209,3 & 4,7 & 0,44 & 0,04 & 1,38 & 0,09 & 0,9 & 0,06 & 0,1 & 0,01 & 2,83 & 0,18 & 73,9 & 5,0 \\
\hline HAV2 P6-2 & 550 & 49,5 & 5,0 & 262,7 & 6,9 & 0,44 & 0,04 & 1,42 & 0,09 & 0,91 & 0,06 & 0,10 & 0,01 & 2,87 & 0,18 & 91,5 & 6,2 \\
\hline
\end{tabular}

Tab. 2 : Résultats des mesures réalisées dans le cadre des datations OSL.

Tab. 2: Results of the measurements performed in the frame of OSL datings. 
et $100 \%$ de sa saturation (Aitken, 1985). La contribution du rayonnement alpha à la dose annuelle utilise un coefficient d'efficacité car les rayons alpha sont peu efficaces à produire un signal d'OSL. Ce facteur (non reporté dans le tableau) a été mesuré pour chaque échantillon et varie de 0,039 à 0,045 , valeurs proches de 0,04 habituellement utilisée (Rees-Jones \& Tite, 1997). Enfin, la contribution du rayonnement cosmique est calculée en utilisant la profondeur de l'échantillon depuis la surface (tab. 2B) et la densité (environ 2,0) du sédiment le surplombant (Prescott \& Stephan, 1982 ; Prescott \& Hutton, 1994). L'ensemble des débits annuels de dose et des paléodoses mesurées par SAR permettent de calculer les âges de dix-sept échantillons et leurs incertitudes analytiques qui sont reportés dans les deux dernières colonnes du tableau 2B.

\subsubsection{2 - Résultats}

Dix âges ont été obtenus pour les échantillons de la section Hav.1 et sont reportés dans la figure 4. Ils sont répartis entre 67,6 et 28,9 ka. L'ordre stratigraphique est respecté sauf pour le point (Hav.1 P1-7) qui se caractérise par un âge plus jeune difficile à interpréter. Rien dans les propriétés OSL ne le distinguant des autres échantillons, il faut donc envisager une exposition partielle à la lumière, soit pendant le prélèvement, soit pendant la préparation, ou bien le prélèvement involontaire de sédiments correspondant à un terrier comblé postérieurement (fréquent dans cette zone). Cet échantillon n'ayant été prélevé qu'une seule fois, il n'a pas été possible de confirmer l'une de ces hypothèses et il convient de ne pas tenir compte de cette valeur.

Les sept datations des échantillons de la section Hav.2 sont reportées dans les figures 6 et 7 et recouvrent 1'intervalle de 91,5 à 28,4 ka. On constate comme pour la section Hav.1 que l'ordre stratigraphique est respecté. De plus, la position stratigraphique du point Hav.2 P5-4 est la même que celle des os de mammifère datés par le radiocarbone. Leurs âges OSL et ${ }^{14} \mathrm{C}$ sont identiques $34,9 \pm 2,3 \mathrm{ka}(\mathrm{OSL})$ et $33,47 \pm 0,20 \mathrm{ka}$ cal. BP et $32,33 \pm 0,21 \mathrm{ka}$ cal. BP $\left({ }^{14} \mathrm{C}\right.$ calibré $)$ d'autre part. Ce bon accord renforce la confiance que l'on peut accorder aux modalités des calculs dosimétriques. Par ailleurs, comme on pouvait s'y attendre, les squelettes de marmotte et de spermophiles préservés dans les colmatages des terriers trouvés dans l'unité 7 sont plus jeunes ( 44-46 ka) que l'âge OSL (51,5 $\pm 3,2 \mathrm{ka})$ mesuré pour le sommet de cette unité (Hav.2 P5-5). Les résultats des datations OSL sont par ailleurs discutés plus en détail dans la synthèse de la partie 4 qui les intègre à l'ensemble des autres données.

\subsubsection{3 - Les autres datations $\left({ }^{14} \mathrm{C}\right.$ sur os, TL-silex, ESR-U/Th sur dent mammifère)}

Huit restes fauniques non brûlés, provenant de l'unité $6 \mathrm{a}$, ont été sélectionnés afin de réaliser des datations ${ }^{14} \mathrm{C}$ AMS et dater ainsi le niveau d'occupation paléolithique Hav.2-N2. Des résultats significatifs et probants n'ont été obtenus que pour deux d'entre eux qui présentaient suffisamment de collagène pour être datés (tab. 3).

Les résultats obtenus sont les suivants : $27020 \pm 140 \mathrm{BP}$ et $28100 \pm 180$ BP. Une fois calibrées (tab. 3), ces deux dates donnent un âge moyen d'environ $33 \mathrm{ka}$ pour le niveau archéologique Hav.2-N2 sur la base de la calibration de Fairbanks et al. (2005), et un peu plus jeune $\sim$ 31,5 ka, en utilisant IntCal13 (Reimer et al., 2013).

Par ailleurs, deux datations ${ }^{14} \mathrm{C}$ ont été réalisées sur des restes de rongeurs (un ensemble comprenant un tibia, un fémur et un coxal de marmotte et deux squelettes de spermophiles) préservés dans le colmatage des terriers qui affectent l'unité 7 (42020 \pm 380 BP et $40030 \pm 370$ BP). Après calibration, les âges obtenus (tab. 3) sont cohérents avec les datations OSL et permettent de resserrer la fourchette chronologique du sommet de l'unité 7. Elles confirment aussi l'existence d'une surface de sol stable autour de 44-46 ka au sommet de l'unité 7 antérieure à l'ouverture du réseau de fentes F-5 et l'importance du hiatus entre les unités 7 et 6 .

Deux datations TL-silex ont été effectuées sur des artefacts lithiques chauffés provenant de la base de l'unité 12 par N. Debenham et ont donné les résultats suivants : 106-130 ka et 92-112 ka. Les résultats sont nettement discordants et par ailleurs sensiblement plus anciens que les âges OSL obtenus dans cette unité. Cette observation semble confirmer l'importance des processus de remaniement de matériaux issus de sols plus anciens au sein de l'unité 13 suggérés par les données micromorphologiques et l'étude sédimentologique. Aucun résultat de datation de dent de grand mammifère par la méthode ESR-U/Th n'est disponible pour l'instant.

\begin{tabular}{|c|c|c|c|c|c|c|}
\hline Unité & Matériel & Labo & Age ${ }^{14} \mathrm{C} B P$ & $\begin{array}{l}\text { Age cal. moy. } \\
\text { [yr cal BP] } \\
\text { (Fb-2007)* }\end{array}$ & $\begin{array}{l}\text { Gamme d'âge calibré } \\
\text { [yr cal BP]** } \\
\text { (prob. } 2 \text { sigmas) }\end{array}$ & $\begin{array}{l}\text { Age } \\
\text { moyen** }\end{array}$ \\
\hline $6 \mathbf{6 a}$ & $\begin{array}{l}\text { Os de cheval (niveau } \\
\text { archéologique Hav.2- } \\
\text { N2) }\end{array}$ & $\begin{array}{l}\text { Beta - } \\
307416\end{array}$ & $27020 \pm 140$ & $32328 \pm 215$ & $30850-31260$ & 31063 \\
\hline 6a & $\begin{array}{l}\text { Os de bison (niveau } \\
\text { archéologique Hav.2- } \\
\mathrm{N} 2 \text { ) }\end{array}$ & $\begin{array}{l}\text { Beta - } \\
332604\end{array}$ & $28100 \pm 180$ & $33470 \pm 244$ & $31410-32560$ & 31893 \\
\hline $\begin{array}{l}7 \\
\text { (crotovines) }\end{array}$ & $\begin{array}{l}\text { Os : tibia, fémur et } \\
\text { coxal de marmotte }\end{array}$ & $\begin{array}{l}\text { Beta - } \\
328569\end{array}$ & $42020 \pm 380$ & $46495 \pm 391$ & $44660-46060$ & 45358 \\
\hline $\begin{array}{l}7 \\
\text { (crotovines) }\end{array}$ & $\begin{array}{l}\text { Os : squelette complet } \\
\text { de spermophile }\end{array}$ & $\begin{array}{l}\text { Beta - } \\
328570\end{array}$ & $40030 \pm 370$ & $44751 \pm 375$ & $43000-44380$ & 43653 \\
\hline
\end{tabular}

Tab. 3 : Datations ${ }^{14} \mathrm{C}$ des restes osseux (calibrations d'après Fairbanks et al. $(2005) *$ et Reimer et al. (2013), IntCal13**).

Tab. 3: ${ }^{14}$ C datings of bone remains (calibrations according to Fairbanks et al. (2005)* and Reimer et al. (2013), IntCal13**). 


\section{3 - DONNÉES BIOCLIMATIQUES}

\section{1 - MALACOLOGIE (O.M.)}

Les échantillons des deux séries ont été tamisés sous l'eau à $425 \mu \mathrm{m}$, puis séchés (cf. partie 2.1.6). Les coquilles entières et les fragments uniques ont été triés et identifiés, puis les individus dénombrés selon la méthode de comptage mise au point par Ložek (1964). Le nombre d'individus par assemblage, ou abondance, varie entre 0 et 413 sur Hav.1-P1 et entre 2 et 394 sur Hav.2-P5 (fig. 17). Le nombre d'espèces, ou richesse, varie entre 0 et 4 sur Hav.1-P1 et entre 2 et 6 sur Hav.2-P5.

Sur la base de synthèses taxonomiques (Anderson, 2005 ; Bank et al., 2001 ; Falkner et al., 2002 ; Rocroi et al., 2007) et écologiques (Adam, 1960; Germain, 1930, Kerney et al., 1983, Ložek, 1964, Zilch \& Jaeckel, 1962), nationales et européennes, ont été identifiés cinq taxons terrestres, i.e. Pupilla muscorum (Linnaeus, 1758), taxon euryèce de milieu découvert, $P$. alpicola (Charpentier, 1837), taxon palustre actuellement montagnard préférant les environnements calcaires, Trochulus hispidus (Linnaeus, 1758) (une présence unitaire sur Hav.1-P1), taxon mésophile excepté dans les stations trop sèches, Succinella oblonga (Draparnaud, 1801), taxon hygrophile de milieu ouvert à végétation clairsemée, les limaces et quatre taxons aquatiques, i.e. Gyraulus crista (Charpentier, 1837), Planorbis planorbis (Linnaeus, 1758), Valvata cristata (Müller, 1774) et V. piscinalis (Müller, 1774), chacun représenté par une présence unitaire sur Hav.2-P5. Ces quatre taxons aquatiques préfèrent les milieux d'eau stagnante, ou à écoulement lent, riches en plantes, voire encombrés de végétation.

La composition, l'abondance et la richesse de ces prélèvements sont dans la norme des malacofaunes des loess de la façade nord-ouest de l'Europe continentale qui sont le plus souvent dominées par tout ou partie des quelques taxons identifiés à Havrincourt, i.e. P. muscorum (milieu ouvert, euryèce), T. hispidus (mésophile), S. oblonga (hygrophile, milieu découvert) et $P$. alpicola (palustre, montagnard) (Moine, 2008, 2014). Les milieux associés sont le plus souvent pauvres en raison de conditions topographiques et climatiques défavorables au développement d'une végétation diversifiée.

\subsection{1 - Description des malacofaunes associées aux unités sédimentaires identifiées}

Dans ce travail de synthèse, seule une description et, plus avant, une analyse préliminaire des malacofaunes sont présentées afin de compléter l'interprétation des unités pédostratigraphiques.

- Unité 7 : en raison de sa nature décarbonatée, seuls quelques fragments de coquilles peuplent les échantillons, certains étant même stériles. Ces malacofaunes pauvres et biaisées à cause de la dissolution de la majeure partie du matériel sont peu exploitables.

- Unité 6a : sur Hav.1-P1, la malacofaune apparaît dans cette unité en raison de son caractère légèrement carbonaté $\left(\mathrm{CaCO}_{3}: 0,5\right.$ à $\left.3,5 \%\right)$, s'accroît très légèrement, mais reste très peu abondante et dominée par le taxon de milieu découvert sec à modérément humide Pupilla muscorum. Sur Hav.2-P5, la malacofaune augmente aussi, mais est nettement plus abondante, alors que le taux de carbonate y est similaire (2 à $5 \%$ ). Par contre, si P. muscorum domine le milieu à la base, elle ne fait ensuite que co-dominer en proportions à peu près équivalentes d'abord avec $P$. alpicola et Succinella oblonga, puis uniquement avec $S$. oblonga suite au décrochement de $P$. alpicola dans les trois échantillons

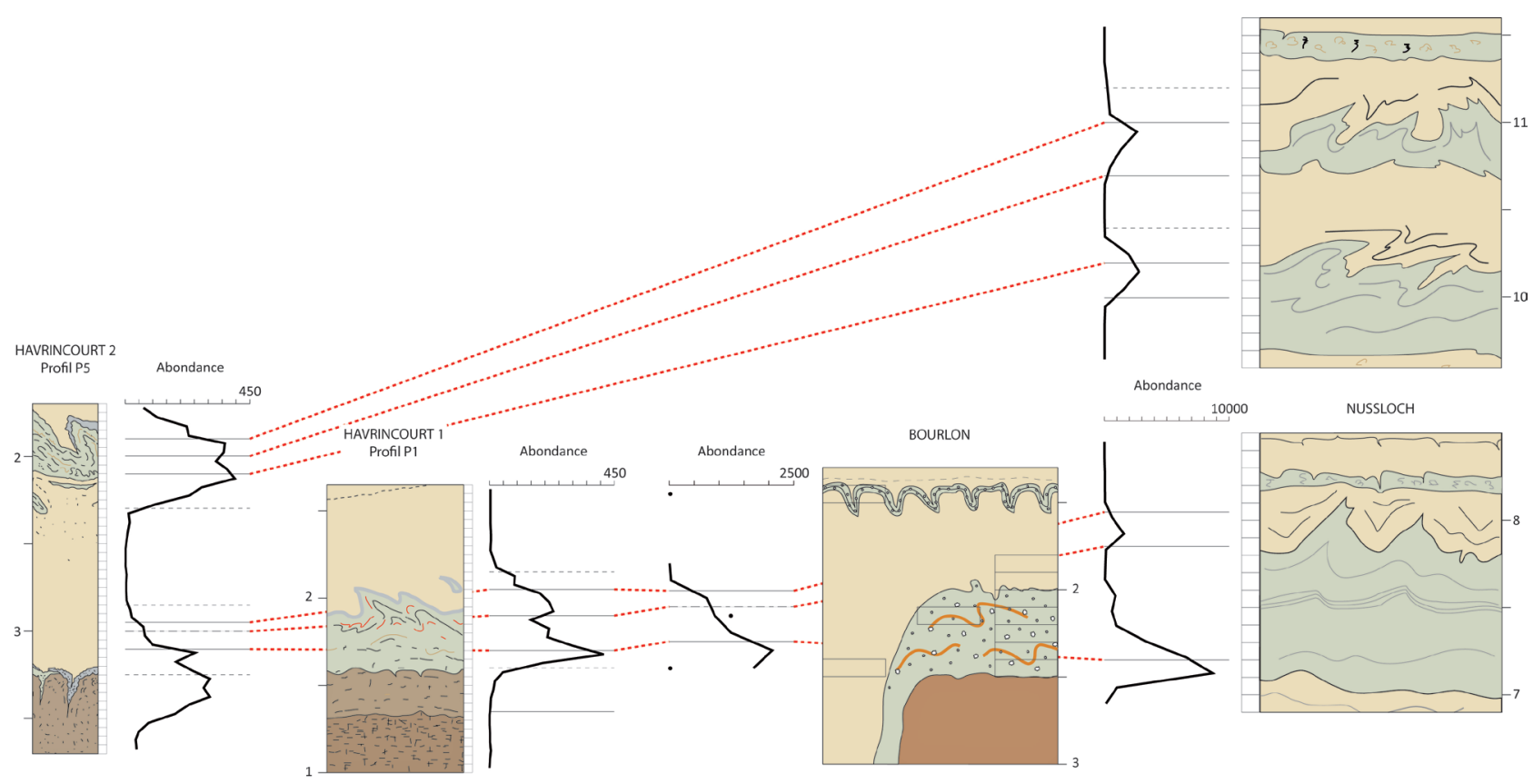

Fig. 17 : Variations de la dynamique de l'abondance totale en mollusques à travers les gleys de toundra d'Havrincourt, Bourlon et Nussloch (gley de toundra G2).

Fig. 17: Variations of the total mollusc abundance dynamics throughout the tundra gleys of Havrincourt, Bourlon and Nussloch (tundra gley G2). 
supérieurs affectés par des fentes en coin remplies de matériel gleyifié. On y note aussi la présence de deux individus aquatiques probablement en raison de l'existence de petits plans d'eau localisés résultant de la fonte des coins de glace.

- Unité 5 : sur Hav.1-P1, la courbe de l'abondance totale, ainsi que celles de chacun des quatre taxons principaux, montrent deux pics d'abondance : le premier à la base est élevé et le second au sommet est de moindre importance. P. muscorum domine dans le premier pic et co-domine avec le taxon hygrophile $S$. oblonga dans le second. En outre, dans les phases d'abondance maximale de chaque pic, ses proportions sont décroissantes au contraire de celles de $S$. oblonga et du taxon palustre $P$. alpicola. La proportion en limaces baisse de moitié dans le pic sommital. Sur Hav.2-P5, malgré un positionnement de la colonne d'échantillonnage là où l'unité 5 est la moins épaisse (fig. 6), les changements de la malacofaune reflètent plutôt ceux attendus au travers de ce gley de toundra là où son épaisseur est normale. En l'absence de vérification in situ lors du prélèvement, on peut donc supposer que c'était le cas suffisamment près en arrière du front de taille pour que les échantillons s'en trouvent affectés. En effet, les valeurs très faibles caractérisant les loess ne sont atteintes que dans l'échantillon 290-295 (échantillon de $5 \mathrm{~cm}$ prélevé entre 2,90 et 2,95 m). Dans l'intervalle, on note un maximum d'abondance au milieu de l'unité 5a, suivi d'un épaulement (ralentissement dans la baisse de l'abondance) à la transition entre les unités $5 \mathrm{a}$ et $5 \mathrm{~b}$. P. muscorum co-domine avec $S$. oblonga, et les proportions de $P$. alpicola montrent une tendance croissante, dans les deux pics. Dans l'épaulement, on note aussi la présence de deux individus aquatiques, et une proportion en limaces deux fois moindre que dans le pic basal.

- Unité 4 : sur Hav.1-P1, l'abondance totale, bien que décroissante, et la richesse sont encore assez élevées à la base de l'unité en raison de la présence d'injections de gley de toundra riches en coquilles. Ces deux paramètres décroissent ensuite nettement lorsque l'unité devient purement loessique. Seuls subsistent $S$. oblonga et des fragments non déterminables de Pupilla probablement attribuables à $P$. muscorum étant donné le contexte généralement plus sec associé aux phases de dépôt des loess. Sur Hav.2-P5, les abondances sont également très faibles et les mêmes remarques peuvent être faites à propos des taxons qui se maintiennent, incluant ici les limaces.

- Unité 3 : sur Hav.2-P5, seul enregistrement disponible, les taxons précédemment disparus réapparaissent et les abondances augmentent de nouveau fortement. L'allure de la courbe d'abondance totale est bimodale avec un premier pic dans l'échantillon 210-215 et un second dans l'échantillon 190-195. La position relative de ces deux pics dans l'unité est difficile à indiquer en raison de sa forte cryoturbation. La malacofaune est dominée par S. oblonga, P. muscorum est minoritaire et les proportions de ces deux taxons sont constantes. Chaque pic d'abondance est souligné par des proportions plus élevées du taxon palustre $P$. alpicola au détriment des limaces.

\section{2 - LA FAUNE MAMMALIENNE (P. AG.)}

Le gisement d'Havrincourt a livré 467 restes fauniques dont 284 proviennent des quatre niveaux d'occupation paléolithiques (tab. 4). Sept espèces ont été déterminées de façon certaine. Il s'agit de : Bison priscus, le bison des steppes ; Equus sp. de grande taille, le cheval ; Equus sp., un cheval de plus petite taille ; Rangifer tarandus, le renne ; Coelodonta antiquitatis, le rhinocéros laineux ; Mammuthus primigenius, le mammouth laineux ; Mustela eversmanni, le putois des steppes. A ces sept espèces viennent s'ajouter des restes de marmottes, d'arvicolidés et de spermophiles. Ces rongeurs, présents de manière intrusive au sein de la séquence, ont été principalement découverts en contexte de terrier. L'ensemble de ces restes a été mis au jour au sein des deux secteurs de fouille, Hav.1 et Hav. 2. Le secteur 1 est le plus pauvre en nombre de restes et en information d'ordre stratigraphique et palethnographique, il permet néanmoins de mettre en évidence un cortège faunique très cohérent avec les restes retrouvés au sein du secteur 2 .

\begin{tabular}{|l|r|l|r|r|r|}
\hline $\begin{array}{l}\text { Taxon / Unité } \\
\text { stratigraphique }\end{array}$ & Hav.1-N3 & Hav.2-N0 & Hav.2-N1 & Hav.2-N2 & $\begin{array}{l}\text { hors niveau } \\
\text { archéologique }\end{array}$ \\
\hline Bison & 4 & 1 & 0 & 7 & 9 \\
\hline Cheval & 13 & 2 & 3 & 21 & 17 \\
\hline "petit cheval" & 0 & 0 & 0 & 2 & 1 \\
\hline Renne & 0 & 0 & 0 & 12 & 4 \\
\hline Rhinocéros & 3 & 0 & 0 & 4 & 12 \\
\hline Mammouth & 0 & 0 & 0 & 0 & 4 \\
\hline Grand herbivore & 4 & 0 & 5 & 40 & 22 \\
\hline Petit herbivore & 0 & 0 & 0 & 16 & 0 \\
\hline Putois des steppe & 0 & 0 & 0 & 0 & 1 \\
\hline Arvicolidé & 2 & 0 & 0 & 0 & 19 \\
\hline Marmotte & 2 & 0 & 0 & 1 & 16 \\
\hline Spermophile & 36 & 0 & 3 & 0 & 35 \\
\hline Non déterminé & 6 & 0 & 3 & 135 & 141 \\
\hline Total & 70 & 3 & 15 & 238 & \\
\hline
\end{tabular}

Tab. 4 : Dénombrement des vestiges mammaliens par espèces et par niveaux archéologiques.

Tab. 4: Counting of large mammal remains by species and by archaeological levels. 


\subsection{1 - Analyse de la faune de Hav.1}

Le matériel découvert au sein du secteur 1 correspond à 30 restes de grande faune (Coelodonta antiquitatis, Bison priscus, Equus sp.) et 40 restes de micromammifères (Marmota sp., Citellus sp. et Arvicolidé).

Le rhinocéros laineux (Coelodonta antiquitatis) est représenté en particulier par deux os retrouvés proche l'un de l'autre, pouvant anatomiquement appartenir au même individu, un métatarsien III droit et un tibia droit (fig. 18A). Ces deux os montrent que la forme présente à Havrincourt est le rhinocéros laineux typique du Pléistocène supérieur, que ce soit pour le métatarsien III (fig. 18B) ou le tibia (fig. 18C). Le tibia de l'individu du secteur 1 apparait plus gracile que celui provenant du secteur 2 , il pourrait caractériser une femelle, mais tous les deux s'insèrent parfaitement au sein des variations notées.

Cette faune témoigne d'un contexte écologique caractéristique, en l'occurrence un paysage de steppe sous un climat continental froid et sec. Le renne n'est pas présent, ce qui s'observe également pour le niveau paléolithique Hav.-N1 du secteur 2 et pourrait indiquer soit une occupation durant une phase peu froide, soit durant une saison où celui-ci n'est pas présent sur le territoire. Seuls seize restes fauniques sont clairement associés au niveau archéologique Hav.1-N3 contenu dans l'unité 12 (cf. partie 5.2).

D'un point de vue palethnographique, il est à noter qu'aucun des vestiges fauniques du secteur 1 ne porte de stigmates permettant de mettre en évidence une action anthropique. Il est donc délicat d'interpréter en terme comportemental une telle accumulation.

\subsection{2 - Analyse de la faune de Hav.2}

La fouille du secteur 2 a livré 397 restes dentaires et osseux de mammifères dont 268 sont clairement associés aux niveaux d'occupation humaine. La diversité taxinomique est relativement élevée avec au minimum dix espèces identifiées. La grande majorité des restes proviennent du niveau archéologique Hav.2-N2 représentant 238 restes au total (incluant plusieurs loci identifiés lors des fouilles). Ces 238 éléments sont a priori contemporains voire directement tributaires de l'occupation humaine. Le niveau archéologique Hav.2-N1 corrélé à la fin du Paléolithique moyen a livré quatorze restes. Six restes ont été récoltés dans le niveau archéologique N0. Toutes les autres unités stratigraphiques, sans indice de présence humaine, ont livré plus ou moins de matériel sans qu'aucune distinction stratigraphique ne soit réellement possible.

Les 43 éléments d'Equus sp. récoltés en font l'espèce la mieux représentée au sein du secteur 2 . Trois restes osseux témoignant d'un cheval de plus petite taille que la forme commune, sans pour autant atteindre les dimensions de l'hydrontin Equus hydruntinus, ont également été mis au jour. Ces deux formes sont essentiellement présentes dans le niveau Hav.2-N2. Le bison vient ensuite avec dix-sept restes (principalement un reste pour Hav.2-N0 et sept restes pour Hav.2-N2), suivi par le renne avec seize restes. Le renne est presque uniquement présent dans le niveau Hav.2-N2. Le rhinocéros laineux est présent avec seize restes également. Le mammouth laineux n'a été identifié qu'en contexte « naturel » au sein d'unités stratigraphiques sans artefact lithique, il n'est représenté que par quatre pièces.

Des ossements de grands herbivores ont été découverts, ils appartiennent soit au cheval soit au bison, ce qui tend à augmenter sensiblement la proportion de ces deux taxons. Le petit herbivore non attribué est à rapporter très probablement au renne, augmentant là aussi le nombre potentiel de pièces pour cet animal. Le dénombrement par nombre minimum d'individus s'est avéré peu instructif, de par la faible richesse en éléments pour chaque taxon par niveau. Par convention et de manière logique, en considérant que chaque unité stratigraphique est indépendante l'une de l'autre, un seul individu est présent par taxon et par unité, à quelques exceptions près : au moins un adulte et un jeune bison ont été dénombrés dans l'unité stratigraphique 8. Le niveau Hav.2-N1 a livré un adulte et un animal assez âgé, pour le cheval et au moins un très jeune animal et un adulte ont été décomptés au sein de l'unité 10. Le niveau Hav.2-N2 a livré au moins un adulte et un jeune renne.

Par conséquent, même si le nombre total d'individus identifiés pour chaque taxon est élevé à Havrincourt, celui-ci est corrélé uniquement avec le grand nombre d'unités stratigraphiques ayant livré parfois un seul reste et donc un seul individu. Les différents niveaux d'occupation humaine ne livrent que peu d'individus témoignant d'occupations très courtes, compte-tenu également des phénomènes de dégradations taphonomiques importants expliquant la disparition d'une partie du matériel faunique.

Le bison des steppes est représenté par quelques éléments dentaires et osseux. La diagnose spécifique a pu être effectuée en particulier à partir d'une série dentaire supérieure (fig. 18D). Dans la plupart des cas, les ossements de bovidé identifiés n'ont pas été déterminés mais attribués au bison plutôt qu'à l'auroch, le contexte écologique et les cortèges fauniques connus pour le Pléistocène supérieur en France septentrionale plaidant pour cette attribution (Auguste, 2009, 2012).

Le cheval est l'espèce dominante à Havrincourt, pour le niveau paléolithique moyen (Hav.2-N0 et Hav.2N1) comme pour le niveau du Paléolithique supérieur (Hav.2-N2). L'animal présente au niveau dentaire les caractéristiques caballines et peut être rapporté à l'une des formes connues durant le Pléistocène supérieur. Il est toutefois apparu au cours de l'analyse du matériel que trois pièces se distinguaient par rapport aux autres du point de vue de leur massivité. Ces éléments (un talus et deux fragments de métacarpiens) sont en effet sensiblement plus petits, mais ils n'atteignent pas non plus la gracilité caractéristique du petit équidé non caballin E. hydruntinus. Il est délicat de pouvoir attribuer ces éléments à une espèce différente de celle présente par ailleurs, un phénomène de dimorphisme 
B

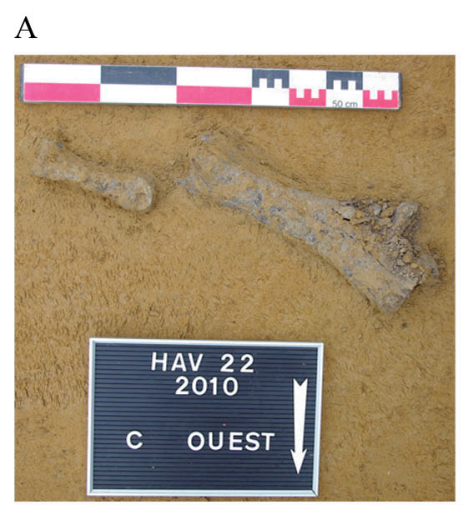

$\mathrm{D}$
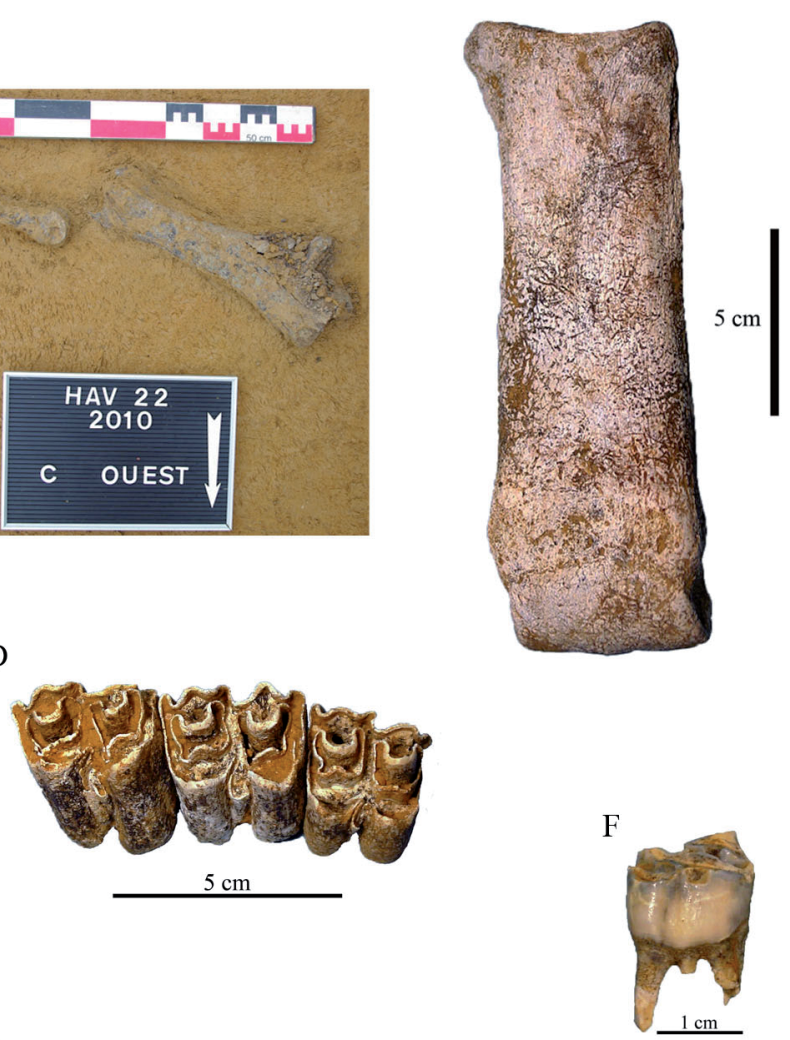

$\mathrm{E}$
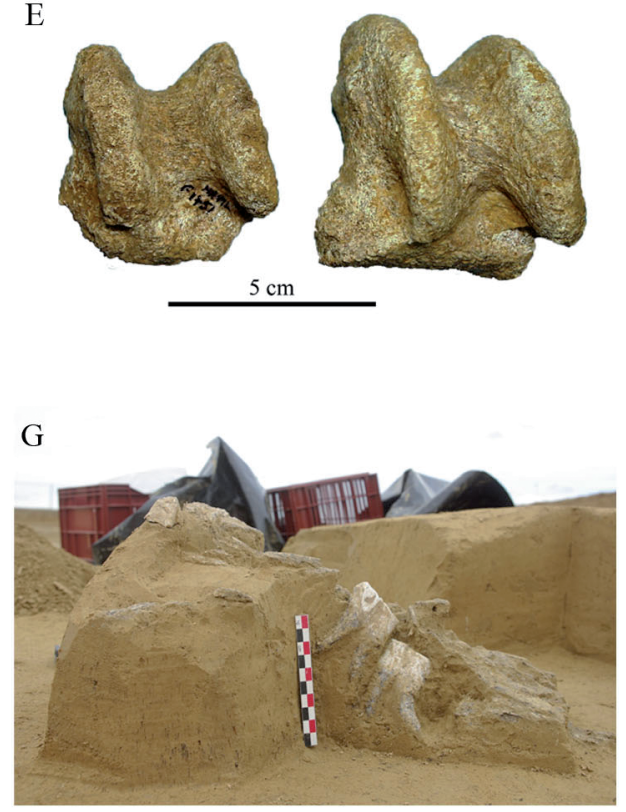

$\mathrm{C}$

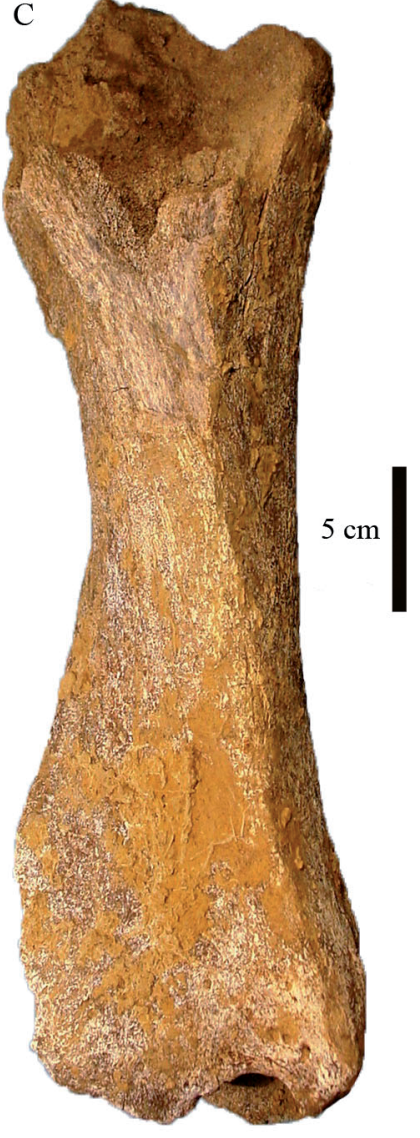

$\mathrm{H}$

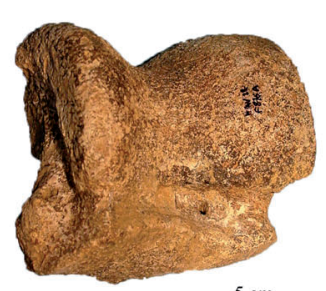

I

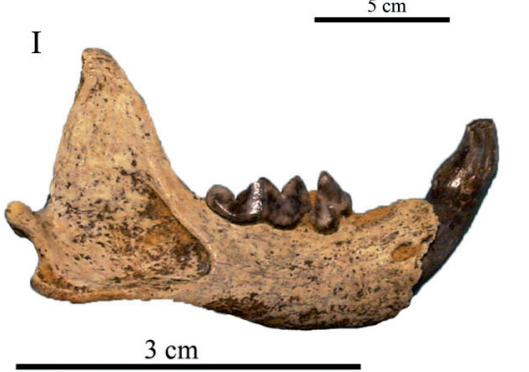

Fig. 18 : Hav.1 et Hav.2 - Macro- et méso-faune mammalienne.

A/ Hav.1, coupe ouest, métatarsien III et tibia droits de rhinocéros laineux, in situ. B/ Hav.1, coupe ouest, métatarsien III droit de rhinocéros laineux, vue dorsale. C/ Hav.1, coupe ouest, tibia droit de rhinocéros laineux, vue dorsale. D/ Hav.2, série dentaire supérieure droite (M1 à M3) de bison des steppes, vue occlusale. E/ Hav.2, talus gauches de cheval, « petite forme » à gauche, « grande forme » à droite, vues proximales. F/ Hav.2, quatrième prémolaire inférieure droite de renne, vue vestibulaire. G/ Hav.2, hémimandibule gauche (avec M1 et M2) de rhinocéros laineux, in situ, vue vestibulaire. H/ Hav.2, talus gauche de rhinocéros laineux, vue proximale. I/ Hav.2, hémimandibule droite (avec canine, P4 et M1) de putois des steppes, vue vestibulaire. A et $\mathrm{E}$ : clichés E. Goval ; B, C, D, F, G, H et I : clichés P. Auguste ; PAO : N. Sévêque.

Fig. 18: Hav.1 and Hav.2: large- and medium-size mammals. A/ Hav.1, west cut, right third metatarsian and right tibia of woolly rhinoceros, in situ. B/ Hav.1, west cut, right third metatarsian of woolly rhinoceros, dorsal view. C/ Hav.1, west cut, right tibia of woolly rhinoceros, dorsal view. D/ Hav.2, upper right teeth (M1-M3) of steppe bison, occlusal view. E/ Hav.2, left talus of horse, "little form" left, "big form" right, proximal views. F/ Hav.2, right lower fourth premolar of reindeer, vestibular view. G/ Hav.2, left hemimandible (with M1 and M2) of woolly rhinoceros, in situ, vestibular view. H/ Hav.2, left talus of woolly rhinoceros, proximal view. I/ Hav.2, right hemimandible (with canine, P4 and MI) of steppe polecat, vestibular view. A and E: photos E. Goval; B, C, D, F, G, H and I: photos P. Auguste; CAD: N. Sévêque. 
sexuel particulièrement bien marqué pouvant potentiellement être avancé. Nous avons préféré garder ce taxon sous la dénomination de « petite forme ». Les éléments dentaires du cheval d'Havrincourt, en particulier les dents supérieures, ont permis de les comparer avec ceux d'autres chevaux du Pléistocène supérieur. Ces dents apparaissent ainsi proches des E. germanicus de Combe Grenal et divergent par rapport aux E. gallicus anciens ou plus récents. C'est en particulier la faiblesse de l'indice protoconique qui intervient pour constater ce point. Celui-ci se confirme avec les dents inférieures qui semblent témoigner de la même tendance. Une portion mandibulaire portant deux déciduales provenant $\mathrm{du}$ niveau paléolithique moyen (Hav.2-N1) s'avère extrêmement intéressante. En effet, les deux dents lactéales sont à peine usées et indiquent donc que le jeune poulain a été abattu alors qu'il n'était âgé que de 30 à 45 jours d'après l'estimation fournie par Guadelli (1998). En prenant pour référence une naissance en mai, cette pièce témoigne donc de l'occupation humaine au début de l'été. Deux talus d'équidé ont également été identifiés, rapportés par leur taille l'un à la «petite forme » l'autre au cheval commun (fig. 18E). Les dimensions de ces deux pièces s'avèrent en fait assez proches et les deux os apparaissent plus graciles que pour d'autres chevaux. Il apparaît donc difficile de dénommer spécifiquement le cheval d'Havrincourt (ou les chevaux s'il s'agit de deux taxons bien distincts), mais celui-ci s'inscrit bien au sein de la variabilité observée pour les équidés du Pléistocène supérieur.

Le renne n'est présent qu'au sein du niveau Hav.2-N2 (Paléolithique supérieur ancien) et dans quelques unités stratigraphiques sans présence humaine, il est absent durant l'occupation du Paléolithique moyen (Hav.2-N1). Outre les éléments osseux, seul un reste dentaire a été retrouvé, une prémolaire inférieure mettant en évidence les caractères diagnostiques de l'espèce (fig. 18F). Les dimensions de cette dent montrent une longueur comparable à celle d'autres rennes pléistocènes, tandis que la dent est plus large. L'absence du renne au sein du niveau corrélé au Paléolithique moyen (Hav.2-N1) est intéressante, témoignant d'un climat plus clément que pour le niveau corrélé au Paléolithique supérieur ancien (Hav.2-N2), tout en demeurant continental avec un paysage dominé par la steppe. Une différence de saisonnalité d'occupation n'est pas à exclure, mais le manque de données pour les occupations relatives au Paléolithique supérieur ancien empêche d'aller au-delà de ces considérations.

Le rhinocéros laineux est présent au sein du niveau Hav.2-N2 et quelques autres unités mais comme pour le renne, il est absent du niveau corrélé au Paléolithique moyen (Hav.2-N1). Il a pu être identifié indubitablement en raison de la morphologie caractéristique de plusieurs ossements, en particulier une hémimandibule gauche portant deux molaires (fig. 18G). Dans le secteur 2, un tibia sub-entier, un pyramidal, un métacarpien III et un talus ont permis son identification (fig. 18H). Les dimensions de ces pièces mettent en évidence une proximité morphométrique avec les moyennes des rhinocéros laineux européens.

La présence du mammouth laineux est rare à Havrincourt et provient d'unités stratigraphiques sans indices de présence humaine (limon brun, unités 7,8 et 10 ). Un fragment de défense a été découvert ainsi qu'une portion de molaire.

Enfin, un des éléments intéressants découverts au sein des séries fauniques d'Havrincourt est une portion crânienne de putois des steppes, comprenant des fragments crâniens, des dents supérieures, et les deux hémimandibules avec une bonne partie des séries dentaires. La mandibule a permis de confirmer l'attribution au putois des steppes plutôt qu'à un autre mustélidé, avec sa massivité notable en particulier au niveau du corps mandibulaire et la convexité du bord basal de la branche horizontale observable sous la partie antérieure de la première molaire inférieure (fig. 18I), élément diagnostique de l'espèce (Mallye \& Guérin, 2002). Le putois n'est identifié que dans l'unité stratigraphique 10 en contexte de terrier.

De petits animaux fouisseurs comme Marmota sp., la marmotte ; Citellus sp., le spermophile ou l'arvicolidé sont présents de manière intrusive au sein de différentes unités stratigraphiques de la séquence. Ils ont majoritairement été découverts en contexte de terriers, sous forme d'amas osseux et dentaires. Ils ont été décomptés par groupe d'ossements.

Leur intérêt est de montrer que durant leur présence, les conditions écologiques étaient typiquement steppiques et non de type toundra, ces animaux comme le putois étant des fouisseurs. Tous les spécimens retrouvés semblent être liés à un ou plusieurs épisodes de mortalités lors des phases d'hibernation au sein des terriers (fig. 4, 6 et 7).

\subsection{3 - Paléoécologie}

La fouille des deux secteurs du gisement d'Havrincourt a permis de récolter près de 500 vestiges mammaliens. Associées dans plusieurs niveaux d'occupation paléolithique à des industries lithiques, ces faunes offrent l'opportunité de reconstituer les cadres chronologiques et écologiques des occupations humaines.

D'un point de vue taphonomique, l'état de conservation du matériel dénote des phénomènes d'altération, parfois notables, liés au contexte de dépôt et d'actions physico-chimiques post-dépositionnelles synsédimentaires. L'état de surface est de manière générale moyen à dégradé, une perte de matière par dissolution et desquamation, fissuration longitudinale, un concrétionnement calcaire est parfois attesté. Il est donc évident qu'une partie probablement importante du matériel faunique initialement présent au moment du dépôt a dû disparaître sous l'effet de la dissolution.

Certains restes fauniques sont retrouvés au sein de la séquence sédimentaire dans des unités stratigraphiques sans indice de présence humaine. Ces vestiges isolés correspondent très probablement à des animaux 
morts naturellement et recouverts par des dépôts loessiques. Pour les vestiges provenant des unités ayant livré également des artefacts lithiques, il semble cohérent de penser à une action anthropique en tant qu'agent accumulateur. La présence de stigmates liés à des actions de boucherie plaide pour cette interprétation comme 1'atteste l'analyse tracéologique (Goval et al., 2013).

Les cortèges fauniques mis en évidence dans les deux secteurs d'Havrincourt sont très cohérents. La faune est caractéristique du spectre d'espèces présentes couramment en France septentrionale durant les phases froides du Pléistocène supérieur au sein de la steppe à mammouth. Ainsi bison des steppes, cheval, renne, rhinocéros laineux, mammouth laineux, putois des steppes, marmotte et spermophiles sont bien les représentants types de cet écosystème présent durant les périodes froides (Auguste, 2009). Le climat est très continental, sec, avec des alternances saisonnières très marquées. Le paysage est dominé par les espaces fortement découverts de type steppique, avec une importante biomasse végétale uniquement disponible durant la belle saison. Aucun élément de faune n'indique la présence de zones boisées. C'est donc bien dans ce contexte que les deux occupations humaines les plus récentes vont se dérouler, à la fin du Paléolithique moyen et durant le début du Paléolithique supérieur. Une indication probable d'une phase plus froide peut être mise en évidence avec la présence du renne, absent au Paléolithique moyen mais bien identifié dans le niveau Hav.2-N2. Ce point est cohérent avec les modalités de dépôt sédimentaire. Cette globale homogénéité des associations fauniques limite l'interprétation biochronologique. Les animaux sont typiquement ceux rencontrés au cours du Pléistocène supérieur. Seul le cheval apporte des éléments intéressants, avec une affinité pour les formes proches de l'E. germanicus de la première partie du Pléistocène supérieur pour les niveaux les plus anciens, et proche de l'E. gallicus de Jaurens pour les plus récents (Valli \& Guérin, 2000). Il ne semble pas, par contre, correspondre avec les formes de la fin du Pléistocène supérieur, ce qui est cohérent avec le contexte chronostratigraphique et culturel. Cette affinité potentielle du cheval «paléolithique supérieur » d'Havrincourt avec le cheval de Jaurens s'observe également à Renancourt près d'Amiens où il est là encore associé à une industrie du début du Paléolithique supérieur (données fauniques inédites, P. Auguste). Concrètement, ce cheval apparaît plus gros que les chevaux de la fin du Pléistocène supérieur, dénommés Equus gallicus et $E$. arcellini, et pourrait s'apparenter soit à une forme évolué d'E. germanicus, soit une forme primitive d'E. gallicus comme cela s'observe à Jaurens. La faune de ce dernier gisement est datée aux environs de 28000 à 33000 BP (Mourer-Chauviré, 1980), ce qui serait donc en parfait accord avec les datations obtenues pour le secteur 1 de Renancourt 2 (Paris et al., 2013) et celle du niveau Hav.2-N2 d'Havrincourt (cf. partie 2.4.1.3).

\section{4 - SYNTHÈSE PÉDOSTRATIGRAPHIQUE; INTERPRÉTATION CHRONO-CLIMATIQUE, CHRONOSTRATIGRAPHIQUE ET CORRÉLATIONS (P.A., O.M., S.C.)}

Le contexte de versant en pente douce orienté au nordnord-est, dans lequel s'inscrit le gisement d'Havrincourt, a favorisé l'accumulation d'une couverture loessique de géométrie relativement régulière. Au sein de cette dernière, il a été possible d'individualiser plusieurs horizons périglaciaires particulièrement bien préservés et continus, aisément corrélables entre les deux secteurs éloignés de plus de $500 \mathrm{~m}$. La richesse exceptionnelle de l'information concernant les structures périglaciaires et les occurrences de pergélisol font d'Havrincourt une nouvelle séquence de référence pour le périglaciaire de la France septentrionale.

Ces niveaux périglaciaires constituent des horizons repères particulièrement importants pour la corrélation des séquences au niveau régional (Antoine et al., 2003a) et un préalable pour l'analyse des processus post-dépositionnels qui ont potentiellement affecté les niveaux archéologiques. Enfin, la méthode de décapage archéologique par paliers progressifs à la pelle mécanique a permis de mettre au jour à une profondeur de 3,5 m, et sur plus de $4000 \mathrm{~m}^{2}$, un réseau de grandes fentes en «V » à maille décamétrique, ce qui représente une première pour la France septentrionale (fig. 11-A et B).

Dans un premier temps, l'interprétation de la séquence d'Havrincourt se base sur la synthèse des données pédostratigraphiques et sédimentologiques et la corrélation des différents cycles dépôt-pédogenèse qui la compose avec le modèle pédosédimentaire régional de la France du nord-ouest. Cette analyse séquentielle, une fois couplée avec les données bioclimatiques (faunes) et des datations $\left(\mathrm{OSL}\right.$ et ${ }^{14} \mathrm{C}$ ), permet ensuite de proposer une interprétation chrono-climatique et chronostratigraphique ainsi que des corrélations à différentes échelles.

Sur la base de sa position stratigraphique, de ses caractéristiques pédologiques (horizon Bt interglaciaire), et les datations, l'horizon de sol brun lessivé de l'unité 15 est attribué au dernier interglaciaire (Eemien) et la série loessique qui le recouvre au Dernier Glaciaire (Weichselien). Deux grandes séquences de type glaciaire-interglaciaire sont donc individualisées : la séquence I (Fin Saalien / Interglaciaire-Eemien : unités 17 à 15 ) et la séquence II (Dernier Glaciaire (Weichselien) / Interglaciaire-Holocène : unités 14 à 0 ).

\section{1 - SÉQUENCE I : FIN SAALIEN (SIM 6) / INTER- GLACIAIRE EEMIEN (SIM 5E) ( 140-125 KA)}

La formation limoneuse basale d'Havrincourt $(\sim 1 \mathrm{~m})$ repose sur le substratum crayeux par l'intermédiaire d'un contact érosif majeur et irrégulier. Au-dessus de ce contact, la sédimentation débute avec la mise en place de limons loessiques compacts décarbonatés montrant dans leur majeure partie une structuration de type «limon à doublets » (unité 15a-c, fig. 7). Il s'agit de loess décarbonatés et plus ou moins fortement affectés par la 
pédogenèse liée au développement du sol interglaciaire sus-jacent (unité 15). Les valeurs de l'indice IGR (ratio : limons grossiers / limons fins + argile : 1,8) soulignent le très bon classement du sédiment et l'origine éolienne des particules.

Enfin, seul le profil Hav.2-P6 a montré des faciès loessiques calcaires homogènes (unité 16), épargnés par la décarbonatation liée au sol de l'unité 15 à la faveur d'une structure en creux localisée au toit de la craie. L'attribution de ces loess à la phase finale du Saalien (SIM 6) est basée sur l'absence de dépôts et sols quaternaires antérieurs, leur faciès de loess calcaire typique ainsi que sur la mise en évidence de structures périglaciaires indiquant des conditions particulièrement rigoureuses de type pléniglaciaire au cours de leur mise en place. Par ailleurs, on observe une quasi-absence de dépôts loessiques plus anciens en contexte de plateau; seules les séquences de couverture des terrasses de la Somme permettant de les décrire (Antoine, 1990, Antoine et al., 1994). Le niveau archéologique Hav.2-N0 se situe au sein de cette unité.

Le contact entre les faciès crayeux remaniés directement sus-jacents au substratum (unité 17) et les premiers loess montre des structures de cryoturbation intense avec injection de la craie (diapirs) dans la couche limoneuse sus-jacente. L'observation du décapage du palier le plus profond du secteur $2(-6,5 \mathrm{~m})$ a permis de mettre en évidence une organisation de type sol strié au sommet de la craie altérée (dégradation d'un réseau polygonal sur versant). Ces processus démontrent l'occurrence d'au moins une phase majeure de formation et de dégradation d'un pergélisol au sommet de la craie au cours de la fin du Saalien.

Les dépôts loessiques initialement carbonatés de la fin du Saalien sont par la suite systématiquement affectés par le développement d'un sol brun lessivé (horizon Bt de Luvisol), dont il ne subsiste le plus souvent que la racine profonde $(0,50$ à $1 \mathrm{~m})$. L'altération pédogenétique se matérialise par quelques biopores et par sa structure polyédrique. En lame mince s'observent des ferri-argilanes brun-orangé limpides. Les caractères pédogenétiques sont soulignés par une augmentation des pourcentages d'argile (24\%), et de carbone organique total (COT) autour de $0,3 \%$ (0,1 \% dans les loess), ainsi que par une augmentation globale des valeurs de susceptibilité magnétique. Le développement de ce sol à partir des loess calcaires saaliens (unité 16) se traduit par la formation d'un épais horizon à doublets argileux (unité 15b-d) limité à sa base par le front de décarbonatation. Compte tenu de sa position dans la stratigraphie et de son faciès, ce sol est attribuable au dernier interglaciaire (Eemien) et corrélable avec les sols de Rocourt ou d'Elbeuf 1 (Lautridou, 1987). Enfin, l'approche micromorphologique et l'observation détaillée de ce faciès dans la paroi nord de la fouille du secteur 2 (fig. 2, P7) a montré des faciès de type doublets (fig. 11-G) surimposés sur une forte structuration sédimentaire du matériel (laminations discordantes, structures lenticulaires) qui pourrait indiquer une phase de remaniement des loess par ruissellement avant le début des processus de pédogenèse interglaciaire. Ce processus a déjà été observé à Villiers-
Adam (Val d'Oise) où le Bt du sol interglaciaire s'est formé sur une topographie vallonnée formée entre la fin du dépôt des loess saaliens et le début de l'interglaciaire (Antoine et al., 2003b). A titre d'hypothèse il est envisagé que cette dynamique résulte de la disparition rapide, par fusion, du pergélisol à la fin du Saalien (allongement de la période de dégel estivale).

\section{2 - SÉQUENCE II (WEICHSELIEN-HOLOCÈNE) : 112-0 KA}

Compte tenu de la richesse du bilan de la séquence II, cette dernière a été subdivisée en cinq sous-séquences d'épaisseurs et de durées variables. La synthèse des données (terrain, analyses et datations) complétée par une comparaison avec la structure des enregistrements pédo-sédimentaires de référence de la France septentrionale, permet de définir les grands ensembles chronoclimatiques suivants :

- sous-séquence II-1 : Début Glaciaire (DGL) : unités $14 \mathrm{a}-14 \mathrm{~b}-14 \mathrm{c}$ et $15 \mathrm{R}$

- sous-séquence II-2 : Pléniglaciaire inférieur (PGI) : unités 13 à 8

- sous-séquence II.3 : Pléniglaciaire moyen (PGM) : unités 7 et 6

- sous-séquence II.4 : Pléniglaciaire supérieur (PGS) : unités 5 à 2

- sous-séquence II.5 : Tardiglaciaire-Holocène : unités 1 à 0

\subsection{1 - Sous-séquence II-1: Complexe de sols humi- fères / Début Glaciaire (DGL 112-70 ka)}

Dans tous les profils d'Havrincourt, la sousséquence II-1 débute, après un hiatus érosif au sommet du Bt de l'unité 15, par la mise en place d'un complexe de sols humifères dont la préservation est extrêmement irrégulière en raison de processus de dissolution du substratum crayeux (puits) contemporains de sa formation et d'érosions ultérieures. Dans le secteur 1, quand ils sont préservés, les horizons directement sus-jacents au sol brun lessivé de l'unité 15 sont représentés par une faible épaisseur $(0,5 \mathrm{~m})$ de limons brun-gris humifères non calcaires (unité $14 \mathrm{a}$ et b). L'interface des unités 14 et 15 est soulignée par un colmatage de silts lavés qui pénètrent l'unité 15a par l'intermédiaire d'un réseau de fines fentes irrégulières (glosses) de 15 à $30 \mathrm{~cm}$ de profondeur. La partie supérieure (unité 14a), séparée de l'unité $14 \mathrm{~b}$ par une limite diffuse, présente une structure plus homogène, granulaire et de nombreuses concrétions ferro-manganiques. D'un point de vue analytique, ces horizons se caractérisent par des taux de carbone organique total particulièrement élevés $(0,5 \%)$ typiques des horizons de sol humifère du Début Glaciaire weichselien. Au sein du profil Hav.2-P4, ils sont limités à leur sommet par un contact érosif matérialisé par un petit cailloutis de silex diffus.

Par ailleurs, dans les profils Hav.2-P4 et P9, de grands puits de dissolution karstique à remplissage polygénique 
ont été observés (fig. 8, 9 et 11-D). L'origine karstique de ces structures a pu être démontrée dans le profil P9 qui a permis d'observer à environ trois mètres de la surface de la poche un affaissement du toit de la craie et la présence d'une couche argilo-silteuse à concentration de manganèse au contact du remplissage (horizon de blocage des argiles de décarbonatation $\mathrm{B}_{\beta}$, unité $17 \mathrm{a}$ ). Ces puits, dont la formation a débuté dès la fin de l'Interglaciaire, sont tout d'abord colmatés par un assemblage hétérogène composé de nodules et blocs décimétriques de limons argileux brun-rouge (unités 14 et 15).

Les vides inter-nodules sont colmatés par des silts lités gris clair à blancs lavés matérialisant un drainage vertical du sol très intense (fig. 11-F). Ces processus représentent la signature de l'impact du gel-dégel et des processus de drainage brutaux des sols lors de la fonte de la couverture nivale dans un contexte climatique de type continental à forts contrastes saisonniers au cours du Début Glaciaire weichselien (Van Vliet-Lanoë, 1990 ; Antoine et al., 1994). L'association d'horizons de sols humifères acides (forêts boréales dominées par le pin) et de processus de fonte nivale très marqués pourrait expliquer l'intensification des processus de dissolution du substratum crayeux qui caractérise cette période. Il faut noter que plusieurs autres séquences régionales contemporaines du Début Glaciaire weichselien ont montré des structures de dissolution du même type : Marcoing (Sommé et al., 1980), Mautort (Antoine, 1990), Combles (Antoine, 1991), Bettencourt-Saint-Ouen (Antoine et al., 2003a) et Fresnoy-au-Val (Goval \& Locht, 2009).

La suite du colmatage intègre de moins en moins de matériaux remaniés à partir du Bt de l'unité 15 et passe progressivement à un ensemble beaucoup plus homogène brun noir montrant un faciès de sol gris forestier en place (unité 14b, fig. 11-G et K). En effet, l'étude micromorphologique montre des ferri-argilanes acajou à noirâtres (revêtements argilo-humiques) microlités abondants, en place dans la porosité de chenaux, même s'ils sont souvent légèrement fracturés. Ce sol, qui possède les caractéristiques typiques du sol gris forestier SS-1 du stratotype de Saint-Sauflieu, est attribué au deuxième interstade majeur du Début Glaciaire weichselien, contemporain du SIM 5 a entre $\sim 85$ et 80 ka et du GIS 21 .

La partie sommitale (unité 14a) montre enfin un faciès à laminations grossières qui témoigne d'une érosion de matériaux humifères remaniés (très grande abondance de papules et de fragments de ferri-argilanes acajou à brun foncé, accompagnés de papules de ferri-argilanes orangées de type $\mathrm{Bt}$ ) alimentés par l'érosion du sol gris forestier initialement présent sur les bords de la poche mais qui a ensuite totalement disparu. Dans les autres profils, le faciès de l'unité 14a apparaît cependant beaucoup plus homogène et correspond à un faciès de sol steppique sans illuviation d'argile que l'on peut rapporter au sol SS-2 de Saint-Sauflieu. Aucune datation OSL n'est disponible pour cet horizon et son interprétation repose donc uniquement sur les comparaisons avec la séquence régionale qui le place vers $70 \mathrm{ka}$ au niveau du GIS 20 (Antoine et al., 2003a). A ce niveau, le système de dissolution a définiti- vement cessé de fonctionner comme le montre l'absence totale de déformation au sein de l'ensemble des unités sus-jacentes.

\subsection{2 - Sous-séquence II-2 (unités 13 à 8 ) : Plénigla- ciaire inférieur (PGI 70-60 ka)}

Dans le secteur 1, la phase d'érosion postérieure au développement des sols humifères se manifeste sur une grande partie de l'emprise par la formation de structures chenalisées irrégulières, à remplissage lité limonosableux hétérogène, séparées par de gros blocs de sol humifère aux limites abruptes (anciens blocs gelés remaniés, nodules de sols humifères, silts blancs de ressuyage, inversion stratigraphique de certaines unités redéposées). Cette configuration indique une phase d'érosion intense et généralisée essentiellement liée à des processus de ruissellement et qui traduit une péjoration climatique importante directement postérieure au complexe de sols humifères (Pléniglaciaire inférieur). Aucune trace de grande fente à coin de glace n'a cependant été indiscutablement observée à ce niveau. La grande extension de ces structures permet d'envisager une violente déstabilisation du versant qui, par comparaison avec les données régionales et les dates OSL des unités directement susjacentes, semble s'être déclenchée à la transition Début Glaciaire / Pléniglaciaire inférieur.

Cet épisode sédimentaire au faciès hétérogène et chaotique passe vers le sommet à des faciès colluviaux lités (unité 13) puis à des faciès limoneux bruns plus homogènes intégrant du matériel d'origine éolienne (unité 12) qui signent un retour vers des conditions progressivement plus arides vers 65 ka d'après les dates OSL.

Les limons sableux de l'unité 13 (61-160 $\mu \mathrm{m}: 13 \%)$ remanient des lentilles des unités 14 et 15 et forment des corps sédimentaires pouvant dépasser plus d'un mètre d'épaisseur. Ces derniers faciès n'ont pas été observés sur le secteur 2 où le bilan sédimentaire de cette période est quasi nul (hiatus majeur). La présence dans ces dépôts d'artefacts laminaires remaniés à partir du complexe de sols humifères confirme l'importance de l'érosion qui leur est associée.

La suite de cette sous-séquence est représentée par la mise en place d'un limon brun non calcaire (unité 12). Cet horizon à texture très homogène s'individualise par un stock en limons grossiers plus important (20-61 $\mu \mathrm{m}$ : $56 \%$ ) et une diminution drastique des valeurs de susceptibilité magnétique. Cette unité pourrait être interprétée comme les premiers dépôts loessiques du Pléniglaciaire inférieur qui ont recouvert le versant de manière assez homogène $(0,3-0,50 \mathrm{~m})$. Les âges OSL de $67,6 \pm 3.9 \mathrm{ka}$ et de $65,0 \pm 3.8 \mathrm{ka}$, obtenus respectivement au milieu de l'unité 12 et juste au dessus dans l'unité 10, semblent compatibles avec une attribution au Pléniglaciaire inférieur (fig. 4). Cet horizon brun peu évolué au sein duquel se localise le niveau archéologique Hav.2-N1 (Paléolithique moyen récent) se matérialise sur le terrain par un faciès légèrement plus foncé dans les $\sim 20 \mathrm{~cm}$ supérieurs (COT : 0,34-0,38 \%), associé à une bioturbation et une 
agrégation plus importantes. Il montre des traces d'activité biologique (pédotubules) et une légère augmentation du taux d'argile (15 à $22 \%$ ) qui semblent traduire une légère pédogenèse (petit sol de type brun boréal ou de prairie arctique). Néanmoins, les valeurs de susceptibilité magnétique ainsi que les argiles et le COT se caractérisent par une décroissance de la base au sommet de cette unité qui plaide plutôt pour une origine essentiellement colluviale à partir de l'érosion des niveaux sous-jacents plus riches en argile et en matière organique. Enfin, la courbe des carbonates traduit une légère augmentation dans les $10 \mathrm{~cm}$ supérieurs $(\sim 1 \%)$ qui signale le début d'un nouvel épisode d'apports loessiques carbonatés. D'une manière générale, il n'est donc pas évident que cet horizon que l'on peut dénommer « limon brun d'Havrincourt » traduise une amélioration climatique notable. Il s'agit vraisemblablement d'un faciès essentiellement colluvial terminant la grande phase d'érosion qui se déclenche au sommet du complexe de sols humifères au début du Pléniglaciaire inférieur. Le caractère colluvial est d'ailleurs cohérent avec la forte dispersion des âges OSL entre les secteurs 1 et 2 ainsi qu'au sein de la même unité dans le profil Hav.2-P6 (73,9 \pm 5.0 et 91,5 $\pm 6.2 \mathrm{ka})$. Ces distorsions dans les âges résultent vraisemblablement de la présence de nombreux agrégats nodules de sols plus anciens contenant des grains de quartz non exposés à la lumière pendant le dépôt.

On remarque que l'âge le plus ancien $(91,5 \pm 6.2 \mathrm{ka})$, totalement en désaccord avec la stratigraphie, correspond par contre à une période compatible avec les éléments d'industrie laminaire habituellement datés du SIM 5c-5d dans le nord de la France (Révillion \& Tuffreau, 1998 ; Locht et al., 2002 ; Goval \& Hérisson, 2006 ; Goval, 2012). Ceci confirme clairement le caractère remanié de cette industrie et l'importance des processus de remaniement sédimentaire dans l'unité 13 .

$\mathrm{Au}$ niveau régional, ce faciès possède un équivalent probable dans la séquence voisine d'Hermies fouillée par $\mathrm{L}$. Vallin et $\mathrm{B}$. Masson à quelques kilomètres au sud-sudouest le long du Canal du Nord, où il se place à la fin de la séquence de colluvions litées à nodules de sols remaniés « limons lités d'Hermies », et potentiellement dans le profil de Combles dans la même position stratigraphique (Antoine, inédit).

Le sommet de l'unité 12 est ensuite affecté par une dégradation climatique marquée par le développement d'un horizon hydromorphe très peu épais, « micro-gley » (unité 11) lié à l'augmentation de l'humidité de surface (gel-dégel). S'ensuit la mise en place de matériel plus nettement loessique (unité 10) mais toujours pauvre en $\mathrm{CaCO}_{3}$ (décalcification liée à la faible épaisseur du dépôt et au gley sus-jacent ?) et daté de $65,0 \pm 3.8 \mathrm{ka}$ dans le profil Hav.1-P1. L'unité 9 qui recouvre systématiquement ce loess correspond à un gley de toundra typique de teinte grisâtre à taches d'oxydation et est aussi marquée par la diminution des apports loessiques allochtones (IGR : 1) favorable à une activité biologique plus importante (COT : 0,29\%). Malgré sa faible épaisseur, cet horizon à la morphologie «boudinée » a pu être observé dans toutes les coupes des deux secteurs et représente un niveau repère pour la localisation sur le terrain de l'industrie paléolithique N1 sous-jacente. Sa préservation indique l'absence de phase érosive à son sommet et une dynamique globalement en faveur de l'accumulation et de la sédimentation loessique qui s'affirme ensuite avec le dépôt de l'unité 8 de loess, elle aussi présente dans tout le secteur étudié. En conclusion, le développement du gley de toundra (unité 9), associé à un niveau de petites fentes de gel (F-6), confirme l'humidité stationnelle et des conditions initialement rigoureuses (mais sans pergélisol), suivies d'un bref épisode d'amélioration climatique accélérant la saturation en eau lors de la fonte estivale.

L'unité 8, observée dans tous les profils, traduit une intensification très nette de la dynamique éolienne avec la mise en place des premiers loess typiques carbonatés. Les datations OSL obtenues dans ce dépôt montrent des différences importantes $(61,7 \pm 4.0$ ka dans Hav.2-P5 et 48,7 \pm 2.9 ka dans Hav.1-P1). Cependant, la date obtenue à partir du profil P1 est fortement sujette à caution car elle est la seule à ne pas apparaître dans l'ordre stratigraphique (voir partie 2.4.1). Par ailleurs, même en prenant des précautions maximales, la probabilité de rencontrer un terrier colmaté par du matériel plus récent n'est pas nulle dans ce dépôt qui en comporte beaucoup. Pour ces raisons, nous retenons plus logiquement la date la plus ancienne qui situe ce loess au sein du Pléniglaciaire inférieur et qui est cohérente avec les résultats OSL qui l'encadrent.

D'une manière générale, la série des unités 12 à 8 semble correspondre à une phase très courte $(\sim 4-5 \mathrm{ka})$ qui place les dépôts contenant l'industrie lithique du niveau Hav.2-N1 (unité 12) vers 65 ka environ. La comparaison avec les dates TL (beaucoup plus anciennes) effectuées sur des silex chauffés retrouvés dans à la base de ce niveau suggère (comme la sédimentologie) qu'une partie du matériel provenant de l'unité 12 dérive du remaniement de niveaux archéologiques plus anciens.

En ce qui concerne cette partie inférieure de l'enregistrement d'Havrincourt (sous-séquences II-1 et II-2), une comparaison à plus longue distance avec les coupes du Pléistocène de Belgique comme Harmignies, Romont ou Remicourt (Haesaerts \& Van Vliet, 1973 ; Haesaerts et al., 1981, 1999 ; Frechen et al., 2001 ; Juvigné et al., 2008) débouche sur la mise en évidence de fortes analogies. En effet, dans ces trois profils, le complexe de sols humifères du Début Glaciaire (sols gris forestiers et horizon steppique polyphasé) est systématiquement recouvert par une épaisse unité discordante de limons lités hétérogènes remaniant les dépôts sous-jacents, coiffée par un horizon brun homogène (Sol de « Malplaquet »). Les premiers loess calcaires typiques incluant de petits gleys de toundra et datés de 66 à 60 ka (Frechen et al., 2001), apparaissent toujours au-dessus de cette unité. Selon ce schéma, le loess calcaire de l'unité 8 d'Havrincourt, qui correspond au premier marqueur loessique véritable de la séquence, pourrait être mis en parallèle avec celui les loess calcaires du Pléniglaciaire inférieur d'Harmignies et de Nussloch datés d'environ $65 \mathrm{ka}$ (Antoine et al., 2001, Frechen et al., 2001). 
Compte tenu des résultats des datations OSL, des observations de terrain (érosion importante, colluvionnement, fentes de gel) et de la sédimentologie (dynamique éolienne croissante) les unités 13 à 9 se rattachent aux phases initiales du Pléniglaciaire inférieur ; la mise en place des loess de l'unité 8 signant une phase plus avancée et plus intense de cette période.

\subsection{3 - Sous-séquence II-3 (unités 7 à 6a) : complexe de sols d'Havrincourt Pléniglaciaire moyen (PGM $\sim$ 55-35 ka)}

La partie supérieure des loess de l'unité 8 est ensuite affectée par une franche décarbonatation (LD-2) liée au développement du sol brun boréal sus-jacent de l'unité 7 (horizon limono-argileux compact, non calcaire brun orangé à structure polyédrique à lamellaire). Le développement pédologique de l'unité 7 est souligné par l'augmentation des pourcentages d'argile et de COT qui atteignent respectivement 28 et $0,5 \%$, et la décarbonatation et la forte dynamique de précipitation ferromanganique (enduits sur biotubules et concrétions). Cette unité correspond à un horizon $\mathrm{Bw}$ de sol brun boréal, qui possède les caractéristiques du sol principal (sol inférieur) du complexe du type Sol de SaintAcheul / Villiers-Adam (Antoine et al., 2003b), du sol des Vaux en Belgique (Haesaerts et al., 1981) et du sol de Gräselberg de la séquence de référence de Nussloch (Antoine et al., 2009).

L'enregistrement de Nussloch montre cependant que, dans les contextes les plus favorables à la sédimentation, ce sol peut se subdiviser en deux sous-unités, GBL et GBU, développées entre $\sim 58$ et $50 \mathrm{ka}$. Les datations OSL de $51,1 \pm 6,6$ ka et $46,6 \pm 5,8$ ka obtenues respectivement à la base et au sommet du sol de l'unité 7 confirment son attribution au Pléniglaciaire moyen et plus précisément à l'intervalle compris entre les interstades GIS 17 et 12. Selon cette interprétation, les différents épisodes froids situés vers 48 et 57 ka dans NGRIP n'auraient pas eu d'impact sur les environnements loessiques du nordouest de la France en raison d'une dynamique loessique trop faible pour permettre une séparation des différentes phases d'altération et de pédogenèse.

Par ailleurs, l'unité 7 se distingue par un grand nombre de terriers ovalaires attribuables à des mammifères fouisseurs (marmottes, spermophiles, arvicolidés, cf. partie 4) qui indique la présence d'une biomasse végétale beaucoup plus importante que lors du dépôt des loess sous-jacents. On remarque que ces structures, qui descendent profondément jusque dans le loess de l'unité 8 , apparaissent dans la même position stratigraphique dans plusieurs séquences du nord de la France (Antoine et al., 2003a), et plus généralement dans les autres profils du nord-ouest européen comme à Nussloch (Allemagne). L'abondance de ces terriers traduit le développement d'un environnement steppique singulier et particulièrement favorable au développement de ces rongeurs (surface stabilisée et densément végétalisée) qui, d'après les âges ${ }^{14} \mathrm{C}$, se placerait vers 45-46 ka au niveau de l'interstade GIS 12.

Dans cette hypothèse, la disparition brutale de ces rongeurs et leur piégeage en profondeur dans le réseau de terriers pourrait résulter d'un (ou de plusieurs) événements froids particulièrement intenses avec pergélisol, directement postérieurs (entre $\sim 44$ et $40 \mathrm{ka}$ ). Cet horizon de sol (unité 7) est en effet affecté par une fine structuration lamellaire liée aux multiples alternances gel-dégel postérieures au développement pédologique et indiquant au moins un épisode de développement de pergélisol (fig. 4 à 7).

Cette observation est renforcée par la mise en évidence d'un premier réseau de grandes fentes en coin de type « ice-wedge » ( 1,5 $\mathrm{m}$ de profondeur) qui s'ouvre au sommet de cette unité (F-5). Ces structures sont malheureusement assez mal conservées et souvent largement masquées par l'ouverture des grandes fentes du niveau principal F-4 qui s'y emboîtent systématiquement (fig. 6, 7 et 19). Compte tenu des âges OSL et de la succession

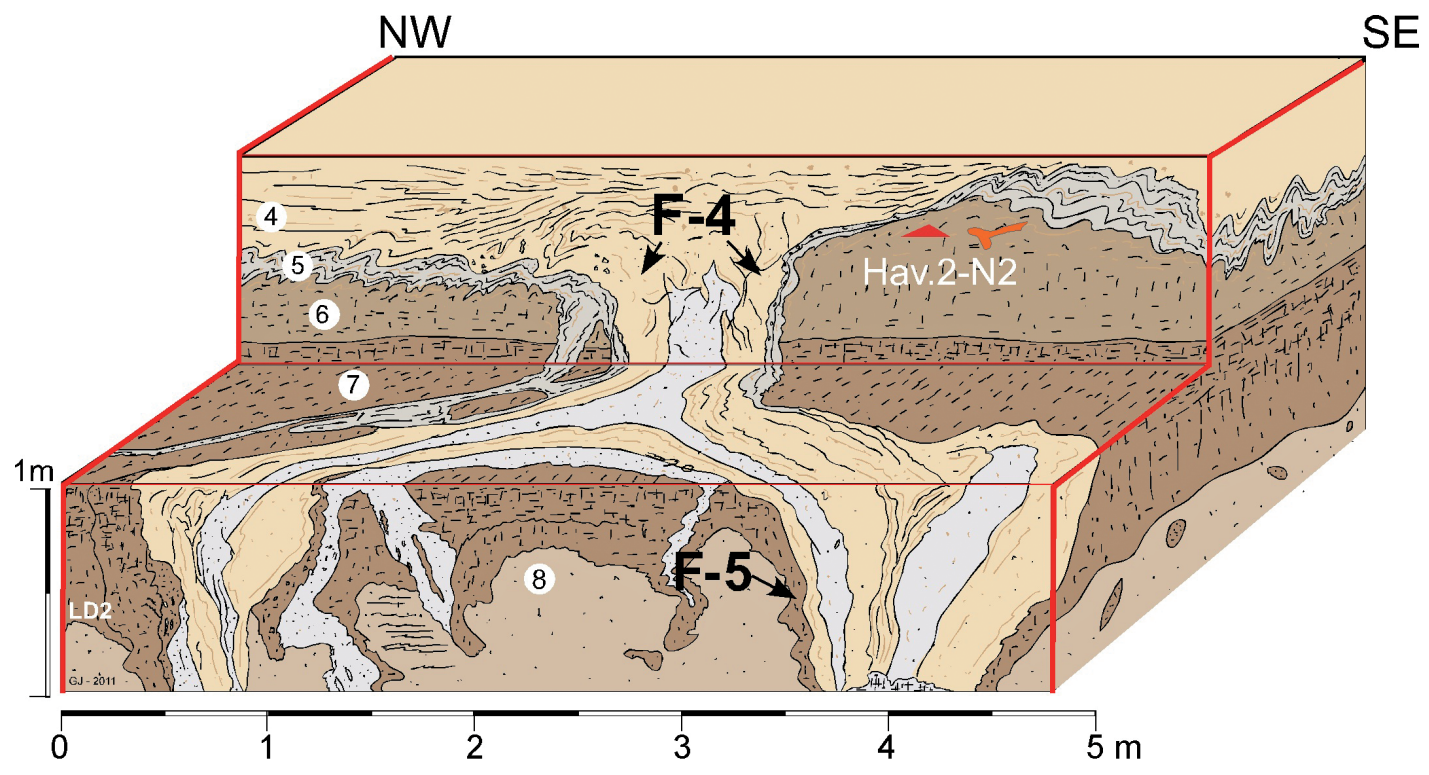

Fig. 19 : Hav.2-P1 : bloc diagramme illustrant une intersection dans le réseau de grandes fentes en coin F-4.

Fig. 19: Hav.2-P1: block-diagram showing an intersection in the network of large ice-wedge casts F-4. 

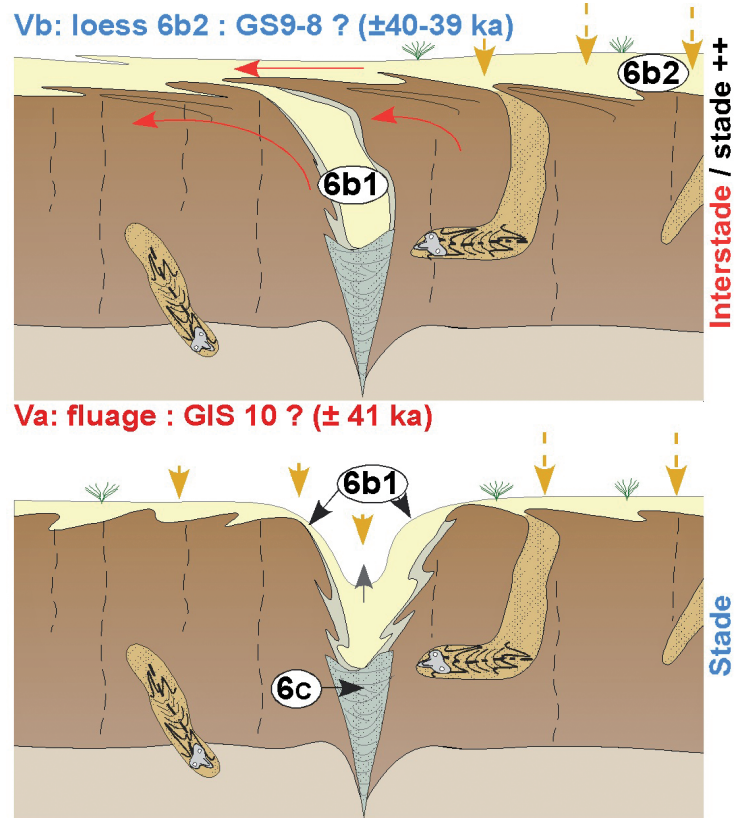

IV) loess $6 \mathrm{~b} 1 \mathrm{GS} 10$ ? ( $\pm 42 \mathrm{ka})$

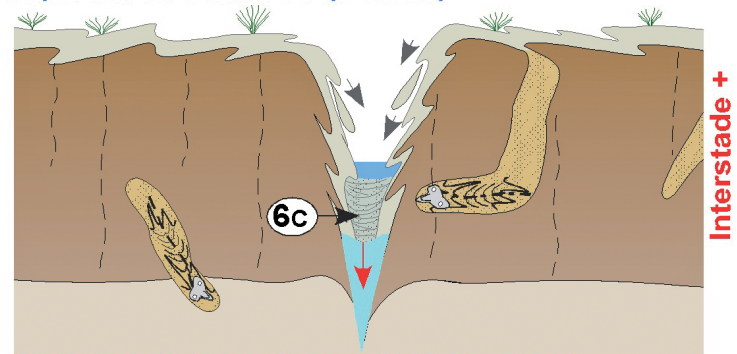

III) Dégradation du PF : GIS 11 ( \pm 43 ka)

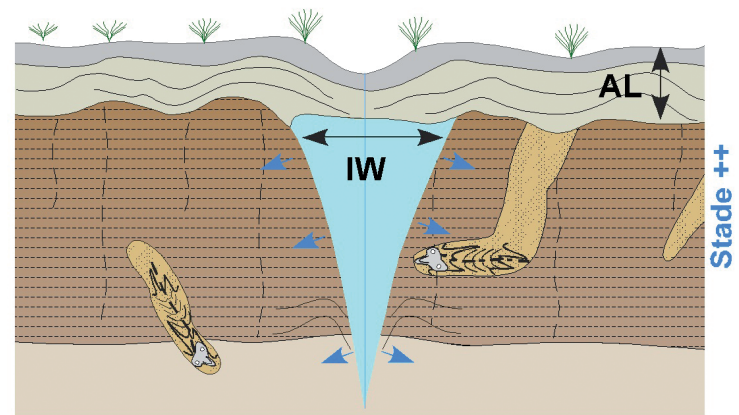

II) Permafrost / fentes F-5 : GS 11 ( \pm 44 ka)

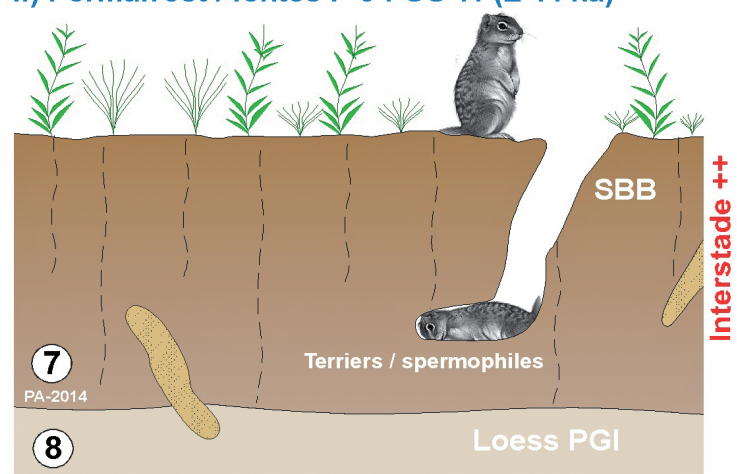

I) Phase finale sol brun 7 : GIS 12 ( \pm 45-46 ka)

Fig. 20 : Essai de reconstitution de l'évolution pédosédimentaire et paléoenvironnementale contemporaine de la partie inférieure du complexe de sols d'Havrincourt (première moitié du Pléniglaciaire moyen). PGI : Pléniglaciaire inférieur, IW : ice-wedge, AL : couche active, SBB : sol brun boréal.

Fig. 20: Attempt in reconstruction of the pedosedimentary and palaeoenvironmental evolutions contemporaneous of the lower part of the Havrincourt soil complex (first half of the Middle Pleniglacial). PGI : Lower Pleniglacial, $I W$ : ice-wedge, $A L$ : active laver, $S B B$ : boreal brown soil. des phases pédosédimentaires observées dans les unités qui l'encadrent, il est possible que ce premier réseau de grandes fentes de type " ice-wedge » se soit développé vers 44-43 ka au cours du GS 11. Ces structures ne seraient donc pas contemporaines de l'événement de Heinrich 4 qui se marque pourtant par des conditions particulièrement froides au niveau de l'Atlantique Nord vers $40 \mathrm{ka}$ (Bond et al., 1993 ; Marcott et al., 2011).

Le colmatage de ces structures est d'abord constitué par des limons à litages sub-horizontaux (alternances de lits bruns à nodules de sol de l'unité 7 remaniés et de lits loessiques plus clairs (unité 6c) qui traduisent un épisode de fonte des coins de glace et de ruissellement dans le réseau chenalisé ainsi créé (fig. 20). Cet épisode signe une déstabilisation intense du pergélisol (structures de fusion SF2), que l'on peut attribuer à une phase de réchauffement climatique rapide au cours du Pléniglaciaire moyen, vers 42-43 ka (interstades GIS 11 ?). A la suite de cet épisode probablement très court (cf. analogues actuels), un remplissage terminal de loess calcaires homogènes finit de colmater les structures dans un environnement qui redevient aride et froid (unité $6 b$ ). Une nouvelle phase de fluage par cryoreptation provoque ensuite un fauchage intense de ces structures et débouche localement sur leur fermeture par une « lèvre » de matériel argileux géliflué de l'unité 7 remanié (fig. 20) (GIS 10 ?). Elle est suivie d'une dernière phase de dépôt loessique peu épais antérieur à la formation du complexe de l'unité $6 \mathrm{a}$.

D'après leur position dans la stratigraphie et les données OSL, ces structures de fusion faiblement développées pourraient correspondre à celles décrites au sommet de l'unité 5 du complexe de sols de VilliersAdam (Antoine, 2003b). Leur mise en évidence à Havrincourt, en association avec un réseau de grandes fentes de type « ice-wedge », est très importante car elle confirme l'enregistrement d'un épisode de pergélisol au sein du complexe de sols du Pléniglaciaire moyen. Compte tenu de l'âge de la surface à partir de laquelle elles se sont ouvertes et de celui des dépôts sus-jacents, elles pourraient se rattacher à la péjoration climatique directement antérieure à GIS 11 vers $44 \mathrm{ka}$ (fig. 20 et 21). Le hiatus sédimentaire entre les unités 7 et $6 a$ est par ailleurs souligné dans les données analytiques par une chute des teneurs en carbone organique total $(0,32 \%)$ et un premier pic de $\mathrm{CaCO}_{3}$ indiquant une nouvelle phase d'apports loessiques à la base de l'unité 6. L'ensemble de l'unité 7 correspondrait donc au bilan de la partie la plus importante du Pléniglaciaire moyen et à l'intervalle $\sim 58-45$ ka (fig. 21). Néanmoins la structure de la courbe des argiles (croissance régulière interrompue brutalement à la transition des unités 7-6a) laisse supposer que l'horizon de l'unité 7 a subi une troncature importante en liaison avec la phase de dégradation du pergélisol associé au réseau de fentes F-5.

La formation de la partie supérieure du complexe de sol d'Havrincourt débute par un nouvel apport loessique (unité 6b) peu épais (essentiellement piégé dans les fentes du niveau F-5) suivi d'au moins deux phases de pédogenèse dont le degré de développement reste cependant nettement moindre que celui du sol de l'unité 7 . 
Havrincourt

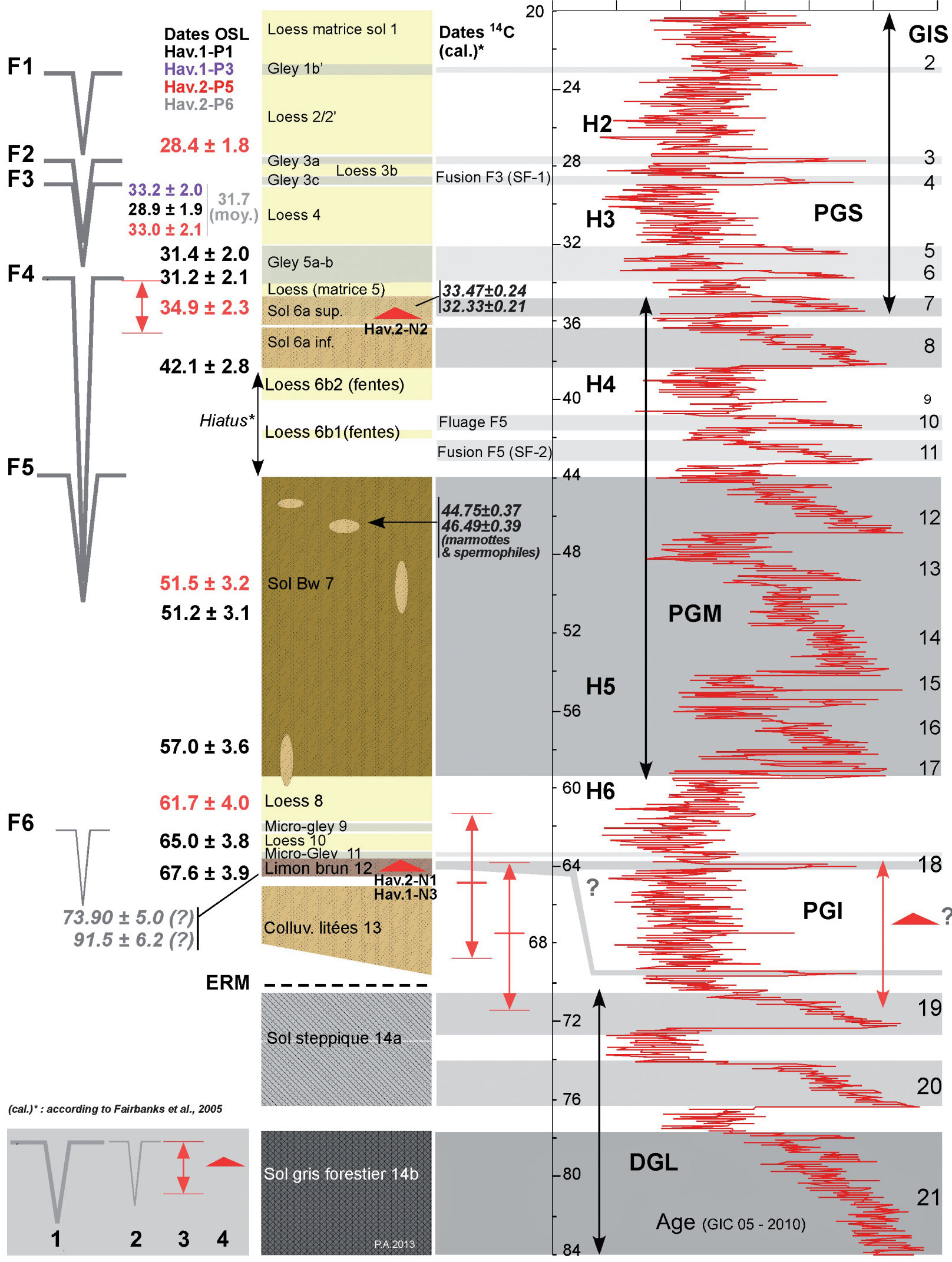

Fig. 21 : Proposition de corrélation entre la succession des différentes unités pédo-sédimentaires de la séquence synthétique d'Havrincourt et l'enregistrement paléoclimatique de North-GRIP, et datation des structures périglaciaires et des deux niveaux archéologiques principaux.

1/ Grandes fentes de type « ice-wedge », 2/ Fentes de gel, 3/ Intervalle chronologique des datations OSL de l'unité 12 contenant le niveau archéologique du Paléolithique moyen, 4/ Niveaux paléolithiques.

Fig. 21: Correlation proposal between the various pedosedimentatry units of the summarised Havrincourt sequence and the palaeoclimatic record of North-GRIP, and dating of the two main periglacial features and of the archaeoligical levels.

1/ Large ice-wedges, 2/ Frost cracks, 3/ Interval of the OSL datings from unit 12 that contains the Middle Palaeolithic archaeological level, 4/ Palaeolithic levels. 
Cette unité $6 \mathrm{a}$, distinguée sur le terrain par son faciès argileux compact à forte structure lamellaire et enduits de manganèses et d'oxydes de fer orangés, correspond à un horizon de type sol brun arctique à sol de prairie arctique (COT : 0,4 \%, argile : $29 \%$ ) hydromorphe et fortement structuré par les processus de gel-dégel. La structure en deux « pics » de la courbe du pourcentage en argile, ainsi que la présence d'une surface archéologique (niveau Hav.2-N2) dans le tiers supérieur de l'unité 6a suggèrent que cette dernière correspond à un ensemble polyphasé d'au moins deux horizons de sol superposés. L'originalité de cette unité est de présenter une augmentation progressive des pourcentages de $\mathrm{CaCO}_{3}$ de la base au sommet (2 à $8 \%$ ). Cette évolution, qui va à l'encontre de ce qui est généralement observé dans le cas d'un sol développé à partir de la surface d'un loess calcaire préalablement déposé, indique que l'on se situe dans un contexte où une altération pédologique peu intense est progressivement « noyée » sous des apports loessiques de plus en plus importants (période de transition Pléniglaciaire moyenPléniglaciaire supérieur).

Compte tenu des dates OSL et ${ }^{14} \mathrm{C}$ obtenues sur les restes de grands mammifères associés au niveau archéologique inclus dans sa partie sommitale ( 33-34 ka cal. BP), cet horizon pourrait représenter le bilan de la succession des interstades GIS 8 et 7 ou 8 à $6(\sim 38,5-33 \mathrm{ka})$. Dans cette configuration, les courtes périodes de péjoration climatique qui séparent ces interstades n'ont vraisemblablement pas été marquées dans les séquences d'Havrincourt par des phases de dépôts loessiques suffisamment importantes pour être préservées en tant qu'unités indépendantes (de la même manière que pour la période 45-58 ka).

D'après les données disponibles, cet horizon peut être corrélé avec la partie sommitale du complexe de SaintAcheul Villiers-Adam (sol brun supérieur) et le sol de Lohne des profils allemands comme Nussloch (Antoine et al., 2003c) attribués au complexe interstadiaire de Denekamp. Il ne semble pas avoir d'équivalent nettement individualisé dans les coupes de Belgique (télescopage au sommet du sol des Vaux ?).

Les données d'Havrincourt sont ici très importantes car elles apportent des informations fondamentales pour la datation et l'interprétation de cet horizon de sol qui, dans toutes les coupes loessiques européennes, marque la fin du Pléniglaciaire moyen et dont la datation fait l'objet d'un débat très actuel (Antoine et al., 2009, Kadereit et al., 2013).

D’une manière plus générale la question de la limite entre Pléniglaciaire moyen-Pléniglaciaire supérieur est importante et tout particulièrement en ce qui concerne Havrincourt où un niveau daté du Paléolithique supérieur ancien (Hav.2-N2) se situe au niveau de cette charnière.

Sur le terrain, cette limite stratigraphique sert de repère pour les corrélations à l'échelle européenne et il est important d'essayer de la préciser. D'une manière générale, elle se traduit par le passage brutal entre une longue période ( $20 \mathrm{ka})$ dominée par un très faible bilan de la sédimentation loessique $(0,05$ à $0,1 \mathrm{~mm} / \mathrm{an} !)$ et la formation de sols « bruns » de type sols boréaux à un environnement définitivement dominé par la mise en place des grandes couvertures de loess calcaires caractérisées par des taux de sédimentation très importants et un volume de dépôt sans précédent ( $\geq 1 \mathrm{~mm} / \mathrm{an}$, Frechen et al., 2003 ; Antoine et al., 2009). Cependant les enregistrements loessiques n'étant jamais continus, un hiatus variable, qui peut atteindre plusieurs milliers d'années ( $\geq 5 \mathrm{ka}$ ), caractérise cette limite. En effet, compte tenu des données chronologiques obtenues à Havrincourt et sur les séquences de références comme celle de Nussloch, l'explosion des taux de sédimentation loessique débuterait vers 32-30 ka alors que les derniers sols seraient datés de $~ 35-38 \mathrm{ka}$.

La préservation de l'unité 6 d'Havrincourt, qui correspond à une partie du bilan pédosédimentaire est exceptionnelle pour le nord de la France. L'unité 6 se place donc dans cette charnière qui marque l'extrême fin du Pléniglaciaire moyen. Par comparaison avec des séquences comme celles de Nussloch, cette unité polyphasée devrait être rattachée au PGM « final » pour sa partie inférieure (sol de Lohne) et au PGS « initial» pour sa partie supérieure plus hydromorphe et contenant le niveau du Paléolithique supérieur ancien N2 (Nussloch G1 ?). Néanmoins, le bilan étant très peu dilaté à Havrincourt, et pour des raisons évidentes de lisibilité sur le terrain, nous garderons l'ensemble de cette unité au sein du Pléniglaciaire moyen. Enfin il faut signaler qu'un niveau du Paléolithique supérieur ancien daté de $28680 \pm 200 \mathrm{BP}(\sim 34 \mathrm{ka}$ cal. BP) a été découvert récemment dans la séquence loessique de Renancourt près d'Amiens au sein d'une unité de limons brunâtres antérieure à la mise en place des loess du Pléniglaciaire supérieur et montrant un faciès similaire à l'unité $6 \mathrm{a}$ d'Havrincourt (Paris et al., 2013).

La composition des malacofaunes du Pléniglaciaire moyen du nord de la France étant quasiment inconnue faute des données dues à la décarbonatation quasi-généralisée des sols bruns boréaux de cette période, elle ne peut fournir d'éléments susceptibles de contribuer à préciser la chronostratigraphie de l'unité 6. Les proportions des différentes espèces sont néanmoins similaires à celles de la malacofaune extraite du paléosol supérieur du complexe de sols du Pléniglaciaire moyen à Languevoisin (Moine, 2011).

Les proportions des taxons de chaque profil indiquent que l'unité 6a est associée à un milieu découvert à végétation pauvre, plutôt sec sur Hav.1-P1 et plus humide sur Hav.2-P5. Cependant, les taux de carbonate étant similaires dans les deux profils, la forte différence d'abondance totale entre les deux loci pourrait s'expliquer par des différences topographiques induisant un milieu localement plus humide et donc plus favorable au développement des mollusques sur Hav.2-P5. Toutefois, on note d'une part que l'enregistrement malacologique sur Hav.2-P5 ne débute qu'au milieu de l'unité 6, et d'autre part une augmentation d'abondance beaucoup plus abrupte à la transition des unités 6a-5 sur Hav.1-P1 que sur Hav.2-P5, le changement dans les proportions n'étant qu'à peine plus marqué sur Hav.1-P1. Ce faisceau de 
critères associé aux déformations périglaciaires affectant la partie supérieure de l'unité 6a suggère qu'elle ait pu être partiellement érodée sur Hav.1-P1. Par conséquent les deux enregistrements malacologiques seraient diachroniques, celui de Hav.2-P5 succédant chronologiquement à celui de Hav.1-P1.

Ainsi, les malacofaunes de l'unité 6a reflètent-elles un milieu plutôt froid, sec et ouvert à végétation peu diversifiée sur Hav.1-P1, devenant progressivement plus humide sur Hav.2-P5. Cette tendance est pour tout ou partie renforcée au sommet de l'unité sur Hav.2-P5 par la présence de fentes en coin remplies du matériel gleyifié de l'unité sus-jacente et qui n'ont pu être évitées lors de l'échantillonnage. En admettant l'attribution de l'unité 6a à la fin du Pléniglaciaire moyen, l'environnement partiellement dénudé décrit à Havrincourt contrasterait fortement avec la végétation de steppe / toundra à toundra arbustive plus ou moins diversifiée reconstituée dans la vallée du Rhin : Achenheim (Puisségur, 1978), Remagen (Schiermeyer, 2002), Nussloch (Moine et al., 2005).

\subsection{4 - Sous-séquence II-4 (unités 5 à 2) : Pléni- glaciaire supérieur (PGS 30-17 ka)}

A la suite de la discussion précédente concernant la limite Pléniglaciaire moyen-Pléniglaciaire supérieur, la première unité rattachée au Pléniglaciaire supérieur est donc le gley de toundra de l'unité 5 qui représente une limite fondamentale au regard du complexe de sols sousjacents. En effet, indépendamment de son faciès lié à l'hydromorphie, cette unité possède dès sa partie basale, des caractéristiques sédimentologiques de type loessiques ( $\mathrm{COT}$ faible, $\mathrm{CaCO}_{3}$ élevé) qui indiquent qu'elle s'est développée sur un apport éolien « frais » distinct du limon loessique argileux altéré et partiellement hydromorphe du sommet de l'unité 6a. Cet horizon de gley de toundra cryoturbé (fig. 11-C et E) résulte de la saturation saisonnière en eau de la couche active d'un pergélisol. Il est jalonné par une bande irrégulière à faciès laminé de quelques centimètres d'épaisseur (unité 5a), totalement déferrifiée, enrichie en $\mathrm{CaCO}_{3}$ secondaire (redistribution de carbonates sous la forme de petites concrétions et manchons racinaires) et en débris organiques noirâtres (restes de végétation, croûte cryptogamique ?) (fig. 11-H). Un pic marqué des valeurs de COT et une augmentation de la susceptibilité magnétique sont associés à ce niveau et pourraient signaler un dédoublement de l'unité 5. Dans les deux secteurs, les courbes de l'abondance malacologique de l'unité 5 se caractérisent par deux pics successifs d'intensité décroissante. Cette observation va aussi dans le sens d'un gley complexe dédoublé.

Ces deux pics d'abondance accompagnés d'augmentations de la proportion en individus juvéniles chez le genre Pupilla indiquent un réchauffement et un allongement de la saison chaude (été et saisons de transition), cohérents avec la fusion et la cryoturbation du gley de toundra caractérisant des interstades (Moine et al., 2008). Les proportions croissantes des taxons hygrophiles et palustres associées à ces deux pics soulignent aussi des conditions plus humides. Cette présence plus importante de l'eau au sommet des gleys de toundra induit en outre les taux de fractionnement des coquilles du genre Pupilla les plus élevés en association avec les pics d'abondance. Enfin, les abondances moindres et les proportions plus importantes en taxons hygrophiles et palustres reflètent des conditions environnementales moins clémentes et plus humides durant le pic sommital dans chacun des deux loci. Ces observations sont identiques à celles réalisées dans les gleys de toundra de Bourlon et G2 de Nussloch (Moine et al., 2011, Moine, 2014). A Nussloch, le biphasage associé du gley de toundra G2 a été démontré grâce au profil P4 (Antoine et al., 2002b) alors qu'à Bourlon il n'est comme à Havrincourt fondé que sur des éléments malacologiques. La phase stadiaire censée séparer ces deux interstades n'est en outre clairement exprimée dans aucun de ces trois sites. Par ailleurs, la composition de la malacofaune ne reflète aucun accroissement de la diversité végétale, à moins que les zones refuges des mollusques n'aient été trop éloignées pour permettre une recolonisation de la région par de nouvelles espèces durant ces brèves phases de réchauffement (Moine et al., 2008).

Les datations OSL, qui situent l'unité 5 autour de 31-32 ka (31,2 $\pm 2,1$ ka et $31,4 \pm 2,0 \mathrm{ka})$, sont cohérentes avec les dates des niveaux sous-jacents, les âges ${ }^{14} \mathrm{C}$ du niveau archéologique Hav.2-N2 ainsi qu'avec une attribution à l'interstade GIS 5 ou 5-6 ( 32-33,5 ka). L'âge de l'unité 5 d'Havrincourt est également cohérent avec celui du gley de toundra G2 de Nussloch (Antoine et al., 2009) et très probablement avec celui de Bourlon (Moine et al., 2011). Le biphasage mis en évidence dans ces trois sites caractériserait donc une réponse généralisée des paléoenvironnements ouest-européens à des phases de réchauffement millénaires du climat dans un contexte de faible taux de sédimentation loessique (Moine et al., 2011).

La suite de la sous-séquence Pléniglaciaire supérieur s'individualise d'une manière très nette avec la mise en évidence d'une nouvelle péjoration climatique particulièrement intense qui se traduit par la formation d'un réseau de grandes fentes à coins de glace de type « ice-wedge » ( 2 à $3 \mathrm{~m}$ de profondeur / ouverture $\sim 0,5 \mathrm{~m}$ ) à maille décamétrique (12-14 m). Ce réseau polygonal (F-4, fig. 11-A et $\mathrm{H}$ ) (polygones de toundra) dont l'extension horizontale a pu être suivie et photographiée par un drone d'une manière unique sur plus de $4000 \mathrm{~m}^{2}$ lors du décapage (fig. 11-B), représente la signature inédite d'un pergélisol continu qui constitue un marqueur du début Pléniglaciaire supérieur (fig. 10, 21). Ces structures sont particulièrement bien conservées à l'interface des unités 5-6 et 4 où leur visibilité est accentuée par les importants contrastes de lithologie et de coloration qui distinguent l'encaissant (limons brun grisâtre argileux) du remplissage de ces structures (loess calcaire beige clair). Sur la base des travaux menés dans l'Arctique (Pissart, 1987 ; Van Vliet-Lanoë, 1987 ; French, 2007 ; French \& Shur, 2010 ; Matsuoka, 2011), il a été montré que ces grandes fentes à coins de glace de type épigénétique résultaient de la répétition de la succession d'événements suivants : 
- A) fracturation verticale initiale de quelques millimètres de large, à partir du toit du pergélisol, lors d'une chute très brutale de la température. Les conditions pré-requises sont une couverture neigeuse peu épaisse (moins de $50 \mathrm{~cm}$ ) et un appauvrissement en eau par cryosuccion. Le régime thermique spécifique estimé pour la genèse de cette ouverture correspond à une température moyenne de l'air annuelle MAAT de $-7^{\circ} \mathrm{C}$ à $-9^{\circ} \mathrm{C}$ et une température de l'air au moment de la fissuration inférieure à $-25^{\circ} \mathrm{C}$. En revanche, la température au toit du pergélisol avoisinerait les $-10^{\circ} \mathrm{C}$. A Havrincourt substrat limono-argileux et une topographie plane à très faible pente sont particulièrement favorables au développement de ces structures;

- B) fonte printanière superficielle du pergélisol (couche active) entraînant un remplissage des fissures par les eaux de fonte immédiatement congelées au contact du sol gelé. La congélation de l'eau dans la fissure entraîne une augmentation de volume et un élargissement de la fissure sous l'effet de la transformation de l'eau en glace;

- C) retour à l'étape A lors de l'hiver suivant avec nouvelle fissuration brutale guidée par la structure existante.

La formation de grandes fentes à coin de glace, dont l'ouverture atteint $\sim 0,5 \mathrm{~m}$ à Havrincourt, pourrait correspondre à la répétition d'environ 500 cycles annuels et donc indiquer un hiatus de $0,5 \mathrm{ka}$ dans la séquence. Néanmoins les vitesses annuelles d'ouverture de ces structures sont encore mal connues et liées en partie à la nature du substrat et à sa teneur en glace (Black, 1976 ; Murton, 2007).

A Havrincourt, le colmatage sédimentaire qui a permis la préservation de ces pseudomorphoses de fentes en « V » à la suite de la disparition des coins de glace est constitué par deux phases bien distinctes :

-1) recouvrement des bordures de la fente en cours de dégradation (fusion du coin de glace) par du matériel gleyifié grisâtre issu du fluage lent de la couche active du pergélisol (unité 5) (fig. 19, 20). L'absence de stratification dans le colmatage des fentes du réseau principal F-4 implique une décroissance lente et progressive du coin de glace avec drainage des eaux de fonte en profondeur sans ruissellement contrairement à ce qui a pu être observé au niveau du réseau de fentes F-5;

-2) colmatage loessique homogène en milieu aride et froid et fossilisation définitive de la structure.

Cette succession, qui a été étudiée en détail depuis plusieurs années dans les séquences du nord-ouest européen, traduit l'alternance rapide de deux phases climatiques extrêmes (Moine et al., 2008 ; Antoine et al., 2009) :

-1) développement du pergélisol riche en glace et d'un réseau de grandes fentes en coin lors d'une période caractérisée par des hivers extrêmement rigoureux susceptibles d'initier le processus. Le milieu est cependant assez humide pour que le sol soit riche en glace (neige) et la sédimentation éolienne est en forte réduction

- 2) dégradation rapide du pergélisol entraînant la fusion des coins de glace, l'approfondissement de la couche active (épaississement du faciès gley de toundra), puis son fluage dans les coins libérés par la fusion de la glace. En cas de réchauffement particulièrement intense et rapide, et en présence d'une pente significative, l'eau de fonte libérée par la fusion des coins de glace provoque des processus de ruissellement localisés (chenaux de fonte) qui érodent les structures en coin initiales (érosion thermique) (niveau F-2). Dans les contextes de versant, ce processus peut aboutir à l'élargissement progressif des chenaux initiaux guidés par la structure polygonale et la mise en place d'un réseau de drainage et d'érosion thermokarstique. Dans un stade ultime, la coalescence de ces chenaux provoque la formation de ravines thermokarstiques susceptible d'entailler profondément les versants comme cela a été observé à Villiers-Adam (Antoine et al., 2003b) ou à Nussloch (Antoine et al., 2001, 2009, 2013).

A Havrincourt la fossilisation du gley de toundra et du réseau de grandes fentes associé à un dépôt éolien (loess) intervient dans un milieu qui redevient très aride et froid. Néanmoins, l'imbrication des « langues » de gley géliflué de l'unité 5 et du loess sus-jacent (unité 4) montre la rapidité du passage entre la phase de fluage finale du gley de toundra et la mise en place des premiers dépôts loessiques, mais aussi la poursuite des cycles annuels de gel-dégel qui continuent de déstabiliser les horizons superficiels du sol.

La péjoration climatique se poursuit avec la mise en place des loess calcaires homogènes de l'unité 4 (IGR : 1,65; Argile : $17 \%$; COT : 0,16\%) sur une épaisseur particulièrement constante dans les deux sites $(\sim 0,6$ à $0,8 \mathrm{~m})$. Cette unité est localement affectée par une décarbonatation (LD-1) liée au sol de surface dans le secteur 1 où les dépôts sus-jacents sont moins épais (unité 4').

Ces loess, qui ont été observés dans la même position stratigraphique dans un grand nombre de profils du nord de la France, notamment le long du tracé du TGV Nord (Antoine, 1991), constituent un « marqueur » du début du Pléniglaciaire supérieur dont la datation était jusqu’à maintenant assez imprécise (Locht et al., 2006). Les données d'Havrincourt et les dates OSL nous permettent maintenant de les placer en parallèle avec la péjoration climatique majeure centrée sur $30,5 \mathrm{ka}$ dans les enregistrements paléoclimatiques de référence (fig. 21). On remarque par ailleurs que cette période correspond à l'événement de Heinrich 3 décrit dans les carottes marines de l'Atlantique Nord (Bond et al., 1993, Marcott et al., 2011). Comme dans le cas du réseau F-5, la formation du pergélisol associé au réseau majeur de coins de glace F-4 serait antérieure de quelques milliers d'années à l'événement de Heinrich 3.

Les analyses d'anisotropie de la susceptibilité magnétique menées sur toute l'épaisseur de cette unité débouchent sur la mise en évidence d'une linéation magnétique dont les variations sont corrélées aux valeurs des indices granulométriques (fig. 14 et 16).

Il est très probable que l'intensification de la dynamique éolienne enregistrée par l'IGR et l'U-ratio soit accompagnée d'une augmentation des vitesses moyennes des vents qui permet le développement d'une linéation 
magnétique. Les mesures indiquent cependant une direction dominante NNE / SSO, en net désaccord avec les orientations NNO-SSE généralement admises pour le dépôt des loess weichseliens de la zone ouest européenne (Lautridou, 1987 ; Antoine et al., 2009). Cette première étude, très prometteuse, devra être complétée par l'analyse des autres phases de dépôt loessique du PGS afin de rechercher d'éventuels changements majeurs de la direction moyenne des vents dominants au cours de cette période. Ces données apparaissent en effet fondamentales pour la modélisation de la circulation atmosphérique et de ses relations avec la sédimentation éolienne dans l'ouest de l'Europe au cours du Dernier Glaciaire (Sima et al., 2013).

Le sommet de cette unité loessique est matérialisé par un gley de toundra épais et complexe qui se subdivise en deux sous-horizons séparés par un mince dépôt de loess calcaire essentiellement préservé dans le profil Hav.2-P6 (fig. 7) au sein d'une pseudomorphose de coins de glace (réseau F-3). Le remplissage de cette fente F-3 montre, dans le profil $\mathrm{P} 3$ du secteur 1 , des lits à stratifications entrecroisées (SF1) matérialisant un épisode de fusion du pergélisol associé lors d'un épisode de réchauffement (interstade). Cette bipartition du gley 3, souvent difficile à observer sur le terrain en l'absence de structure en coin intermédiaire, est cependant bien enregistrée dans les données analytiques, notamment par les argiles, le COT et la malacologie. Bien que moins net que dans l'unité 5 , un profil bimodal concernant tous les taxons caractérise la courbe d'abondance totale en mollusques du gley de l'unité 3. Les malacofaunes, fortement dominées par le taxon hygrophile $S$. oblonga, reflètent un milieu humide. Dans les deux pics d'abondance, les proportions plus élevées du taxon palustre $P$. alpicola soulignent une accentuation de l'humidité. Malgré tout, la végétation semble aussi peu diversifiée que pour l'unité 5. Les réserves émises alors à ce sujet s'appliquent également ici. Il est donc fort probable que l'unité 3 soit aussi biphasée et qu'elle résulte d'une succession de deux interstades. Même si l'environnement associé au pic supérieur semble légèrement moins humide que celui du pic inférieur, leur différentiation est beaucoup moins évidente que pour ceux de l'unité 5 , notamment en raison de la forte cryoturbation de l'unité 3.

L'horizon supérieur du gley de toundra (unité 3a) est associé au niveau de grandes fentes (réseau F-2) à colmatage loessique homogène de $\sim 0,3 \times 1,2 \mathrm{~m}$ de profondeur qui s'emboîtent systématiquement dans le précédent. Ces fentes, moins déformées que celles du niveau F-3 (sans structures de fusion), sont fossilisées par un dépôt de loess calcaire homogène (unité 2). La succession des faciès $3 a-b$ correspond à la même dynamique que celle qui a été mise en évidence pour les unités 4-3c et détaillée ci-dessus. La maille du réseau n'a pas pu être identifiée en détail en raison des conditions d'affleurement (absence de décapage horizontal) néanmoins on remarque que le niveau de fente F-2 semble s'emboîter systématiquement (comme d'ailleurs le niveau de fente F-3) dans le niveau de fente principal F-4, indiquant un réseau polygonal à maille décamétrique de la même ampleur. Une seule datation OSL $(28,4 \pm 1,8 \mathrm{ka})$ est disponible pour le loess qui fossilise ce niveau dans le profil Hav.2-P5. Cette date montre néanmoins la rapidité de la mise en place de la séquence représentée par la succession du loess de l'unité 4, du gley dédoublé de l'unité 3 et de la base du loess de l'unité 2 (fig. 21). En raison de sa proximité avec la base du sol de surface, ce loess est par contre souvent décarbonaté notamment sur le secteur 1 où il est un peu moins épais.

L'analyse de cette partie de la séquence montre donc des caractéristiques comparables à celles du gley complexe observé dans ces séquences du Nord et de la Somme (notamment sur le TGV-Nord) et dénommé « Horizon cryoturbé du Santerre » (Antoine, 1991). Lâge de cette partie est encore mal connu, néanmoins compte tenu du calage par les nouvelles datations OSL et de l'histoire climatique enregistrée dans cette partie de la série (succession rapide de deux interstades séparés par un stade), il est possible de les rapprocher de la succession des interstades GIS 4 et GIS 3 centrés respectivement autour de 27,4 et 28,5 ka. Dans l'Ouest européen, ce doublet de gleys de toundra a été mis en évidence et daté autour de 28 ka dans la séquence de référence de Nussloch (G3-G4, Antoine et al., 2009) et pourrait correspondre aux gley HC6 des séquences belges comme Harmignies, bien que les âges TL y soient un peu plus jeunes (âge moyen autour de $\sim 26 \mathrm{ka}$, Frechen et al., 2001). Notons que les malacofaunes des gleys de toundra G3 et G4 de Nussloch sont différentes de celles du gley de toundra G2, assez similaires entre elles et que les deux pics d'abondance associés sont à peu près de même de taille (Moine et al., 2008), ce qui leur confère une certaine ressemblance avec ceux de l'unité 3 d'Havrincourt même si la richesse $y$ est nettement moindre.

Dans les profils du secteur 2, un dernier horizon de type gley de toundra associé à de rares fentes en coin a été observé (niveau F-1). Contrairement aux niveaux sousjacents, celui-ci, complètement intégré au sein de l'horizon $\mathrm{Bt}$ argileux du sol de surface est souvent difficile à mettre en évidence. Cette configuration se rencontre dans de nombreux profils du nord de la France en raison de la faible épaisseur du loess terminal (1 à $2 \mathrm{~m}$ ) qui est alors totalement décarbonaté et illuvié (fig. 15). Il apparaît néanmoins dans le secteur 2 en plusieurs points du profil sous la forme de structures en $« \mathrm{~V} » \mathrm{de} \sim 0,3 \mathrm{~m}$ d'ouverture pour $\sim 70-80 \mathrm{~cm}$ de profondeur à colmatage brun plus grisâtre que l'encaissant. Par comparaison avec les structures mieux conservées et les données de la bibliographie, il s'agit d'un niveau de pseudomorphoses de grandes fentes de gel à colmatage loessique. Ce niveau n'apparaît pas associé à une interface stratigraphique, probablement en raison du masquage lié à la décarbonatation et aux processus d'illuviation associés au développement du sol de surface. Cependant, d'après les données granulométriques (IGR notamment), il semble bien se situer au sommet d'une phase d'accumulation loessique majeure et à la transition avec les derniers $0,5 \mathrm{~m}$ de loess caractérisés par un IGR, une médiane et un U-ratio nettement plus faibles (fig. 12 et 14). 
Aucune datation n'est disponible pour ce niveau, cependant, compte tenu de la séquence d'événements décrite dans les dépôts sous-jacents, il est possible que cet horizon se soit développé au cours du dernier interstade court de la fin du Dernier Glaciaire (GIS 2 vers $23 \mathrm{ka}$ ). Du point de vue stratigraphique, il pourrait correspondre à l'Horizon de Nagelbeek (Haesaerts et al., 1981), dont un équivalent a été récemment mis en évidence dans le nord de la France dans les coupes d'Onnaing (Valenciennes, chantier Toyota, Antoine, inédit), ou le gley G8 de Nussloch directement sus-jacent à un loess daté de 23 ka par OSL (Antoine et al., 2009).

\subsection{5 - Sous-séquence IV (unités 1 à 0 ) : Tardigla- ciaire et Holocène}

La séquence d'Havrincourt se termine avec la formation du sol brun lessivé de surface qui représente le bilan du Tardiglaciaire et de l'Holocène (Van Vliet-Lanoë et al., 1992). Il est représenté par :

- 1) un horizon à doublets (horizon B à illuviations silteuses à argilo-silteuses en bandes ondulées 1b) qui résulte d'une décarbonatation suivie d'une illuviation en bandes liée à la dégradation finale du pergélisol au début du Tardiglaciaire dès 14-15 ka;

-2) un horizon Bt argileux typique à revêtements argileux dont le développement se poursuit tout au long de l'Holocène (unité 1a). La position actuelle de la limite de décarbonatation, qui apparaît totalement déconnectée de la base de l'horizon $\mathrm{Bt}$, correspond vraisemblablement à une évolution très récente du sol de surface sous l'effet de la dénudation et des pratiques culturales (exposition du sol nu, reprise de lessivage). Cette limite, qui peut localement atteindre plus de $3 \mathrm{~m}$ de profondeur, affecte souvent une partie importante des loess les plus récents et souligne la base de structures récentes comme les tranchées de la Première Guerre Mondiale (1914-1918). Par comparaison avec d'autres profils régionaux, l'épaisseur assez faible de l'horizon Bt 1a à Havrincourt traduit une érosion anthropique responsable de l'ablation d'une tranche de sol d'au moins 0,2 à $0,3 \mathrm{~m}$. L'impact anthropique sur ce sol est nettement souligné par les très nombreux pédotubules à enduits argilo-silto-organiques sombres qui le pénètrent (agricutanes résultant du lessivage de la surface d'un sol dénudé par l'agriculture). Enfin, à Havrincourt, le sol de surface est souvent affecté par de profondes traces de remaniements (tranchées, obus de la bataille de Cambrai, etc.) qui résultent de l'activité de ligne de front de la Première Guerre mondiale (1914-1918).

\section{5 - LES OCCUPATIONS PALÉOLITHIQUES (E.G., D.H.)}

Les fouilles réalisées à Havrincourt ont révélé quatre niveaux d'occupation en place (N0, N1, N2, N3 dont deux majeurs N2 et N3) et dont les processus taphonomiques ont pu être pleinement cernés. Les données acquises représentent une occasion unique de pouvoir appréhender la dynamique d'occupation du territoire par les peuplements humains du Paléolithique moyen final et du Paléolithique supérieur ancien lors de la dernière glaciation.

\section{1 - TAPHONOMIE}

L'analyse taphonomique est un préalable indispensable à toute étude archéologique. Elle est le garant de l'interprétation du site. Etant donné l'étendue des surfaces décapées $\left(2000 \mathrm{~m}^{2}\right.$ pour le secteur 1 , et $4100 \mathrm{~m}^{2}$ pour le secteur 2), il est envisageable que la quasi-totalité des vestiges archéologiques ait été récoltée, bien que dans le cas du secteur 1, il est probable que les limites de l'occupation humaine s'étendaient au-delà de la zone de décapage. De nombreux niveaux paléolithiques ont été découverts dans les deux secteurs de fouille mais tous n'ont pas la même validité scientifique. En effet, certains ont subi de nombreuses perturbations post-dépositionnelles, d'autres peuvent être considérés comme n'ayant subi que très peu (de l'ordre de quelques centimètres au maximum) ou aucun déplacement. L'objectif est de discuter du caractère en place de chaque série mise au jour.

\subsection{1 - Hav.1-2010}

Du matériel lithique et de la faune ont été découverts dans trois unités stratigraphiques distinctes. Un seul des niveaux archéologiques a été jugé en position in situ pour les raisons suivantes (du bas vers le haut de la séquence).

Une première industrie lithique a été récoltée au sein d'un limon humifère attribuable au Début Glaciaire weichselien (unité 14). Ce limon est piégé dans des blocs de limon gris humifère remaniés localement associés à des dépôts rougeâtres issus de la dégradation de l'horizon du Bt Eemien. D’une manière générale, ces dépôts sont remaniés sous forme de blocs gelés ou piégés dans des structures de dissolution en puits. Certaines pièces de l'industrie sont patinées, d'autres sont particulièrement émoussées. Ce dépôt hétérogène découle du démantèlement du sol humifère sur le haut du versant et de son remaniement local par colluvionnement et ruissellement. Les conditions de dépôts de ce matériel vont dans le sens d'un matériel lithique remanié. Ainsi, le matériel lithique (présence de lames attribuables aux industries du Début Glaciaire) est en place au sein des blocs de sol humifère. Cependant, ces derniers ont été remaniés et ne sont plus spatialement en position primaire.

Au sein des limons lités sus-jacents (unité 13), quelques artefacts ont été récoltés, certaines pièces sont lustrées (patine brillante). Ces limons témoignent de phénomènes de ruissellement intense et l'industrie présente n'est donc plus en position primaire. Les limons bruns dans lesquels est préservée l'industrie N3 se sont déposés en contexte loessique associé à des épisodes de colluvionnement (unité 12). Ce phénomène a eu pour conséquence un enfouissement rapide et relativement peu perturbateur du matériel archéologique. L'unité sédimentaire, homogène dans son ensemble, est présente sur quelques décimètres $(20-30 \mathrm{~cm})$ sur l'ensemble de l'emprise de fouille. 
Les pièces, éparses, ont systématiquement été retrouvées en position horizontale et leur état de fraîcheur est remarquable. L'industrie lithique et la faune qui lui est associée sont en position primaire (Hav.2-N3). Il ne semble y avoir eu aucune modification post-dépositionnelle, à l'exclusion de la dissolution probable de vestiges osseux.

Il est important de souligner que quelques restes fauniques ont été découverts dans l'horizon brun (unité 6) situé directement sous un gley. Aucun artefact lithique n'a été récolté. Au vu des observations faites par la suite dans le secteur 2, il est fort probable que ces restes osseux soient contemporains du niveau supérieur Hav.2-N2, dans la même position stratigraphique. Cependant, faute de liens directs sur le terrain entre les deux secteurs, il serait hasardeux d'affirmer leur stricte contemporanéité.

\subsection{2 - Hav.2 - 2011}

Du matériel lithique et de la faune ont été découverts dans six unités stratigraphiques distinctes. De la base au sommet de la stratigraphie, trois niveaux ont été jugés en place pour les raisons suivantes.

Les indices paléolithiques les plus anciens sont contenus dans des limons loessiques à doublets dont la limite sommitale est diffuse (unité 16). Le dépôt éolien a eu pour conséquence un enfouissement rapide et non perturbateur du matériel archéologique. Aucun élément ne va à l'encontre d'une position primaire du matériel (N0). Ces dépôts sédimentaires étant attribuables à la fin du Saalien, ces indices d'occupation sont d'autant plus intéressants qu'ils sont rares dans la région. Néanmoins, seuls quelques artefacts (deux éclats et une pointe Levallois) et trois os longs ont été découverts sur l'ensemble de la surface fouillée.

Un limon argileux, orangé, calcaire (unité 15a) interprété comme l'horizon attribuable à l'interglaciaire eemien, contient quelques pièces lithiques. Cet horizon est fortement dégradé et hétérogène dans sa structure. Les observations de terrain plaident en faveur du caractère secondaire de cette industrie.

Comme dans le cas du secteur 1, une industrie a été récoltée au sein d'un limon humifère attribuable au Début Glaciaire weichselien (unité 14). Ce dernier se présente sous forme de blocs cisaillés épars et se retrouve également piégé dans des structures de puits karstiques. Pour les mêmes raisons que celles évoquées ci-dessus, cette série n'est pas en position primaire.

Les limons bruns dans lesquels est préservée l'industrie Hav.2-N1 sont également présents (unité 12). L'industrie est conservée uniquement dans la partie ouest du secteur 2 sur environ $1500 \mathrm{~m}^{2}$. Dans la partie est, les industries lithiques recueillies au sein de l'unité 13 et à la base de l'unité 12 semblent issues du démantèlement d'occupations du Paléolithique moyen antérieures. Une attribution de ces artefacts au Début Glaciaire weichselien semble en concordance avec les datations obtenues. Ainsi, seul le matériel lithique et faunique en position primaire, clairement identifié dans le limon loessique brun sous le second gley (unité 12) est pris en compte dans l'analyse.
Quelques pièces éparses (silex et faune) ont été récoltées au sommet d'un limon argileux brun-orangé surmonté d'un cailloutis (unité 7) Cette unité est fortement marquée par des processus de gel/dégel ayant, entres autres, pour conséquence une redistribution du matériel. Ces quelques pièces sont en position secondaire.

L'industrie la plus importante numériquement se localise au sein d'un loess brun grisâtre homogène et faiblement calcaire (unité 6a) directement sous-jacent au gley de toundra de l'unité 5 (Hav.2-N2). Au-delà des observations stratigraphiques, la répartition du matériel lithique plaide également en faveur du caractère en place de l'industrie. En effet, la quasi-totalité du matériel se répartit en concentrations clairement distinctes les unes des autres, associant restes de faune et silex taillés. Le développement d'un épisode de pergélisol majeur au sommet de l'unité 5 (grandes fentes en coin) a affecté la distribution verticale des pièces. Sur le terrain, un alignement de certaines pièces était observable au sein des fentes de gel, sans qu'aucun tri granulométrique ne soit prouvé.

Plus de 5000 esquilles ont été recensées dans ce niveau archéologique, dont $69 \%$ mesurent entre 1 et $5 \mathrm{~mm}$. L'ensemble de ces observations géologiques et archéologiques prouve le caractère in situ du matériel lithique et faunique.

\section{2 - LES INDUSTRIES PALÉOLITHIQUES}

La fouille du niveau Hav.2-N0 a permis la découverte de quelques artefacts et restes fauniques en place (fig. 22). La découverte d'occupations saaliennes est suffisamment rare pour être soulignée (Hérisson, 2012 ; Hérisson \& Goval, 2013). Malheureusement, la faible représentativité de l'assemblage ne permet pas de qualifier précisément la nature de ces vestiges.

Le niveau Hav.1-N3 recèle sans doute la découverte la plus atypique. En effet, seuls huit éclats Levallois préférentiels, dont quatre de grandes dimensions ont été mis au jour (fig. 22). Ces éclats sont normalisés, la morphologie et le gabarit de la partie basale ont été recherchés. En effet, les dimensions de ces pièces sont similaires et la moitié d'entre elles sont parfaitement symétriques. Ces éclats ont été produits hors de la zone de fouille et utilisés sur place (ou à proximité immédiate) comme l'attestent l'étude tracéologique et les restes fauniques (Goval et al., 2013). L'interprétation de ce site comme une halte de chasse semble être la plus probable même si la seule présence de pièces tranchantes marquées par de la boucherie laisse le champ possible à d'autres interprétations (aire de dépeçage, de décarnisation). Les observations et les analyses menées sur ces artefacts nous renseignent sur la façon dont ces pièces sont produites, sur leur mobilité et leur utilisation. La découverte du niveau Hav.1-N3 prouve, une fois de plus, la mobilité des Neandertaliens au sein du territoire, et leur degré de planification des activités dans l'espace.

Le niveau Hav.2-N1, bien que sub-contemporain du niveau Hav.1-N3, est différent dans sa composition. Quatre-vingt-deux artefacts ont été récoltés. Le principal objectif de production est l'obtention d'éclats Levallois récurrents et secondairement, préférentiels (fig. 22). 

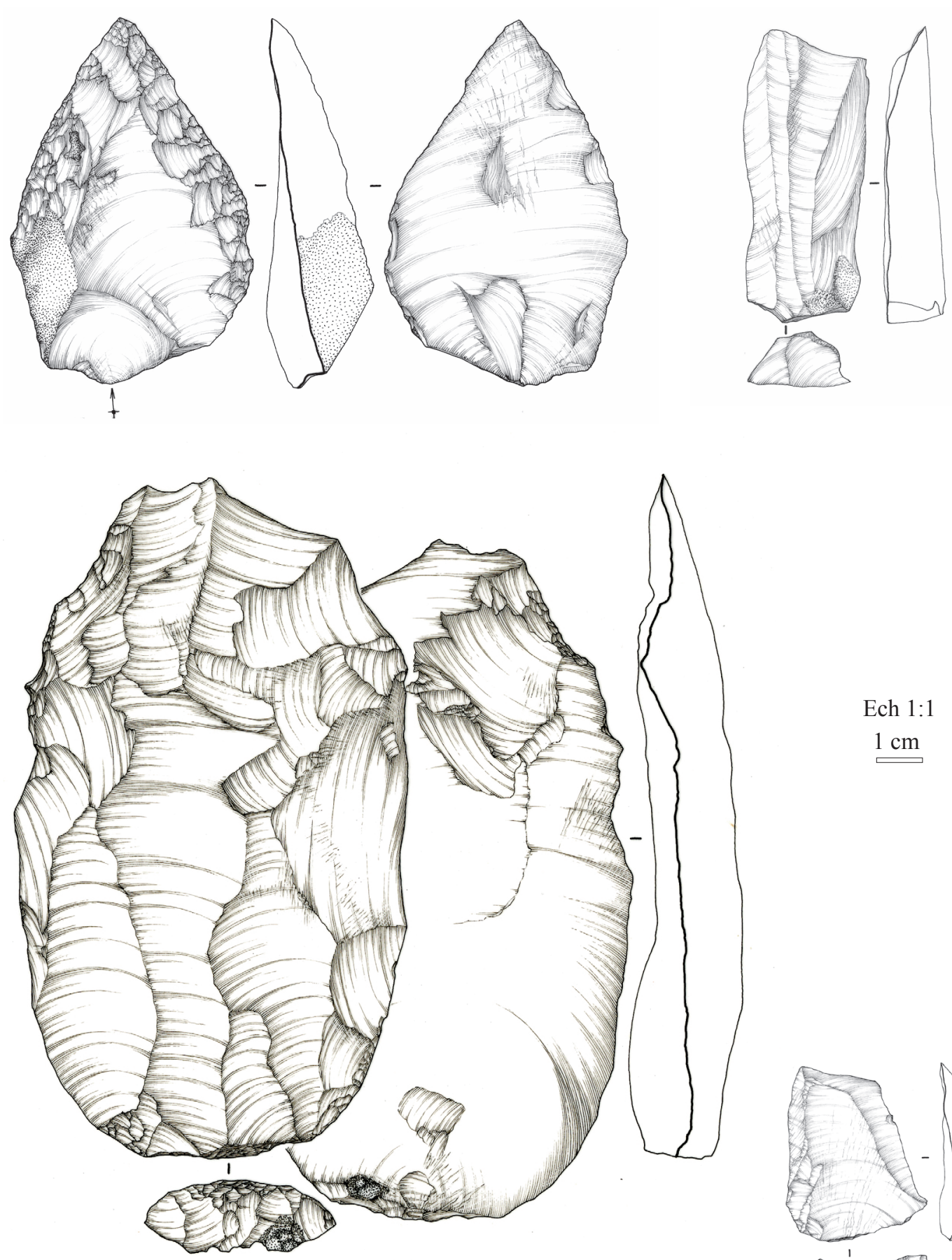

Ech 1:1

$1 \mathrm{~cm}$

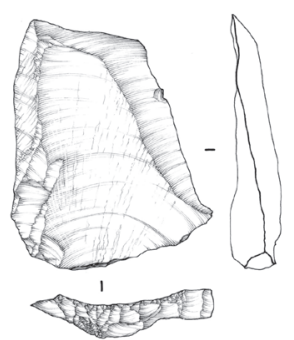

Fig. 22 : Hav.2-N0 : 1/ Pointe Levallois retouchée et éclat laminaire ; Hav.1-N3 : $2 /$ Eclats Levallois préférentiel de grandes dimensions ; Hav.2-N1 : 3/ Eclats Levallois (dessins : E. Boitard-Bidaut)

Fig. 22: Hav.2-N0: 1/Retouched Levallois points and laminar flakes; Hav.1-N3: 2/ Preferential Levallois flakes; Hav.2-N1: 3/Levallois flakes (drawings: E. Boitard-Bidaut)

L'étude tracéologique a mis en évidence la présence de traces d'utilisation sur cinq pièces, malgré des altérations naturelles assez marquées (Goval et al., 2013). Les modes de fonctionnement sont dominés par la coupe de matière tendre à mi-dure, très probablement réalisée dans le cadre d'une activité de boucherie (Goval et al., 2013). Quatorze restes de faune sont associés à ces artefacts. Un fragment de diaphyse d'os long de grand herbivore a également été récolté au sein du niveau Hav.2-N1, il présente les paramètres caractéristiques d'une percussion dynamique sur os frais avec production d'une fracturation de type hélicoïdale. Pour des raisons d'ordre taphonomique, il est certain que l'ensemble des artefacts du niveau Hav.2-N1 ne représente qu'une partie de la production des hommes de passage à Havrincourt. L'analyse des restes dentaires a permis de déterminer la saison estivale comme la période de l'occupation humaine. Le niveau Hav.2-N1 témoigne d'un bref passage des hommes à la saison estivale, dans un paysage dominé par une steppe et un climat continental. L'hypothèse d'une halte où ont été menées des activités de débitage en vue de réaliser des activités de boucherie peut être posée. 
Le niveau Hav.2-N2 reste la découverte la plus inattendue car ce type d'occupation humaine et sa place dans la chronologie régionale sont exceptionnels. La série se compose de 6400 artefacts et 238 restes fauniques répartis en quatre loci. Les hommes ont eu recours à un silex de la craie de mauvaise qualité. L'objectif de production principal est l'obtention de lames (fig. 23). L'ensemble des indices archéologiques qui nous sont parvenus va dans le sens d'une, ou plus probablement, de plusieurs occupations brèves. La mise en commun des données lithiques, fauniques et tracéologiques convergent vers l'idée d'une très courte halte axée vers la récupération de matériaux alimentaires d'origine animale. Bien que la contemporanéité entre les loci ne soit pas avérée, chacun d'entre eux semble fonctionner de manière indépendante mais complémentaire. Ainsi, le locus 1 est plus largement tourné vers la production de supports laminaires, alors que les principales activités de boucheries semblent avoir été réalisées au sein du locus 2. Bien que le nombre minimum d'individus soit peu élevé, le type d'espèce reste varié (principalement du bison, du cheval et du renne). Ce sont principalement des restes de membres antérieurs et postérieurs qui ont été récoltés, peu de restes crâniens ont été dénombrés. Ces observations vont dans le sens d'animaux chassés dans l'environnement immédiat et d'un prélèvement sélectif des différentes parties anatomiques. Par ailleurs, aucune trace de feu (foyers, silex brûlés) n'a été relevée sur le site, aucun outil retouché n'a été confectionné, aucune « arme » n’a été retrouvée.
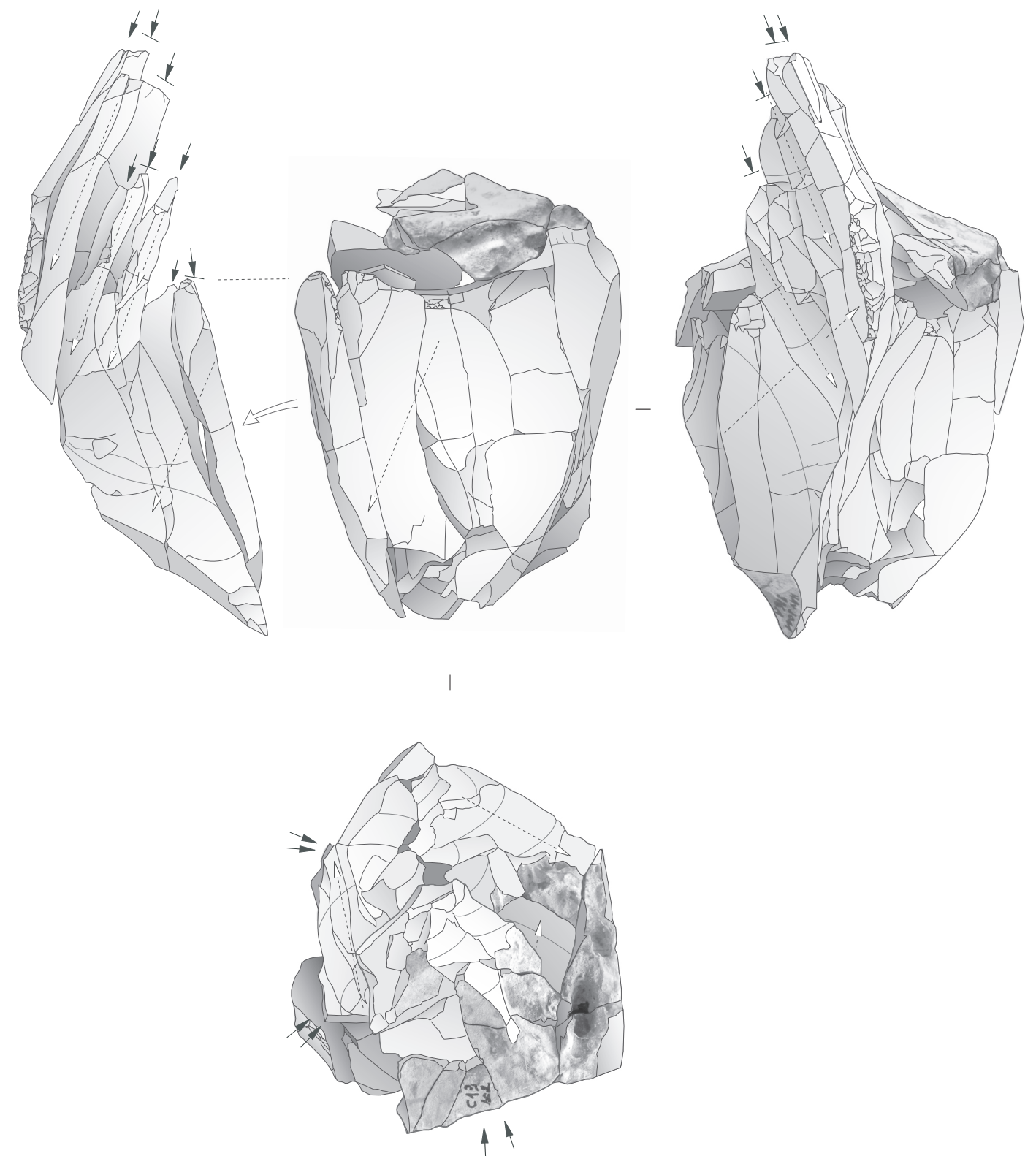

0 5

Fig. 23 : Hav.2-N2 : remontage illustrant la production de supports laminaires (dessin : J. Airvaux).

Fig. 23: Hav.2-N2: refitting showing laminar production (drawing: J. Airvaux). 


\section{3 - SYNTHESE}

Quatre niveaux archéologiques en position primaire et sept assemblages en position secondaire ont été découverts sur l'ensemble des deux secteurs fouillés à Havrincourt. Concernant les niveaux in situ, le niveau le plus ancien est corrélé à l'extrême fin du Saalien (fin SIM 6), deux niveaux sont sub-contemporains et corrélés au début du Pléniglaciaire inférieur $(\sim 65 \mathrm{ka})$ et le niveau supérieur à l'extrême fin du Pléniglaciaire moyen (34-35 ka). De nombreux restes fauniques ont été découverts en association avec les industries lithiques. Malgré des états de conservation variables, l'analyse des cortèges fauniques montre que ceux-ci sont très cohérents. La composition de la faune caractérise bien le spectre des espèces rencontrées couramment en France septentrionale durant les phases froides du Pléistocène supérieur au sein de la steppe à mammouth. Le climat est très continental, $\mathrm{sec}$, avec des alternances saisonnières très marquées. Le paysage est dominé par les espaces très découverts de type steppique, avec une importante biomasse végétale et animale disponible durant la bonne saison. Aucun élément de faune n'indique la présence de zones boisées. C'est donc dans ce contexte que les différentes occupations humaines vont se succéder, à la fin du Paléolithique moyen et durant le début du Paléolithique supérieur à Havrincourt.

La fouille des deux secteurs du gisement d'Havrincourt apporte de nombreuses nouvelles données tant sur le plan de la chronostratigraphie régionale, que des cultures matérielles présentes de la fin du Paléolithique moyen ancien au début du Paléolithique supérieur, ou des types d'occupations dans le territoire. Les découvertes faites à Havrincourt démontrent une fois de plus, l'importance de ce secteur géographique, favorable à la conservation des occupations contemporaines du Pléniglaciaire moyen.

L'intervalle chronologique au sein duquel s'insèrent les occupations d'Havrincourt constitue une période clé dans laquelle s'inscrit progressivement le remplacement de Neandertal par Homo sapiens, deux groupes humains présentant de multiples différences. Les fouilles menées ici permettent de mettre en parallèle l'occupation d'un même territoire dans un environnement assez similaire par deux types humains différents à quelque 25000 ans près.

De par son caractère exceptionnel, une attention toute particulière a été portée au niveau Hav.2-N2. Les datations ${ }^{14} \mathrm{C}$ obtenues sur ce niveau, couplées aux observations chronostratigraphiques, placent le niveau Hav.2-N2 d'Havrincourt dans une frange chronologique s'étendant de la fin de l'Aurignacien au début du Gravettien. Rappelons qu'aucun élément retouché diagnostic n'a été mis en évidence au sein de cette série. Néanmoins, quelques spécificités ont tout de même pu être mises en évidence (percussion tangentielle à la pierre tendre, production bipolaire, recherche de produit laminaire plutôt allongé, initialisation par le biais d'une crête, etc.). Ainsi, bien qu'il faille rester prudent et que les comparaisons régionales restent rares, il semble que la série Hav.2-N2 du gisement d'Havrincourt soit attribuable à du Gravettien ancien. Le gisement de Renancourt 2, fouillé récemment (Paris et al., 2013), a mis en évidence un niveau d'occupation daté du Paléolithique supérieur ancien et comparable en de nombreux points au niveau Hav.2-N2 d'Havrincourt.

D'une manière générale, Havrincourt ne fait pas exception, les loess du Pléniglaciaire supérieur de la France septentrionale sont extrêmement pauvres voire stériles en restes de mammifères. Après plus de 20 ans de recherche et des milliers de sondages profonds il est clair que cette absence n'est pas liée aux conditions de conservation puisque les quelques rares restes retrouvés sont très bien conservés dans les loess calcaires (Le Transloy, Antoine, 1991). De même la malacofaune y est aussi particulièrement peu abondante et diversifiée même au niveau des gleys de toundra associés à des interstades (Moine et al., 2011; Moine, 2014).

Contrairement au Pléniglaciaire moyen, où la présence de grands herbivores est clairement attestée dans plusieurs sites du nord de la France (malgré des conditions de conservations beaucoup moins favorables dues à la décalcification), ces observations indiquent pour le Pléniglaciaire supérieur un milieu froid mais surtout extrêmement découvert et aride où la biomasse est beaucoup trop faible pour subvenir aux besoins de la grande faune mammalienne. Ces données permettent d'expliquer l'absence totale d'occupation archéologique pendant plus de $10 \mathrm{ka}$ au Pléniglaciaire supérieur au niveau régional et plus particulièrement dans l'intervalle 27-16.5 ka (Antoine et al., 2014 ; Moine, 2014).

\section{6 - CONCLUSION}

Cette étude multidisciplinaire des séquences loessiques mises au jour lors des fouilles d'archéologie préventive sur les sites paléolithiques d'Havrincourt en 2010-2011 débouche sur la mise en évidence de données extrêmement riches et cohérentes pour la connaissance du bilan pédosédimentaire du Dernier Glaciaire et du contexte chrono-climatique du Paléolithique dans le nord de la France. Les résultats acquis montrent que la séquence loessique d'Havrincourt dispose de tous les atouts pour devenir une séquence de référence pour le Weichselien du nord de la France.

Les principaux résultats sont les suivants :

-1) mise en évidence d'une nouvelle séquence pédostratigraphique de référence pour le nord de la France, notamment en ce qui concerne le début du Pléniglaciaire inférieur et le Pléniglaciaire moyen, périodes généralement très mal enregistrées dans cette aire géographique (« complexe de sols d'Havrincourt »);

-2) mise en place d'un nouveau canevas géochronologique régional pour le Dernier Glaciaire sur la base des résultats des datations OSL et radiocarbone et confirmation de l'enregistrement dans les environnements loessiques régionaux d'une réponse aux variations climatiques rapides (millénaires); 
-3) révision du schéma pédostratigraphique et chronoclimatique régional en ce qui concerne les périodes des Pléniglaciaires inférieur et moyen $(\sim 65-35 \mathrm{ka})$ et mise en évidence de corrélations avec les séquences du Dernier Glaciaire en Europe du Nord-Ouest (Belgique et Allemagne);

-4) mise en évidence d'un environnement typiquement steppique particulièrement favorable à l'expansion des faunes de grands mammifères et de rongeurs dans la première moitié du Pléniglaciaire moyen entre $\sim 60-58$ et $45 \mathrm{ka}$, dans un milieu dominé par la pédogenèse (unité 7). Par contre, la partie supérieure du complexe d'Havrincourt (unité 6), qui contient le niveau du Paléolithique supérieur ancien, montre des assemblages de milieu plus froid et plus ouvert avec le Renne et le Rhinocéros laineux. Enfin, comme dans tous les autres sites du nord de la France, la biomasse semble totalement insignifiante pendant la mise en place des loess du Pléniglaciaire supérieur;

- 5) mise en évidence d'une succession unique d'horizons périglaciaires : cinq réseaux de fentes associées à des gleys de toundra, dont deux dégradés associés à des structures de fusion (chenaux de fonte / thermokarst SF-1 et SF-2). Cette succession, datée par OSL et ${ }^{14} \mathrm{C}$, constitue une première séquence régionale de référence qui, en ce qui concerne les niveaux principaux, montre de fortes analogies avec celles des régions loessiques limitrophes comme la Normandie, le nord du Bassin Parisien ou la Belgique;

6) découverte de quatre niveaux d'occupation humaine en place dont un niveau archéologique exceptionnel attribuable au Paléolithique supérieur ancien (Gravettien ancien). La bonne conservation des niveaux archéologiques (versant en pente douce favorisant le piégeage des loess et l'enfouissement rapide des artefacts), associée à l'analyse de la séquence pédosédimentaires et aux datations permet de proposer un calage chronostratigraphique et chronoclimatique précis pour les niveaux paléolithiques fouillés.

\section{RÉFÉRENCES BIBLIOGRAPHIQUES}

ADAM W., 1960 - Mollusques. Tome 1 : Mollusques terrestres et dulcicoles. Institut royal des Sciences naturelles de Belgique, Bruxelles, $402 \mathrm{p}$.

AITKEN M.J., 1985 - Thermoluminescence Dating. Academic Press, London, $359 \mathrm{p}$.

ANDERSON R., 2005 - An annotated list of the non-marine Mollusca of Britain and Ireland. Journal of Conchology, 38 (6), 607-637.

ANTOINE P., 1988 - Contribution à l'étude des loess du Pléistocène supérieur du bassin de la Somme. Revue Archéologique de Picardie, 1-2, 25-44.

ANTOINE P., 1990 - Chronostratigraphie et environnement du Paléolithique du bassin de la Somme. Publications du Centre d'Études et de Recherches Préhistoriques, 2, 1-231.

ANTOINE P., 1991 - Nouvelles données sur la stratigraphie du Pléistocène supérieur de la France septentrionale, d'après les sondages effectués sur le tracé du TGV Nord. Publications du Centre d'Études et de Recherches Préhistoriques, 3, 9-20.

ANTOINE P., 2002 - Les loess en France et dans le nord-ouest européen. Revue Française de Géotechnique, 99, 3-21.

ANTOINE P., MUNAUT A.-V., \& SOMMÉ J., 1994 - Réponse des environnements à l'évolution climatique du Début Glaciaire weich- sélien : données de la France du Nord Ouest. Quaternaire, 5 (3-4), 151-156.

ANTOINE P., LAUTRIDOU J.-P., SOMME J., AUGUSTE P., AUFFRET J.-P., BAIZE S., CLET-PELLERIN M., COUTARD J.-P., DEWOLF Y., DUGUÉ O., JOLY F., LAIGNEL B., LAURENT M., LAVOLLÉ M., LEBRET P., LEFEBVRE D., LÉCOLLE F., LIMONDIN-LOZOUET N., MUNAUT A.-V., OZOUF J.-C., QUESNEL F. \& ROUSSEAU D.-D., 1998 - Les formations quaternaires de la France du Nord-Ouest : limites et corrélations. Quaternaire, 9 (3), 227-241.

ANTOINE P., ROUSSEAU D.-D., LAUTRIDOU J.-P. \& HATTÉ C., 1999 - Last interglacial-glacial climatic cycle in loess-paleosol successions of north-western France. Boreas, 28 (4), 551-563.

ANTOINE P., ROUSSEAU D.-D., ZÖLLER L., LANG A., MUNAUT A.-V., HATTÉ C. \& FONTUGNE M., 2001 - Highresolution record of the last Interglacial-glacial cycle in the Nussloch loess-palaeosol sequences, Upper Rhine Area Germany. Quaternary International, 76/77, 211-229.

ANTOINE P., FRECHEN M., LOCHT J.-L., DEPAEPE P., MUNAUT A.-V., ROUSSEAU D.-D. \& SOMMÉ J., 2002a - Eemian and Weichselian early glacial pedosedimentary records in northern France: the background of middle palaeolithic occupations during OIS 5 and early OIS 4. Publications du Centre d'Etudes et de Recherches Préhistoriques, 8, 75-88.

ANTOINE P., ROUSSEAU D.-D., HATTÉ C., ZÖLLER L., LANG A., FONTUGNE M. \& MOINE O., 2002b - Evénements éoliens rapides en contexte loessique: l'exemple de la séquence du pléniglaciaire supérieur weichsélien de Nussloch (vallée du Rhin, Allemagne). Quaternaire, 13 (3-4), 199-208.

ANTOINE P., AUGUSTE P., BAHAIN J.-J., COUDRET P., DEPAEPE P., FAGNART J.-P., FALGUÈRES N., FONTUGNE M., FRECHEN M., HATTÉ C., LAMOTTE A., LAURENT M., LIMONDIN-LOZOUET N., LOCHT J.-L., MERCIER N., MOIGNE A.-M., MUNAUT A.-V., PONEL P. \& ROUSSEAU D.-D., 2003a - Paléoenvironnements pléistocènes et peuplements paléolithiques dans le bassin de la Somme (nord de la France). Bulletin de la Société Préhistorique Française, 100 (1), 5-28.

ANTOINE P., BAHAIN J.-J., DEBENHAM N., FRECHEN M., GAUTHIER A., HATTÉ C., LIMONDIN-LOZOUET N., LOCHT J.-L., RAYMOND P. \& ROUSSEAU D.-D., 2003b - Nouvelles données sur le Pléistocène du nord du Bassin Parisien : les séquences loessiques de Villiers-Adam (Val d'Oise, France). Quaternaire, 14 (4), 219-235.

ANTOINE P., CATT J., LAUTRIDOU J.-P. \& SOMMÉ J., 2003c - The loess and coversands of northern France and southern England. Journal of Quaternary Science, 18 (3-4), 309-318.

ANTOINE P., MARCHIOL A., BROCANDEL M. \& GROS Y., 2005 - Découverte de structures périglaciaires (sand-wedges et composite-wedges) sur le site de stockage de déchets radioactifs de l'Aube (France). Comptes Rendus Géosciences, 337 (16), 1462-1473.

ANTOINE P., ROUSSEAU D.-D., MOINE O., KUNESCH S., HATTÉ C., LANG A., TISSOUX H. \& ZÖLLER L., 2009 - Rapid and cyclic aeolian deposition during the Last Glacial in European loess: a high-resolution record from Nussloch, Germany. Quaternary Science Reviews, 28 (25-26), 2955-2973.

ANTOINE P., MOINE O. \& HATTÉ C., 2013 - Les processus thermokarstiques : marqueurs d'épisodes de réchauffement climatique rapides au cours du Dernier Glaciaire dans les séquences loessiques ouest-européennes / Thermokarst processes: evidences for rapid climatic warming events during the Last Glacial in west-European loess series. In French Arctic Initiative's inaugural symposium "Arctic: the major scientific issues", Collège de France, Paris, 3-5 juin 2013. http://www.chantierarctique.fr/en/uploads/Permafrost_4_Antoine.pdf

ANTOINE P., LOCHT J.-L., LIMONDIN-LOZOUET N., AUGUSTE $P$, BAHAIN $J .-J$, FAGNART J.-P., DEBENHAM N. \& DUCROCQ T., 2014 - Quaternaire et géoarchéologie de la Préhistoire : le modèle de la vallée Somme (France) et des régions avoisinantes. In N. Carcaud G. \& Arnaud-Fassetta (eds.), La géoarchéologie française au XXIe siècle. CNRS Éditions, Paris.

AUGUSTE P., 2009 - Evolution des peuplements mammaliens en Europe du Nord-Ouest durant le Pléistocène moyen et supérieur. Le cas de la France septentrionale. Quaternaire, 20 (4), 527-550.

AUGUSTE P., 2012 - L'homme et l'animal au Pléistocène en France septentrionale. Un quart de siècle de recherches paléontologiques et archéozoologiques dans le nord de la France. Mémoire d'Habilitation à Diriger des Recherches, Université Lille 1, Villeneuve-d'Ascq, 2 vol., 251 p.

BANK R. A., FALKNER, G. \& VON PROSCHWITZ T., 2001 - CLECOM-PROJECT. A revised checklist of the non-marine Mollusca of Britain and Ireland. Heldia, 5 (3), 41-72. 
BLACK R.F., 1976 - Periglacial features indicative of permafrost: Ice and soils wedges. Quaternary Research, 6 (1), 3-26.

BOND G., BROECKER W., JOHNSEN S., MCMANUS J., LABEYRIE L., JOUZEL J. \& BONANI G., 1993 - Correlations between climate records from North Atlantic sediments and Greenland ice. Nature, $\mathbf{3 6 5}$ (6442), 143-147.

BØTTER-JENSEN L., BULUR E., MURRAY A.S. \& POOLTON N.R.J. 2002 - Enhancements in luminescence measurement techniques. Radiation Protection Dosimetry, 101 (1-4), 119-124.

COGNÉ, J.-P., 2003 - PaleoMac: A Macintosh TM application for treating paleomagnetic data and making plate reconstructions. Geochemistry, Geophysics, Geosystems, 4 (1), 8 p., 1007, doi 10.1029/2001GC000227 (2003).

DESCHODT L., BARBIER P., DJEMMALI N., DRWILA G. FERAY P, TEHEUX E., BECU B. \& AUGUSTE $P$, 1998 - Onnaing, Usine Toyota, Rapport des sondages archéologiques profonds. 06/98 Villeneuve d'Ascq. Institut National Archéologiques préventives, Rapport de sondage, Direction Régionale des Affaires Culturelles du Nord-Pas-de-Calais, Service Régional de l'Archéologie, $66 \mathrm{p}$.

FAIRBANKS R.G., MORTLOCK R.A., CHIU T.-C., CAO L. KAPLAN A., GUILDERSON T.P., FAIRBANKS T.W., BLOOM A.L., GROOTES P.M. \& NADEAU M.-J., 2005 - Radiocarbon calibration curve spanning 0 to 50,000 years BP based on paired ${ }^{230} \mathrm{Th} /{ }^{234} \mathrm{U} /{ }^{238} \mathrm{U}$ and ${ }^{14} \mathrm{C}$ dates on pristine corals. Quaternary Science Reviews, 24 (16-17), 1781-1796.

FALKNER G., RIPKEN T.E.J. \& FALKNER M., 2002 - Mollusques continentaux de France. Liste de Référence annotée et Bibliographie. Patrimoines naturels, 52, 1-350.

FRENCH H.M., 2007 - The Periglacial Environment, third edition John Wiley \& Sons, Chichester, $458 \mathrm{p}$.

FRENCH H. \& SHUR Y. 2010 - The principles of cryostratigraphy. Earth-Science Reviews, 101 (3-4), 190-206.

FRECHEN M., SCHWEITZER U. \& ZANDER A., 1996 - Improvements in sample preparation for the fine grain technique. Ancient $T L, 14$ (2), 15-17.

FRECHEN M, VAN VLIET-LANOË B. \& VAN DEN HAUTE P., 2001 - The Upper Pleistocene loess record at Harmignies/Belgium, high resolution terrestrial archive of climate forcing. Palaeogeography, Palaeoclimatology, Palaeoecology, 173 (3-4), 175-195.

FRECHEN M., OCHES E.A. \& KOHFELD K.E., 2003 - Loess in Europe-mass accumulation rates during the Last Glacial Period. Quaternary Science Reviews, 22 (18-19), 1835-1875.

GERMAIN L., 1930-1931 - Mollusques terrestres et fluviatiles. Faune de France, 21-22. Librairie de la Faculté des Sciences, Paris, 2 vol., $893 \mathrm{p}$

GOVAL É., 2012 - Peuplements néandertaliens dans le nord de la France: territoires, industries lithiques et occupations humaines durant la phase récente du Paléolithique moven. Recherches Archéologiques, 4. CNRS Éditions et INRAP, Paris, $312 \mathrm{p}$.

GOVAL É. \& HÉRISSON D., 2006 - Coexistence des chaînes opératoires Levallois et laminaires au sein des assemblages $\mathrm{C} 12$ et $\mathrm{C}$ de Riencourt-lès-Bapaume (Pas-de-Calais, France. Notae Praehistoricae, 26, 25-39.

GOVAL É. \& LOCHT J.-L., 2009 - Remontages, systèmes techniques et répartitions spatiales dans l'analyse du site weichselien ancien de Fresnoy-au-Val (Somme, France). Bulletin de la Société Préhistorique Française, 106 (4), 653-678

GOVAL É. avec la collaboration de ANTOINE P., AUGUSTE P. BAHAIN J.-J., CLAUD E., COUTARD S., FONT C., GUERRIN G.,HÉRISSON D., HULIN G.,JAMET G., MOINE O.,PRILAUX G., SCHMIDT E. \& VALLADAS H., 2013 - Des derniers Néandertaliens aux premiers Homo Sapiens : les occupations paléolithiques d'Havrincourt "Les Bosquets » (Pas-de-Calais). Rapport de fouille, Canal Seine-Nord Europe, fouille 22, Nord-Pas-de-Calais, Pas-deCalais, Havrincourt, Institut National de Recherches Archéologiques Préventives, Croix-Moligneaux, $432 \mathrm{p}$

GUADELLI J.-L., 1998 - Détermination de l'âge des chevaux fossiles et établissement des classes d'âge. Paléo, 10, 87-93.

GUÉRIN G., MERCIER N.,ADAMIEC G., 2011 - Dose-rate conversion factors: update. Ancient TL, 29 (1), 5-8.

GUÉRIN G. \& VALLADAS H., 2014 - Cross-calibration between beta and gamma sources using quartz OSL: Consequences of the use of the SAR protocol in optical dating. Radiation Measurements, 68, 31-37.

HAESAERTS P. \& VAN VLIET B., 1973 - Evolution d'un permafrost fossile dans les limons du dernier glaciaire à Harmignies (Belgique). Bulletin de l'Association Française pour l'Étude du Quaternaire, 10 (3), 151-164.

HAESAERTS P., JUVIGNÉ É, KUYL O, MÜCHER H. \& ROEBROEKS W., 1981 - Compte rendu de l'excursion du 13 Juin
1981, en Hesbaye et au Limbourg Néerlandais, consacrée à la chronostratigraphie des loess du Pléistocène supérieur. Annales de la Société Géologique de Belgique, 104, 223-240.

HAESAERTS P., MESTDAGH H. \& BOSQUET D. 1999 - The sequence of Remicourt (Hesbaye, Belgium): new insights on the pedo- and chronostratigraphy of the Rocourt soil. Geologica Belgica, $2(3-4), 5-27$.

HÉRISSON D., 2012 - Etude des comportements des premiers Néandertaliens du Nord de la France: les occupations saaliennes des gisements de Biache-Saint-Vaast et de Therdonne. Thèse de Doctorat, Université de Lille 1, Villeneuve-d'Ascq, 502 p.

HÉRISSON D. \& GOVAL É., 2013 - Du Paléolithique inférieur au début du Paléolithique supérieur dans le Nord de la France : lumière sur les premières découvertes du Canal Seine-Nord Europe. Notae Praehistoricae, 33, 91-104.

HEXT G.R., 1963 - The estimation of second-order tensors, with related tests and designs. Biometrika, 50 (3-4), 353-373.

JAMET G., 2011 - La séquence loessique d'Havrincourt (Pas-deCalais): Approche analytique d'un enregistrement pédosédimentaire du dernier cycle climatique interglaciaire-glaciaire, Mémoire de Master 2, Université de Bourgogne, Dijon, $81 \mathrm{p}$.

JELÍNEK V., 1976 - The statistical theory of measuring anisotropy of magnetic susceptibility of rocks and its application. Geophyzika, Brno, $88 \mathrm{p}$.

JELÍNEK V., 1981 - Characterization of the magnetic fabric of rocks. Tectonophysics, 79 (3-4), 63-67.

JUVIGNÉ É., TALLIER É., HAESAERTS P. \& PIRSON S., 2008 - Un nouveau stratotype du téphra de Rocourt dans la carrière de Romont (Eben/ Bassenge, Belgique). Quaternaire, 19 (2), 133-139.

KADEREIT A., KIND C.-J. \& WAGNER G.A., 2013 - The chronological position of the Lohne Soil in the Nussloch loess section - re-evaluation for a European loess-marker horizon. Quaternary Science Reviews, 59, 67-86.

KERNEY M.P. CAMERON R.A.D. \& JUNGBLUTH J.H. 1983 - Die Landschnecken Nord- und Mitteleuropas, Verlag Paul Parey, Hamburg \& Berlin, $384 \mathrm{p}$.

LAGROIX F. \& BANERJEE S.K., 2002 - Paleowind directions from the magnetic fabric of loess profiles in central Alaska. Earth and Planetary Science Letters, 195 (1-2), 99-112.

LAGROIX F. \& BANERJEE S.K., 2004 - The regional and temporal significance of primary aeolian magnetic fabrics preserved in Alaskan loess. Earth and Planetary Science Letters, 225 (3-4), 379-385

LAUTRIDOU J.-P., 1987. Le cycle périglaciaire pléistocène en Europe du Nord-Ouest et plus particulièrement en Normandie. Thèse de Doctorat d'État, Université de Caen, Caen. Centre de Géomorphologie du CNRS, Caen, 2 vol., 908 p.

LOCHT J.-L. (dir.), ANTOINE P., AUGUSTE P., CASPAR P., DEPAEPE P., ENGELMANN A., FRECHEN M., MICHEL V., MUNAUT A.-V., RÉVILLION S. \& SWINNEN C., 2002 - Bettencourt-Saint-Ouen (Somme) : Cinq occupations paléolithiques au début de la dernière glaciation. Documents d'Archéologie Francaise, 90. Éditions de la Maison des Sciences de l'Homme, Paris, $169 \mathrm{p}$.

LOCHT J.-L., ANTOINE P., BAHAIN J.-J., DWRILA G., RAYMOND P., LIMONDIN-LOZOUET N., GAUTHIER A, DEBENHAM N., FRECHEN M., ROUSSEAU D.-D., HATTÉ C., HAESAERTS P. \& METSDAGH H., 2003 - Le gisement paléolithique moyen et les séquences pléistocènes de Villiers-Adam (Val d'Oise, France) : Chronostratigraphie, environnement et implantations humaines. Gallia Préhistoire, 45, 1-111.

LOCHT J.-L., ANTOINE P., AUGUSTE P., BAHAIN J.-J., DEBENHAM N., FALGUÈRES C., FARKH S. \& TISSOUX H., 2006 - La séquence loessique pléistocène supérieur de Savy (Aisne, France) : stratigraphie, datations et occupations paléolithiques. Quaternaire, 17 (3), 269-275

LOŽEK V., 1964 - Quartärmollusken der Tschechoslowakei. Rozpravy Ústredního Ústuvu Geologického, 31, 1-374.

MALLYE J.-B. \& GUÉRIN C., 2002 - Le gisement pléistocène supérieur de la grotte de Jaurens à Nespouls, Corrèze, France : les Mustelidae (Mammalia, Carnivora). Cahiers Scientifiques - Centre de Conservation et d'Étude des Collections, 4, 15-53.

MARCOTT S.A., CLARK P.U., PADMAN L., KLINKHAMMER G.P., SPRINGER S.R., LIU Z., OTTO-BLIESNER B.L., CARLSON A.E., UNGERER A., PADMAN J., HE F., CHENG J. \& SCHMITTNER A., 2011 - Ice-shelf collapse from subsurface warming as a trigger for Heinrich events. Proceedings of the National Academy of Sciences of the United States of America, 108 (33), 13415-13419. 
MARCY T., SELLIER N., DEVRED V. \& DUBOIS S., 2009 - Bourlon, Havrincourt, Graincourt-lès-Havrincourt, Sainslès-Marquions (Pas-de-Calais). Rapport de diagnostic de la ZD7, Canal Seine-Nord Europe, Institut National de Recherches Archéologiques Préventives, Croix-Moligneaux, $116 \mathrm{p}$

MATSUOKA N., 2011 - Climate and material controls on periglacial soil processes: Toward improving periglacial climate indicators. Quaternary Research, 75 (2), 356-365.

MOINE O., 2008 - West-european malacofauna from loess deposits of the Weichselian Upper Pleniglacial: compilation and preliminary analysis of the database. Quaternaire, 19 (1), 11-29.

MOINE O., 2011 - Rapport sur la malacofaune des loess du Pléniglaciaire weichsélien moyen et supérieur de Languevoisin (80). Institut National de Recherches Archéologiques Préventives NordPicardie, Amiens, $8 \mathrm{p}$.

MOINE O., 2014 - Weichselian Upper Pleniglacial environmental variability in north-western Europe reconstructed from terrestrial mollusc faunas and its relationship with the presence/absence of human settlements, Ouaternary international, 337, 90-113.

MOINE O., ROUSSEAU D.-D. \& ANTOINE P., 2005 - Terrestrial molluscan records of Weichselian Lower to Middle Pleniglacial climatic changes from the Nussloch loess series (Rhine Valley, Germany): the impact of local factors. Boreas, 34 (3), 363-380.

MOINE O., ROUSSEAU D.-D. \& ANTOINE $\mathbf{P}, 2008$ - The impact of Dansgaard-Oeschger cycles on the loessic environment and malacofauna of Nussloch (Germany) during the Upper Weichselian. Ouaternary Research, 70 (1), 91-104.

MOINE O., ANTOINE P., DESCHODT L. \& SELLIER-SEGARD N., 2011 - Enregistrements malacologiques à haute résolution dans les loess et les gleys de toundra du Pléniglaciaire weichselien supérieur : premiers exemples du nord de la France. Quaternaire, 22 (4), 307-325.

MOURER-CHAUVIRÉ C., 1980 - Le gisement Pléistocène supérieur de la grotte de Jaurens à Nespouls (Corrèze, France) : les Equidés (Mammalia, Perissodactyla). Nouvelles Archives du Muséum d'Histoire Naturelle de Lyon, 18, 17-60.

MURRAY A.S. \& WINTLE A.G., 2003 - The single aliquot regenerative dose protocol: potential for improvements in reliability. Radiation Measurements, 37 (4-5), 377-381.

MURTON J.B., 2007 - Periglacial landforms | Ice wedges and Ice Wedge Casts. In S.A. Elias (ed.), Encyclopedia of Quaternary Science. Elsevier, Amsterdam, 2153-2170.

PARIS C., FAGNART J.-P. \& COUDRET P., 2013 - Du Gravettien final dans le Nord de la France ? Nouvelles données à AmiensRenancourt (Somme, France). Bulletin de la Société Préhistorique Française, 110 (1), 123-126.

PISSART A., 1987 - Géomorphologie Périglaciaire : textes des leçons de la Chaire Francqui belge. Laboratoire de Géomorphologie et Géologie du Quaternaire de l'Université de Liège, Liège, 135 p.

PRESCOTT J.R. \& STEPHAN L.G. 1982 - The contribution of cosmic radiation to the environmental dose for thermoluminescent dating: Latitude, altitude and depth dependencies, PACT (Strasbourg), 6, 17-25.

PRESCOTT J.R. \& HUTTON J.T., 1994 - Cosmic ray contribution to dose rates for luminescence and ESR dating: large depths and longterm time variations. Radiation Measurements, 23 (2-3), 497-500.

PUISSÉGUR J.-J., 1978 - Les mollusques des séries loessiques à Achenheim. Recherches Géographiques à Strasbourg, 7, 71-96.

REES-JONES J. \& TITE M.-S., 1997 - Optical dating results for British archaeological sediments. Archaeometry, 39 (1), 177-187.

RÉVILLION S. \& TUFFREAU A. (dir.), 1998 - Les industries laminaires au Paléolithique moyen : actes de la table ronde internatio- nale organisée par l'ERA 37 du CRA-CNRS à Villeneuve-d'Ascq, 13 et 14 novembre 1991. Dossier de documentation archéologique, 18. CNRS Éditions, Paris, 193 p.

REIMER P.J., BARD E., BAYLISS A., BECK J.W., BLACKWELL P.G., BRONK RAMSEY C., BUCK C.E., CHENG H., EDWARDS R.L., FRIEDRICH M., GROOTES P.M., GUILDERSON T.P., HAFLIDASON H., HAJDAS I., HATTE C., HEATON T.J., HOFFMANN D.L., HOGG A.G., HUGHEN K.A., KAISER K.F., KROMER B., MANNING S.W., NIU M., REIMER R.W., RICHARDS D.A., SCOTT E.M., SOUTHON J.R, STAFF R.A, TURNEY C.S.M. \& VAN DER PLICHT J., 2013 - Intcal13 and Marine13 radiocarbon age calibration curves 0-50,000 years cal BP. Radiocarbon, 55 (4), 1869-1887.

ROCROI J.-P., BICHAIN J.-M. \& GARGOMINY O., 2007 - De Trichia à Trochulus (Gastropoda, Hygromiidae). MalaCo, 4, 150.

SCHIERMEYER J., 2002 - Würmzeitliche Lößmollusken aus der Eifel. Doktorarbeit, Universität Düsseldorf, Düsseldorf, 283 p.

SELLIER N., 2005 - Bapaume, le Petit Moulin. Rapport de diagnostic, Institut National de Recherches Archéologiques Préventives NordPicardie, Amiens, $15 \mathrm{p}$.

SIMA A., KAGEYAMA M., ROUSSEAU D.-D., RAMSTEIN G., BALKANSKI Y., ANTOINE P. \& HATTÉ C. 2013 - Modeling dust emission response to North Atlantic millennial-scale climate variations from the perspective of East European MIS 3 loess deposits. Climate of the Past, 9, 1385-1402.

SOMMÉ J., 1975 - Les plaines du Nord de la France et leur bordure : étude géomorphologique. Thèse de Doctorat d'État, Université Paris 1 Panthéon-Sorbonne, Paris, 3 vol., 790 p.

SOMMÉ J., PAEPE R. \& LAUTRIDOU J.-P., 1980 - Principes, méthodes et système de la stratigraphie du Quaternaire dans le nordouest de la France et la Belgique. In J. Chaline (dir.), Problèmes de stratigraphie quaternaire en France et dans les pays limitrophes. Supplément au Bulletin de l'Association Française pour l'Étude du Quaternaire N.S., 1. Association Française pour 1'Étude du Quaternaire, Paris, 148-162.

VALLI A. \& GUÉRIN C. 2000 - Le gisement pléistocène supérieur de la grotte de Jaurens à Nespouls, Corrèze, France : les Cervidae (Mammalia, Artiodactyla). Cahiers Scientifiques - Centre de Conservation et d'Étude des Collections, 1, 41-87.

VALLIN L. \& MASSON B., 2003 - Le gisement moustérien d'Hermies - Le Tio Marché. Rapport de fouille programmée, campagne 2002, Service Régional d'Archéologie Nord-Pas-de-Calais, Lille, $34 \mathrm{p}$.

VAN VLIET-LANOË B., 1987 - Dynamique périglaciaire actuelle et passée : apport de l'étude micromorphologique et de l'expérimentation. Bulletin de l'Association Française pour l'Étude du Quaternaire, 24 (3), 113-132.

VAN VLIET-LANOË B., 1990 - Le pédocomplexe de Warneton : où en est-on ? Bilan paléopédologique et micromorphologique. Quaternaire, 1 (1), 65-76.

VAN VLIET-LANOË B., FAGNART J.-P., LANGHOR R. \& MUNAUT A.-V., 1992 - Importance de la succession des phases écologiques anciennes et actuelles dans la différenciation des sols lessivés de la couverture loessique d'Europe occidentale : argumentations stratigraphique et archéologique. Sciences du Sol, 30 (2), 75-93.

WANG X.L., WINTLE A.G., DU J.H., KANG S.G. \& LU Y.C., 2011 - Recovering laboratory doses using fine-grained quartz from Chinese loess. Radiation Measurements, 46 (10), 1073-1081.

ZILCH A. \& JAECKEL S.G.A., 1962 - Mollusken. Die Tierwelt Mitteleuropas, 2 (1). Quelle \& Meyer, Leipzig, 294 p. 